This is re-typesetting of the book first published as

PUBLICATIONS OF THE MATHEMATICAL SOCIETY OF JAPAN

13

\title{
TWO APPLICATIONS OF LOGIC TO MATHEMATICS
}

\author{
by \\ Gaisi Takeuti
}

\begin{abstract}
Kanô Memorial Lectures 3
Iwanami Shoten, Publishers

and

Princeton University Press
\end{abstract}

1978

The present work was typeset by $\mathcal{A} \mathcal{M}$ - ${ }^{A} T_{E} X$, the $T_{E} X$ macro systems of the American Mathematical Society.

$T_{E} X$ is the trademark of the American Mathematical Society.

(C)2012 by the Mathematical Society of Japan.

All rights reserved. The Mathematical Society of Japan retains the copyright of the present work. No part of this work may be reproduced, stored in a retrieval system, or transmitted, in any form or by any means, electronic, mechanical, photocopying, recording or otherwise, without the prior permission of the copyright owner. 


\section{TWO APPLICATIONS OF LOGIC TO MATHEMATICS}

by Gaisi Takeuti 
Preface

This book consists of two independent parts, each of which has its own list of references.

Part I is Boolean valued analysis. Most of the content of this part was discussed in my courses in the spring semester of 1974 at the University of Illinois and the summer semester of 1975 at the Universität Heidelberg.

I appreciate the interest shown by my colleagues and I have profited from discussions with them and from information and advice that they have provided. In particular I want to recognize the assistance of Joseph Doob, Peter Loeb, Heinrich Lotz, Earl Berkson and especially David Berg for his instructive discussion of Hilbert spaces.

Part II is titled "A conservative extension of Peano Arithmetic". The content is a revision of my lecture notes for a course offered in the fall semester of 1972 at the University of Illinois. The revision was used in a course that I offered in the summer semester of 1975 at the Universität Heidelberg. I started this work in the course of correspondence with Georg Kreisel. I have profited from his correspondence. The original lecture notes were prepared with the assistance of Mariko Yasugi, and editorial assistance was provided by my colleague Wilson Zaring. Mr. Mamoru Kurata and Mr. Susumu Hayasi have read the proofs of Part I and Part II respectively.

This book was written at the suggestion of my teacher Professor S. Iyanaga. I also received encouragement from Professor Gödel and I express my deep appreciation to him and to all who contributed.

Urbana

September, 1976

Gaisi Takeuti 


\section{Contents}

$\begin{array}{ll}\text { Preface } & \text { ii }\end{array}$

$\begin{array}{ll}\text { Introduction } & 1\end{array}$

I Boolean Valued Analysis 3

1 Boolean Valued Analysis Using Projection Algebras 7

1.1 Hilbert space . . . . . . . . . . . . . . . . . . 7

1.2 The model $V^{(\mathcal{B})} \ldots \ldots \ldots \ldots \ldots \ldots$

1.3 Real numbers in $V^{(\mathcal{B})} \ldots \ldots \ldots \ldots \ldots \ldots \ldots$

1.4 The interpretation of elementary theorems . . . . . . . . . 21

1.5 Miscellaneous interpretations . . . . . . . . . . . . . . 28

1.6 Convergence . . . . . . . . . . . . . . . . . . . . . . . . . 29

1.7 Semi-group of self-adjoint operators _ . . . . . . . . . . 32

1.8 Complete Boolean algebras of Banach spaces . . . . . . . . . 36

1.9 Piecewise convergence . . . . . . . . . . . . . . . 37

1.10 Simultaneous spectrums $\ldots \ldots \ldots \ldots$

1.11 Quantum Logic . . . . . . . . . . . . . . . . . . . . 42

2 Boolean Valued Analysis Using Measure Algebras 47

2.1 Measure algebras . . . . . . . . . . . . . . . . . . . 47

2.2 Real numbers in the model . . . . . . . . . . . . . . . . 48

2.3 Continuous functions in the model . . . . . . . . . . . . . . 52

2.4 Baire functions and Borel sets in the model . . . . . . . . . 55

2.5 Integration and differentiation in the model . . . . . . . . . 58

2.6 Relation between projection algebras and measure algebras . . . 62

\section{A Conservative Extension of Peano Arithmetic 67}

3 Real Analysis $\quad 73$

3.1 The first system . . . . . . . . . . . . . . . . 73

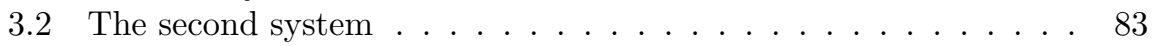

3.3 Defining reals . . . . . . . . . . . . . . . . . 85 
3.4 Functions . . . . . . . . . . . . . . . . . . . . 87

3.5 Sequences . . . . . . . . . . . . . . . . . . 89

3.6 Continuous functions . . . . . . . . . . . . . . . . . . . 91

3.7 Differentiation ...................... 93

3.8 Integration . . . . . . . . . . . . . . . . . . 95

3.9 Sequences of functions . . . . . . . . . . . . . . . . . 97

3.10 Infinite series and series of functions . . . . . . . . . . . . . . . 98

3.11 Higher derivatives . . . . . . . . . . . . . . . . . . . . . 101

3.12 Functions of several variables . . . . . . . . . . . . . . 102

4 Complex Analysis 105

4.1 A system of complex numbers . . . . . . . . . . . . . . . . . 105

4.2 Analytic functions . . . . . . . . . . . . . . . . . . . 109

4.3 Integration . . . . . . . . . . . . . . . . . . . . 112

4.4 Cauchy's Theorem for nice regions . . . . . . . . . . . . 115

4.5 Cauchy's Integral Formula . . . . . . . . . . . . . . . . . . . . . 116

4.6 The general form of Cauchy's Theorem . . . . . . . . . . . . . . . 120

4.7 Miscellaneous remarks . . . . . . . . . . . . . . . . . . . . 121

4.8 Final remarks . . . . . . . . . . . . . . . . . . 122 
Introduction

Mathematical logic is reflection on mathematics. More specifically it is reflection on such questions as, What is logical validity? What is effective calculability? What is a set? What are the basic principles of the universe of sets. Apart from reflecting on these questions themselves, and proving relevant metatheorems, one also wants to know the chances of an effective use of various logical metatheorems in specific branches of mathematics. For example, one might say that Abraham Robinson's non-standard analysis answers the question of how the completeness theorem can be put to good use in analysis, or that Ax-Kochen theory answers the same question for the theory of ultra products. This book offers two additional examples of such applications of logical metatheorems in specific branches of mathematics.

Part I is devoted to Boolean valued analysis, that is, it is devoted to an application of Scott-Solovay's Boolean valued models of set theory to analysis. Our aim is to establish basic relation between elementary notion in Boolean valued models and analysis.

In Chapter 1, we consider complete Boolean algebras of projections in Hilbert space. Although the algebra of all projections (with their familiar operations) in a Hilbert space does not form a Boolean algebra, many complete Boolean algebras are embedded there.

Let $\mathcal{B}$ be a complete Boolean algebra, let $A$ be a self-adjoint operator with spectral decomposition $A=\int \lambda d E_{\lambda}$. The operator $A$ is said to be in $(\mathcal{B})$ if $E_{\lambda} \in \mathcal{B}$ for every $\lambda$. If $\left\{A_{\alpha}\right\}_{\alpha}$ is a set of pairwise commutable self-adjoint operators, then there exists a complete Boolean algebra $\mathcal{B}$ of projections, such that $A_{\alpha}$ is in $(\mathcal{B})$ for every $\alpha$. The Boolean valued model of such a complete Boolean algebra $\mathcal{B}$ of projections has interesting interpretations in analysis. For example, the real numbers in such a model are exactly the self-adjoint operators in $(\mathcal{B})$ and real number addition, multiplication and order correspond to the addition, multiplication, and order of self-adjoint operators.

The convergence of reals in the model also has a close relation with the convergence of self adjoint operators in the strong topology.

In chapter 2, we consider Boolean valued analysis using a measure algebra which is originally considered by Scott. Reals in this model correspond to measurable functions. We discuss the interpretations of Baire functions, Borel sets, differentiation, integration and the Baire category theorem in the model.

In Part II, we discuss a conservative extension of Peano arithmetic, i.e., an application of Gentzen's cut elimination theorem. Here we take a very weak system of arithmetic with higher type and develop classical analysis in it. The nature of the work is essentially expository. There are many similar works by H. Weyl, G. Kreisel, P. Lorenzen, K. Schütte and many others. We place emphasis on the following points.

1. We take a simple system whose proof-theoretic nature is very clear. The cut elimination theorem implies that the system is a conservative exten- 
sion of Peano arithmetic, i.e., every arithmetical statement proved in the system is a theorem of Peano's arithmetic.

2. We are interested in classical logic and classical analysis. In particular we are interested in classical analytic number theory. For this reason, we devote special attention to the development of the theory of functions of a complex variable.

From this, one can conclude that an arithmetical statement proved by the method of classical analytic number theory is a theorem of Peano's arithmetic.

Since we started this work in the course of correspondence with Kreisel, let us discuss his work on the subject. In his earlier work (part II [3], [4], [5], pp. 168-172, [12], [16]), Kreisel simply transcribed proofs in analysis into the language of arithmetic with free function variables, by approximating the individual functions in analysis, especially analytic number theory, by primitive recursive functions on $\mathbb{Q}$. In the late fifties (part II [9], [10], [11], [13], see also [6] pp. 327-328, 361-362, [7], very readable [8], [15], [17], [18], [19]) he switched to an abstract language, i.e. a language with symbols for higher types but with weak existential axioms. However his main interest was not in conservative extension over Peano's arithmetic, but over the first few levels of the ramified hierarchy. In his lecture (unpublished and unavailable to us), he went back to the old stuff on analytic number theory to show that the latter was conservative over primitive recursive arithmetic. 


\section{Part I}

\section{Boolean Valued Analysis}



In 1963, Paul J. Cohen introduced the notion of forcing and proved many important independency results in set theory. In 1966, D. Scott and R. Solovay reformulated the theory of forcing in terms of Boolean valued models. (A similar idea was developed by Vopenka [12], [13].) We propose to apply the theory of Boolean valued models to analysis. We will introduce the idea of a Boolean valued model but without much explanation. However, the theory is easy to understand if one does several exercises after the basic properties of the model are stated. We will assume the basic properties of self-adjoint operators and their spectral decompositions.

D. Scott not only started Boolean valued models of set theory, with Solovay, but also introduced Boolean valued analysis. His choice of complete Boolean algebras was measure algebras. We will revisit Scott's Boolean valued analysis in Chapter 2.

The idea of Boolean valued models goes back to Church [4] and Rasiowa and Sikorski [8]. However it is fair to say that the success of Scott and Solovay's work has encouraged many similar developments and applications. 



\section{Chapter 1}

\section{Boolean Valued Analysis Using Projection Algebras}

\subsection{Hilbert space}

A bounded operator $P$, of a Hilbert space, is called a projection if $P$ is selfadjoint and $P^{2}=P$. We will use the symbol $I$ to denote the identity operator i.e. $I x=x$ and 0 to denote an operator defined by $0 \cdot x=0$.

A set $\mathcal{B}$ of projections is called a Boolean algebra of projections, if it satisfies the following conditions.

1. Both $I$ and 0 are members of $\mathcal{B}$ and members of $\mathcal{B}$ are pairwise commutable.

2. If $P_{1}$ and $P_{2}$ are members of $\mathcal{B}$, so are $P_{1} \vee P_{2}, P_{1} \wedge P_{2}$, and $\neg P_{1}$, where $P_{1} \vee P_{2}=P_{1}+P_{2}-P_{1} \cdot P_{2}, P_{I} \wedge P_{2}=P_{1} \cdot P_{2}$, and $\neg P_{1}=I-P_{1}$.

A Boolean algebra $\mathcal{B}$ of projections is said to be complete if $\mathcal{B}$ is not only complete as a Boolean algebra but also satisfies the following condition. If $P=\sup P_{\alpha}$, then the range of $P$, denoted by $\mathcal{R}(P)$, is the closure of the linear space spanned by $\bigcup_{\alpha} \mathcal{R}\left(P_{\alpha}\right)$.

From now on, let $\mathcal{B}$ be a complete Boolean algebra of projections. Let $A$ be a self-adjoint operator and let $A=\int \lambda d E_{\lambda}$ be its spectral decomposition. Then $A$ is said to be in $(\mathcal{B})$ if for every real $\lambda, E_{\lambda}$ is a member of $\mathcal{B}$.

Let $A$ and $B$ be self-adjoint and let $A=\int \lambda d E_{\lambda}$ and $B=\int \lambda d E_{\lambda}^{\prime}$ be their spectral decompositions. Then $A$ and $B$ are said to be commutable if for every pair $\lambda, \lambda^{\prime}$ of reals

$$
E_{\lambda} \cdot E_{\lambda^{\prime}}^{\prime}=E_{\lambda^{\prime}}^{\prime} \cdot E_{\lambda}
$$


If $A$ and $B$ are bounded, then the commutativity of $A$ and $B$ is equivalent to $A \cdot B=B \cdot A$.

The following single fact is very useful in our work and will be used without mention.

Lemma 1.1.1. If $\left\{A_{\alpha}\right\}$ be a set of self-adjoint, pairwise commutable operators, then there exists a complete Boolean algebra of projections such that for every $\alpha, A_{\alpha}$ is in $(\mathcal{B})$.

Let $A$ and $B$ be commutable self-adjoint operators. It is usual to define $A+B$ as the operator satisfying the conditions

$$
\begin{aligned}
& \mathcal{D}(A+B)=\mathcal{D}(A) \cap \mathcal{D}(B) \quad \text { and } \\
& \forall x \in \mathcal{D}(A+B) \quad(A+B) x=A x+B x,
\end{aligned}
$$

where $\mathcal{D}(A)$ denotes the domain of $A$. The operator $A+B$, defined in this way, has a unique closed extension. For our purposes we define $A+B$ to be this unique closed extension. The operator $A+B$ is also self-adjoint. In the same way, $A \cdot B$ is defined to be the unique closed extension of the operator which maps $x$, with $x \in \mathcal{D}(B)$ and $B x \in \mathcal{D}(A)$, to $A B x$. The operator $A \cdot B$ is also self-adjoint and $A \cdot B=B \cdot A$. Because of this definition, there is a possibility that $A+B$ and/or $A \cdot B$ is defined on the whole Hilbert space, and therefore bounded, even if $A$ and $B$ are unbounded. In general, if the result of an operator $0(A, B)$ is not closed but has a unique closed extension, we define $0(A, B)$ to be the unique closed extension of the result.

An operator $N$ is said to be normal, if $N=A+i B$ where $A$ and $B$ are self-adjoint and commutable. Also $N^{*}=A-i B$ and $N N^{*}=N^{*} N=A^{2}+B^{2}$. Furthermore, $N$ is said to be in $(\mathcal{B})$, if $A$ and $B$ are in $(\mathcal{B})$. We define $|N|$ to be $\sqrt{A^{2}+B^{2}}$. The operator $|N|$ is self-adjoint.

Let $A$ and $B$ be self-adjoint and commutable. Then $A \leq B$ if and only if for every $x \in \mathcal{D}(A) \cap \mathcal{D}(B),(A x, x) \leq(B x, x)$.

\subsection{The model $V^{(\mathcal{B})}$}

In this section, we summarize the necessary back ground about the Boolean valued model $V^{(\mathcal{B})}$ of set theory. For detail, see $\S 13$. and $\S 16$. in [10], though $+, \cdot,-, \Pi, \Sigma$ are used there as Boolean operations in the place of $\vee, \wedge, \neg$, inf, sup, and $\mathbf{O}$ and $\mathbf{I}$ are used in the place of 0 and $I$.

First we shall give a rough idea of $V^{(\mathcal{B})}$. Let $D$ be a domain. A set of members in $D$ is decided by assigning true or false to $x \in A$ for every member $x$ in $D$. A $\mathcal{B}$-valued set of members in $D$ is decided by assigning any element $P$ in $\mathcal{B}$ to $x \in A$ for every member $x$ in $D$. We write $\llbracket x \in A \rrbracket=P$ if the assigned value of $x \in A$ is $P$. The symbol ' $\llbracket x \in A \rrbracket=P$ ' is read as ' $x \in A$ holds with probability $P$ '. However, ' $\llbracket x \in A \rrbracket=I$ ' is read as ' $x \in A$ holds' and ' $\llbracket x \in A \rrbracket=0$ ' is read as ' $x \in A$ does not hold'.

The universe $V$ of all sets is obtained by starting with the empty set and by creating sets of sets, sets of sets of sets etc. The Boolean valued universe $V^{(\mathcal{B})}$ 
is obtained by starting with the empty set and by creating Boolean valued sets of Boolean valued sets, Boolean valued sets of Boolean valued sets of Boolean valued sets etc. By interpreting true by $I$ and false by 0 , there exists a natural embedding, denoted by $\sqrt{ }$, of $V$ into $V^{(\mathcal{B})}$ i.e.

$$
\sqrt{ }: V \rightarrow V^{(\mathcal{B})}
$$

Let $a \in V$. The corresponding element in $V^{(\mathcal{B})}$ is denoted by $\stackrel{\sqrt{ }}{a}$.

Formally, we shall carry this out as follows. Let $\mathcal{B}$ be a complete Boolean algebra (of projections). For an ordinal $\alpha$, we define $V_{a}^{(\mathcal{B})}$ by transfinite induction as follows:

$$
\begin{aligned}
V_{0}^{(\mathcal{B})} & =\phi \\
V_{\alpha}^{(\mathcal{B})} & =\left\{u \mid u: \mathcal{D}(u) \rightarrow \mathcal{B} \quad \text { and } \mathcal{D}(u) \subseteq \bigcup_{\xi<\alpha} V_{\xi}^{(\mathcal{B})}\right\} \\
\text { and } \quad V^{(\mathcal{B})} & =\bigcup_{\alpha \in \text { On }} V_{\alpha}^{(\mathcal{B})},
\end{aligned}
$$

where On is the class of all ordinal numbers.

It is easy to see that

1) if $\alpha$ is a limit, then $V_{\alpha}^{(\mathcal{B})}=\bigcup_{\xi<\alpha} V_{\xi}^{(\mathcal{B})}$,

2) $V_{\alpha+1}^{(\mathcal{B})}=\left\{u \mid u: \mathcal{D}(u) \rightarrow \mathcal{B}\right.$ and $\left.\mathcal{D}(u) \subseteq V_{\alpha}^{(\mathcal{B})}\right\}$, and

3) if $\alpha \leq \beta$, then $V_{\alpha}^{(\mathcal{B})} \subseteq V_{\beta}^{(\mathcal{B})}$.

For $u, v \in V^{(\mathcal{B})}, \llbracket u \in v \rrbracket$ and $\llbracket u=v \rrbracket$ are defined as functions from $V^{(\mathcal{B})} \times V^{(\mathcal{B})}$ to $\mathcal{B}$ satisfying the following properties.

$$
\begin{aligned}
& \text { 1. } \llbracket u \in v \rrbracket=\sup _{y \in \mathcal{D}(v)}(v(y) \wedge \llbracket u=y \rrbracket) \\
& \text { 2. } \llbracket u=v \rrbracket=\inf _{x \in \mathcal{D}(u)}(u(x) \Rightarrow \llbracket x \in v \rrbracket) \wedge \inf _{y \in \mathcal{D}(v)}(v(y) \Rightarrow \llbracket y \in u \rrbracket)
\end{aligned}
$$

where $P_{1} \Rightarrow P_{2}$ is $\neg P_{1} \vee P_{2}$ i.e.

$$
\left(I-P_{1}\right)+P_{2}-\left(I-P_{1}\right) \cdot P_{2}=I-P_{1}+P_{1} \cdot P_{2} .
$$

In the following, we also use $\wedge, \vee, \neg, \Rightarrow$ as logical connections. Let $\varphi$ be a formula in set theory, that is, let $\varphi$ be obtained by applying logical symbols to atomic formulas of the form $u \in v$ or $u=v$. If $\varphi$ does not contain any free variable and all the constants in $\varphi$ are members in $V^{(\mathcal{B})}$, we define $\llbracket \varphi \rrbracket$ by the following rules.

1. $\llbracket \neg \varphi \rrbracket=\neg \llbracket \varphi \rrbracket=\mathbf{I}-\llbracket \varphi \rrbracket$ 
2. $\llbracket \varphi_{1} \vee \varphi_{2} \rrbracket=\llbracket \varphi_{1} \rrbracket \vee \llbracket \varphi_{2} \rrbracket=\llbracket \varphi_{1} \rrbracket+\llbracket \varphi_{2} \rrbracket-\llbracket \varphi_{1} \rrbracket \cdot \llbracket \varphi_{2} \rrbracket$

3. $\llbracket \varphi_{1} \wedge \varphi_{2} \rrbracket=\llbracket \varphi_{1} \rrbracket \wedge \llbracket \varphi_{2} \rrbracket=\llbracket \varphi_{1} \rrbracket \cdot \llbracket \varphi_{2} \rrbracket$

4. $\llbracket \forall x \varphi(x) \rrbracket=\inf _{u \in V^{(\mathcal{B})}} \llbracket \varphi(u) \rrbracket$

5. $\llbracket \exists x \varphi(x) \rrbracket=\sup _{u \in V^{(\mathcal{B})}} \llbracket \varphi(u) \rrbracket$

From this definition we have the following basic properties.

1. $\llbracket u=u \rrbracket=\mathbf{I}$

2. $\llbracket u=v \rrbracket=\llbracket v=u \rrbracket$

3. $\llbracket u_{1}=u_{2} \rrbracket \cdot \llbracket u_{2}=u_{3} \rrbracket \leq \llbracket u_{1}=u_{3} \rrbracket$

4. $\llbracket u=v \rrbracket \cdot \llbracket \varphi(u) \rrbracket \leq \llbracket \varphi(v) \rrbracket$

5. $\llbracket u=v \rrbracket=\llbracket \forall x(x \in u$ iff $x \in v) \rrbracket$.

Since all the statements in analysis can be expressed in the language of set theory, we can assign a value in $\mathcal{B}$ to any statement in analysis by $\llbracket \rrbracket$.

Let $\mathcal{M}=\mathcal{R}(P)$. Roughly the meaning of $\llbracket \varphi \rrbracket=P$ is that $\varphi$ holds if we restrict our Boolean algebra $\mathcal{B}$ to a subspace $\mathcal{M}$. In another words, the meaning of $\llbracket \varphi \rrbracket=P$ is that $\varphi$ does not hold if we restrict our Boolean algebra $\mathcal{B}$ to a subspace $\mathcal{M}^{\perp}$.

The theorem, which is the base of our work, is the following.

Theorem 1.2.1. The Boolean valued universe $V^{(\mathcal{B})}$ is a model of $Z F$ set theory with the axiom of choice. This set theory we denote by ZFC. In other words, if $\varphi$ is a theorem of $Z F C$, then $\llbracket \varphi \rrbracket=I$ i.e. $\varphi$ holds in $V^{(\mathcal{B})}$.

Since every theorem in contemporary mathematics is a theorem in ZFC, we can express this in the following way. If $\varphi$ is a theorem, then $\varphi$ holds in $V^{(\mathcal{B})}$. Moreover, from the proof of the Theorem 1.2.1, we have the following corollary.

Corollary 1.2.2. If $\varphi$ is a theorem, then ' $\varphi$ holds in $V^{(\mathcal{B})}$ ' is also a theorem.

Since conditional is often used in mathematics, the following equivalence is useful in practice

$$
\begin{aligned}
& \llbracket \varphi_{1} \Rightarrow \varphi_{2} \rrbracket=\left(\llbracket \varphi_{1} \rrbracket \Rightarrow \llbracket \varphi_{2} \rrbracket\right) \quad \text { and } \\
& \left(P_{1} \Rightarrow P_{2}\right)=I \quad \text { iff } \quad P_{1} \leq P_{2} .
\end{aligned}
$$

The following lemma which is Theorem 13.13. in [10] is also useful.

Lemma 1.2.3. For $u \in V^{(\mathcal{B})}$,

$$
\text { 1. } \llbracket \exists x \in u \varphi(x) \rrbracket=\sup _{x \in \mathcal{D}(u)} u(x) \cdot \llbracket \varphi(x) \rrbracket
$$


2. $\llbracket \forall x \in u \varphi(x) \rrbracket=\inf _{x \in \mathcal{D}(u)}(u(x) \Rightarrow \llbracket \varphi(x) \rrbracket)$.

Now we define the embedding $\sqrt{ }: V \rightarrow V^{(\mathcal{B})}$ precisely, by transfinite induction as follows.

For $y \in V$,

$$
\stackrel{\sqrt{ }}{y}=\{\langle\stackrel{\sqrt{x}}{x}, I\rangle \mid x \in y\}
$$

i.e. $\stackrel{v}{y}$ is a constant function such that $\mathcal{D}(\stackrel{\sqrt{y}}{)})=\{\stackrel{v}{x} \mid x \in y\}$ and its value is constantly $I$.

Obviously the following proposition holds.

Proposition 1.2.4 (Theorem 13.17. in [10]). For $x, y \in V$,

$$
\begin{aligned}
& \text { 1. } x \in y \text { iff } \llbracket \stackrel{\sqrt{ }}{x} \in \bar{v} \rrbracket=I \text { and } \\
& x \notin y \text { iff } \llbracket \sqrt{x} \in \stackrel{\sqrt{ }}{ } \rrbracket=0, \\
& \text { 2. } x=y \text { iff } \llbracket \stackrel{\vee}{x}=\stackrel{\vee}{y} \rrbracket=I \text { and }
\end{aligned}
$$

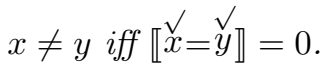

In order to develop analysis in set theory, first the natural numbers are constructed from $\phi$, which is also denoted by 0 , the integers are constructed as pairs of natural numbers, the rational numbers are constructed as pairs of integers, and finally, the real numbers are constructed by Dedekind's cuts of rational numbers. Since $V^{(\mathcal{B})}$ satisfies $\mathrm{ZFC}$, we adopt the same definition of the natural numbers, integers, rational numbers and real numbers so that all the theorems in analysis also hold in $V^{(\mathcal{B})}$. Let us denote the set of all natural numbers by $\omega$, the set of all rational numbers by $\mathbb{Q}$, the set of all real numbers by $\mathbb{R}$ and the set of all complex numbers by $\mathbb{C}$. The first natural question is what are $\omega, \mathbb{Q}, \mathbb{R}$, and $\mathbb{C}$ in $V^{(\mathcal{B})}$. First two are answered as follows. (cf. pp. 129-130 in [10]).

1. Let $n$ be a natural number. Then $n$ in $V^{(\mathcal{B})}$ is $\stackrel{v}{n}$. More precisely there exists a formula $\varphi(x)$ which define $n$ in the sense that $\exists ! x \varphi(x) \wedge \varphi(n)$ is provable. Then $\exists ! x \varphi(x) \wedge \varphi(\sqrt{n})$ holds in $V^{(\mathcal{B})}$. In particular 0 , in $V^{(\mathcal{B})}$, is $\underset{v}{0}$, that is, $\underset{\phi}{ }$ is the empty set in $V^{(\mathcal{B})}$. In $V^{(\mathcal{B})}, \omega$ is $\underset{\omega}{\omega}$.

Moreover $\stackrel{\vee}{n}+\stackrel{\vee}{m}$ and $\stackrel{\vee}{n} \cdot \stackrel{\vee}{m}$, in $V^{(\mathcal{B})}$, are $(n+m) \sqrt{ }$ and $(n \cdot m) \vee$ respectively. Similarly $\stackrel{v}{n} \leq \stackrel{v}{m}$ iff $n \leq m$.

2. Let $r$ be a rational number. Then $r$, in $V^{(\mathcal{B})}$, is $\stackrel{v}{r}$. Moreover $r_{1}+\underset{r_{2}}{\checkmark}=$

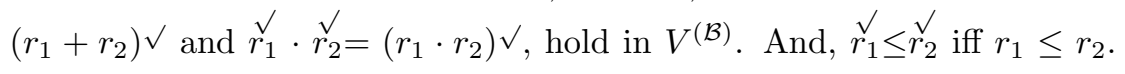


Finally $\mathbb{Q}$, in $V^{(\mathcal{B})}$, is $\stackrel{\vee}{\mathbb{Q}}$. We often write $n, r, \omega$ and $\mathbb{Q}$ in the place of

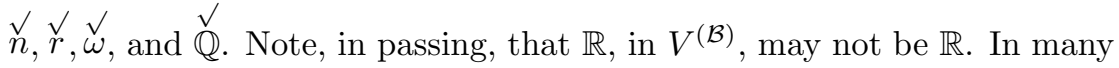
cases, we can prove that $\llbracket \mathbb{R}=\mathbb{R} \rrbracket=0$.

A subset $\left\{P_{\alpha}\right\}$ of $\mathcal{B}$ is called a partition of unity if the following conditions are satisfied.

1. If $\alpha \neq \beta$, then $P_{\alpha} \cdot P_{\beta}=0$.

2. $I=\sum_{\alpha} P_{\alpha}=\sup _{\alpha} P_{\alpha}$,

where $\sum_{\alpha} P_{\alpha}$ is defined in the strong topology. Let $\left\{P_{\alpha}\right\}$ be a partition of unity and let $\left\{u_{\alpha}\right\}$ be a subset of $V^{(\mathcal{B})}$. Then there exists an element $u$ of $V^{(\mathcal{B})}$ such that

$$
\forall \alpha \llbracket u=u_{\alpha} \rrbracket \geq P_{\alpha} .
$$

Moreover if there exists another $u^{\prime}$ such that $\forall \alpha\left(\llbracket u^{\prime}=u_{\alpha} \rrbracket \geq P_{\alpha}\right)$, then $\llbracket u=$ $u^{\prime} \rrbracket=I$, i.e. $u=u^{\prime}$ holds in $V^{(\mathcal{B})}$. We denote this $u$ by $\sum_{\alpha} u_{\alpha} P_{\alpha}$ which is determined uniquely in the sense of equality in $V^{(\mathcal{B})}$. (cf. Theorem 6.9. and Corollary 16.3. in [10]).

Proposition 1.2.5. Let $u=\sum_{\alpha} u_{\alpha} P_{\alpha}$. Then

$$
\llbracket \varphi(u) \rrbracket=\sum_{\alpha} \llbracket \varphi\left(u_{\alpha}\right) \rrbracket \cdot P_{\alpha} .
$$

Proof. Since $u=u_{\alpha} \wedge \varphi\left(u_{\alpha}\right) \Rightarrow \varphi(u)$,

$$
\llbracket u=u_{\alpha} \rrbracket \cdot \llbracket \varphi\left(u_{\alpha}\right) \rrbracket \leq \llbracket \varphi(u) \rrbracket .
$$

So

$$
\llbracket \varphi\left(u_{\alpha}\right) \rrbracket \cdot P_{\alpha}=\llbracket u=u_{\alpha} \rrbracket \cdot \llbracket \varphi\left(u_{\alpha}\right) \rrbracket \cdot P_{\alpha} \leq \llbracket \varphi(u) \rrbracket \cdot P_{\alpha} .
$$

In the same way,

$$
\llbracket \varphi(u) \rrbracket \cdot P_{\alpha}=\llbracket u=u_{\alpha} \rrbracket \cdot \llbracket \varphi(u) \rrbracket \cdot P_{\alpha} \leq \llbracket \varphi\left(u_{\alpha}\right) \rrbracket \cdot P_{\alpha} .
$$

Therefore

$$
\llbracket \varphi(u) \rrbracket=\sum_{\alpha} \llbracket \varphi(u) \rrbracket \cdot P_{\alpha}=\sum_{\alpha} \llbracket \varphi\left(u_{\alpha}\right) \rrbracket \cdot P_{\alpha}
$$

Proposition 1.2.6. Let $u=\sum_{\alpha} u_{\alpha} P_{\alpha}$ and $\forall \alpha, \beta\left(\alpha \neq \beta \Rightarrow \llbracket u_{\alpha}=u_{\beta} \rrbracket=0\right)$. Then

$$
P_{\alpha}=\llbracket u=u_{\alpha} \rrbracket .
$$


Proof.

$$
\llbracket u=u_{\alpha} \rrbracket=\sum_{\beta} \llbracket u_{\beta}=u_{\alpha} \rrbracket \cdot P_{\beta}=P_{\alpha} .
$$

Proposition 1.2.7. Let $u=\sum_{\alpha} u_{\alpha} P_{\alpha}$. Then

$$
\llbracket \varphi(u) \rrbracket=\sum_{\alpha} \llbracket u=u_{\alpha} \rrbracket \cdot \llbracket \varphi\left(u_{\alpha}\right) \rrbracket .
$$

Proof.

$$
\sum_{\alpha} \llbracket u=u_{\alpha} \rrbracket \cdot \llbracket \varphi\left(u_{\alpha}\right) \rrbracket \geq \sum_{\alpha} \llbracket \varphi\left(u_{\alpha}\right) \rrbracket \cdot P_{\alpha}=\llbracket \varphi(u) \rrbracket .
$$

On the other hand

$$
\sum_{\alpha} \llbracket u=u_{\alpha} \rrbracket \cdot \llbracket \varphi\left(u_{\alpha}\right) \rrbracket \leq \llbracket \varphi(u) \rrbracket .
$$

We call $u \in V^{(\mathcal{B})}$ a natural number in $V^{(\mathcal{B})}$, if $\llbracket u \in \omega \rrbracket=I$. Now we would like to see what the natural numbers in $V^{(\mathcal{B})}$ are. Let $\llbracket u \in \omega \rrbracket=\llbracket u \in \mathcal{\omega} \rrbracket=I$. Then

$$
\begin{aligned}
\llbracket u \in \breve{\omega} \rrbracket & =\sup _{x \in \mathcal{D}(\breve{\omega})} \check{\omega}(x) \cdot \llbracket u=x \rrbracket \\
& =\sup _{n \in \omega} \llbracket u=\stackrel{\sqrt{ }}{n} \rrbracket .
\end{aligned}
$$

Let $P_{n}=\llbracket u=\sqrt{ } \rrbracket$. It is easy to see that $\left\{P_{n}\right\}$ is a partition of unity and

$$
u=\sum_{n} \stackrel{\sqrt{n}}{n} P_{n}=\sum_{n} n \cdot P_{n}
$$

There might be many 0's among the $P_{n}$ 's. Usually we take off the 0's and write

$$
u=\sum_{i} n_{i} \cdot P_{i}
$$

where $i$ ranges over a finite or infinite subset of $\omega$.

Let $\left\{P_{i}\right\}$ be a partition of unity, let $\left\{n_{i}\right\}$ be a subset of $\omega$ and let $u=\sum_{i} n_{i} P_{i}$. Then it is easily seen that $\llbracket u \in \omega \rrbracket=I$. Therefore all natural numbers in $V^{(\mathcal{B})}$ are expressed in the form $\sum_{i} n_{i} P_{i}$. This form is very convenient for the following reason. Let $\left\{P_{i}\right\}$ and $\left\{P_{j}^{\prime}\right\}^{i}$ be two partitions of unity. Then $\left\{P_{i} \cdot P_{j}^{\prime}\right\}_{i j}$ is also 
a partition of unity. Let $u=\sum_{i} n_{i} P_{i}$ and $v=\sum_{j} m_{j} P_{j}^{\prime}$. Then the following properties are easily checked.

$$
\begin{aligned}
& u+v=\sum_{i, j}\left(n_{i}+m_{j}\right) P_{i} \cdot P_{j}^{\prime} \\
& u \cdot v=\sum_{i, j}\left(n_{i} \cdot m_{j}\right) P_{i} \cdot P_{j}^{\prime} \\
& \llbracket u<v \rrbracket=\sup \left\{P_{i} \cdot P_{j}^{\prime} \mid n_{i}<m_{j}\right\} .
\end{aligned}
$$

Let $u \in V^{(\mathcal{B})}$. Then $u$ is called a rational number in $V^{(\mathcal{B})}$, if $\llbracket u \in \mathbb{Q} \rrbracket=I$. Since $\mathbb{Q}$ is obtained from $\omega$ by a simple construction, what we proved about $\omega$ is also true for $\mathbb{Q}$, that is, the following properties hold.

1. All rational numbers in $V^{(\mathcal{B})}$ are those of the form $\sum_{i} r_{i} P_{i}$ where $\left\{P_{i}\right\}$ is a partition of unity and $\left\{r_{i}\right\}$ is a subset of $\mathbb{Q}$.

2. Let $u=\sum r_{i} P_{i}$ and $v=\sum_{j} r_{j}^{\prime} P_{j}^{\prime}$ be two rational numbers in $V^{(\mathcal{B})}$. Then

$$
\begin{aligned}
& u+v=\sum_{i, j}\left(r_{i}+r_{j}^{\prime}\right) P_{i} \cdot P_{j}^{\prime} \\
& u \cdot v=\sum_{i, j}\left(r_{i} \cdot r_{j}^{\prime}\right) \cdot P_{i} \cdot P_{j}^{\prime} \\
& \llbracket u \leq v \rrbracket=\sup \left\{P_{i} \cdot P_{j}^{\prime} \mid r_{i} \leq r_{j}^{\prime}\right\} \\
& \llbracket u<v \rrbracket=\sup \left\{P_{i} \cdot P_{j}^{\prime} \mid r_{i}<r_{j}^{\prime}\right\} .
\end{aligned}
$$

Theorem 1.2.8 (The Maximum Principle, Theorem 16.2. in [10]). Let $\varphi$ be a formula. Then there exists a $u \in V^{(\mathcal{B})}$ such that

$$
\llbracket \varphi(u) \rrbracket=\llbracket \exists x \varphi(x) \rrbracket .
$$

Since $\llbracket \exists x \varphi(x) \rrbracket=\sup _{v \in V^{(\mathcal{B})}} \llbracket \varphi(v) \rrbracket$, the meaning of the theorem is that there exists a $u$ which maximizes the value of $\llbracket \varphi(u) \rrbracket$.

Let $\varphi(x)$ be a formula with only $x$ as a free variable. Let $v_{0} \in V^{(\mathcal{B})}$ satisfy $\llbracket \varphi\left(v_{0}\right) \rrbracket=I$. Now consider a statement of the form $\forall x(\varphi(x) \Rightarrow \psi(x))$. Since $\llbracket \forall x(\varphi(x) \Rightarrow \psi(x)) \rrbracket=\inf _{u \in V^{(\mathcal{B})}} \llbracket \varphi(u) \Rightarrow \psi(u) \rrbracket$, we have to calculate $\llbracket \varphi(u) \Rightarrow \psi(u) \rrbracket$ for every $u \in V^{(\mathcal{B})}$ in order to calculate $\llbracket \forall x(\varphi(x) \Rightarrow \psi(x)) \rrbracket$. Let $u \in V^{(\mathcal{B})}$ and $\llbracket \varphi(u) \rrbracket=P$. Define $u^{\prime}=u \cdot P+v_{0} \cdot(I-P)$. It is easily seen that $\llbracket \varphi\left(u^{\prime}\right) \rrbracket=I$ and

$$
\llbracket \psi\left(u^{\prime}\right) \rrbracket \leq(\llbracket \varphi(u) \rrbracket \Rightarrow \llbracket \psi(u) \rrbracket) .
$$

Therefore we have

$$
\llbracket \forall x(\varphi(x) \Rightarrow \psi(x)) \rrbracket=\inf _{\llbracket \varphi(u) \rrbracket=I} \llbracket \psi(u) \rrbracket .
$$


In the same way, we have

$$
\llbracket \exists x(\varphi(x) \wedge \psi(x)) \rrbracket=\sup _{\llbracket \varphi(u) \rrbracket=I} \llbracket \psi(u) \rrbracket .
$$

This justifies the following definition. We define the interpretation of $\{x \mid \varphi(x)\}$ with respect to $V^{(\mathcal{B})}$ to be $\left\{u \in V^{(\mathcal{B})} \mid \llbracket \varphi(u) \rrbracket=I\right\}$, assuming that it is not empty.

The above discussion shows that the interpretation of important notions in analysis is at the center of our work.

Remark. Let $u \in V^{(\mathcal{B})}$. Then the class $\left\{v \in V^{(\mathcal{B})} \mid \llbracket u=v \rrbracket=I\right\}$ is always a proper class. This sometimes causes a technical inconvenience. In the following, we always think that we have picked up a representative from an equivalent class $\left\{v \in V^{(\mathcal{B})} \mid \llbracket u=v \rrbracket=I\right\}$. Technically we use a set $\left\{v \in V^{(\mathcal{B})} \mid v\right.$ is of the lowest rank with $\llbracket u=v \rrbracket=I\}$ to represent a proper class $\left\{v \in V^{(\mathcal{B})} \mid \llbracket u=v \rrbracket=I\right\}$. In this way, we can avoid the use of a uniform choice function.

\subsection{Real numbers in $V^{(\mathcal{B})}$}

We define real numbers by Dedekind cuts. More precisely, by a real number we mean a set of rational numbers which is the upper segment of a Dedekind cut. If $a$ is a real number and $a$ corresponds to a rational number $r$, then we include $r$ in $a$, that is $r$ is the smallest member of $a$. Thus ' $a$ is a real number' is expressed by the following formula. $t \in a))$

$a \subseteq \mathbb{Q} \wedge \exists s \in \mathbb{Q}(s \in a) \wedge \exists s \in \mathbb{Q}(s \notin a) \wedge \forall s \in \mathbb{Q}(s \in a \Leftrightarrow \forall t \in \mathbb{Q}(s<t \Rightarrow$

We define $\mathbb{R}^{(\mathcal{B})}$ to be the interpretation of $\mathbb{R}$ in $V^{(\mathcal{B})}$, i.e.

$$
\mathbb{R}^{(\mathcal{B})}=\left\{u \in V^{(\mathcal{B})} \mid \llbracket u \text { is a real } \rrbracket=I\right\} .
$$

Let $u \in \mathbb{R}^{(\mathcal{B})}$ and $r \in \mathbb{Q}$. We define $P_{r}$ to be $\llbracket r \in u \rrbracket$. Then the following is easily checked.

1. $\inf _{r \in \mathbb{Q}} P_{r}=0$

2. $\sup _{r \in \mathbb{Q}} P_{r}=I$

3. $P_{r}=\inf _{r<s} P_{s}$.

Let $\lambda$ range over real numbers. Let $E_{\lambda}=\inf _{\lambda<r} P_{r}$. Then

1. $\inf _{\lambda} E_{\lambda}=0$

2. $\sup E_{\lambda}=I$

3. $E_{\lambda}=\inf _{\lambda<\mu} E_{\mu}$. 
This implies that $\left\{E_{\lambda}\right\}$ is a resolution of the identity in $(\mathcal{B})$. On the other hand, let $\left\{E_{\lambda}\right\}$ be a resolution of the identity in $(\mathcal{B})$. Let $P_{r}=E_{r}$ for each rational number $r$, and define $u$ to be a function satisfying

$$
\mathcal{D}(u)=\{\check{r} \mid r \in \mathbb{Q}\} \quad \text { and } \quad u(\check{r})=P_{r} .
$$

Then $u \in \mathbb{R}^{(\mathcal{B})}$ is easily checked and the correspondence between $\mathbb{R}^{(\mathcal{B})}$ and the set of all resolutions of the identity in $(\mathcal{B})$ is $1-1$ onto. Since there is a 1-1 onto correspondence between the set of all resolutions of the identity in $(\mathcal{B})$ and the set of all self-adjoint operators in $(\mathcal{B})$, this establishes a 1-1 onto correspondence between the set of all self-adjoint operators in $(\mathcal{B})$ and $\mathbb{R}^{(\mathcal{B})}$. From now on, we express this relation by simply saying that the interpretation of a real number in $V^{(\mathcal{B})}$ is a self-adjoint operator in $(\mathcal{B})$. We are going to investigate the meaning of addition, multiplication and $\leq$ for real numbers in $V^{(\mathcal{B})}$ as operations and a relation for self-adjoint operators in $(\mathcal{B})$.

For $a \in \mathbb{R}, \check{a} \in \mathbb{R}^{(\mathcal{B})}$. Furthermore $\check{a}$ represents a resolution of the identity $\left\{E_{\lambda}\right\}$ such that $E_{\lambda}=0$ if $\lambda<a$ and $E_{\lambda}=I$ if $a \leq \lambda$. Therefore $\check{a}$ represents an operator $a \cdot I$ i.e. $a$ itself.

Though $\omega=\check{\omega}$ and $\mathbb{Q}=\check{\mathbb{Q}}$ hold, $\mathbb{R}=\check{\mathbb{R}}$ does not hold in many Boolean valued universes $V^{(\mathcal{B})}$. For example, take $L^{2}(-\infty, \infty)$, a self-adjoint operator $x$, and any complete Boolean algebra $\mathcal{B}$ such that $x$. is in $(\mathcal{B})$. Let $u \in \mathbb{R}^{(\mathcal{B})}$ correspond to $x$. In order to prove that $\llbracket \mathbb{R}=\check{\mathbb{R}} \rrbracket=0$, it suffices to show that $\llbracket u \in \breve{\mathbb{R}} \rrbracket=0$ since $\llbracket u \in \mathbb{R} \rrbracket=I$. Therefore it suffices to show that $\llbracket \check{a}=u \rrbracket=0$ for every $a \in \mathbb{R}$.

$$
\begin{gathered}
\llbracket \check{a}=u \rrbracket=\inf _{r \in \mathbb{Q}} \llbracket \check{r} \in \check{a} \Leftrightarrow \check{r} \in u \rrbracket \text { and } \\
\llbracket \check{r} \in \check{a} \Leftrightarrow \check{r} \in u \rrbracket= \begin{cases}\llbracket \check{r} \in u \rrbracket & \text { if } a \leq r \\
I-\llbracket \check{r} \in u \rrbracket & \text { if } r<a .\end{cases}
\end{gathered}
$$

Let $x \cdot=\int \lambda d E_{\lambda}$. Then $\llbracket \check{r} \in u \rrbracket=E_{r}$. Therefore $\llbracket \check{a}=u \rrbracket=E_{a}-E_{a-0}=0$. The meaning of this fact is that there are many reals in $V^{(\mathcal{B})}$ that can not be expressed in the form $\sum_{\alpha} a_{\alpha} P_{\alpha}$, where $\left\{P_{\alpha}\right\}$ is a partition of unity and $\left\{a_{\alpha}\right\}$ is a subset of $\mathbb{R}$. It also means that there are lots of self-adjoint operators in $(\mathcal{B})$ which are not of the form $\sum_{\alpha} a_{\alpha} P_{\alpha}$.

Let $u$ and $v$ be in $\mathbb{R}^{(\mathcal{B})}$. Let $A=\int \lambda d E_{\lambda}$ and $B=\int \lambda d E_{\lambda}^{\prime}$ be corresponding self-adjoint operators in $(\mathcal{B})$ respectively. We proceed to see what the basic operations for $u$ and $v$ correspond for $A$ and $B$.

Proposition 1.3.1. The sum $u+v$ corresponds to $A+B$.

Proof. Let $a$ and $b$ be real numbers. The addition for the upper segment of Dedekind cuts is defined in the following way.

$$
r \in(a+b) \text { iff } \forall s \in \mathbb{Q}\left(r<s \Rightarrow \exists t_{1}, t_{2} \in \mathbb{Q}\left(s=t_{1}+t_{2} \wedge t_{1} \in a \wedge t_{2} \in b\right)\right)
$$


Interpreting this in $V^{(\mathcal{B})}, u+v$ corresponds to an operator $\int \lambda d E_{\lambda}^{\prime \prime}$ such that

$$
E_{\lambda}^{\prime \prime}=\inf _{\lambda<\lambda^{\prime}} \sup _{\mu} E_{\mu} \cdot E_{\lambda^{\prime}-\mu}^{\prime}
$$

It is easily checked that $A+B=\int \lambda d E_{\lambda}^{\prime \prime}$.

Proposition 1.3.2. The inequality $u \leq v$ holds in $V^{(\mathcal{B})}$ iff $A \leq B$.

Proof. By definition, $u \leq v$, for two reals defined by Dedekind cuts, if $v \subseteq u$. So

$$
\begin{array}{lll}
\llbracket u \leq v \rrbracket=I \quad \text { iff } & \llbracket r \in \mathbb{Q}(r \in v \Rightarrow r \in u) \rrbracket=I \\
\text { iff } & \forall r \in \mathbb{Q}\left(\left(I-E_{r}^{\prime}\right)+E_{r}-E_{r} \cdot\left(I-E_{r}^{\prime}\right)=I\right) \\
\text { iff } & \forall r \in \mathbb{Q}\left(E_{r}^{\prime}=E_{r} \cdot E_{r}^{\prime}\right) \\
\text { iff } & \forall r \in \mathbb{Q}\left(E_{r}^{\prime} \leq E_{r}\right) \\
\text { iff } & A \leq B .
\end{array}
$$

Proposition 1.3.3. Let $A=\int \lambda d E_{\lambda}$ be in $(\mathcal{B})$. Let $P \in \mathcal{B}$ and $A P=\int \lambda d E_{\lambda}^{\prime}$. Then

$$
E_{\lambda}^{\prime}= \begin{cases}E_{\lambda} \cdot P+(I-P) & \text { if } \lambda \geq 0 \\ E_{\lambda} \cdot P & \text { otherwise }\end{cases}
$$

Proof. Case 1) $P x=x$

$$
\int \lambda d E_{\lambda}^{\prime} x=\int \lambda d E_{\lambda} x=A x
$$

Case 2) $P x=0$

$$
\int \lambda d E_{\lambda}^{\prime} x=\int_{0}^{\infty} \lambda d(I x)=0 .
$$

Proposition 1.3.4. Let $A=\int \lambda d E_{\lambda}$ and $B=\int \lambda d E_{\lambda}^{\prime}$ be in $(\mathcal{B})$ and let $P \in B$. Then

$$
A P \leq B P \quad \text { iff } E_{\lambda}^{\prime} P \leq E_{\lambda} P \text { for every } \lambda .
$$

Proof. $A P \leq B P$ iff $E_{\lambda} \cdot P+(I-P) \geq E_{\lambda}^{\prime} \cdot P+(I-P)$ for each $\lambda \geq 0$ and

$$
E_{\lambda} P \geq E_{\lambda}^{\prime} P \text { iff } E_{\lambda} P \geq E_{\lambda}^{\prime} P \text { for each } \lambda<0
$$


Proposition 1.3.5.

$\llbracket u \leq v \rrbracket \geq P \quad$ iff $\quad A P \leq B P$.

Proof.

$$
\begin{aligned}
& \llbracket u \leq v \rrbracket \geq P \text { iff } \quad \llbracket \forall r \in \mathbb{Q}(r \in v \Rightarrow r \in u) \rrbracket \geq P \\
& \text { iff } \quad \forall r \in \mathbb{Q}\left(\left(I-E_{r}^{\prime}\right)+E_{r}-\left(I-E_{r}^{\prime}\right) \cdot E_{r} \geq P\right) \\
& \text { iff } \quad \forall r \in \mathbb{Q}\left(\left(I-E_{r}\right)\left(I-E_{r}^{\prime}\right)+E_{r} \geq P\right) \\
& \\
&\left(I-E_{r}\right)\left(I-E_{r}^{\prime}\right)+E_{r} \geq P \Rightarrow E_{r} \geq E_{r}^{\prime} P \\
& \Rightarrow E_{r} P \geq E_{r}^{\prime} P .
\end{aligned}
$$

On the other hand

$$
\begin{aligned}
E_{r} P \geq E_{r}^{\prime} P & \Rightarrow\left(I-E_{r}^{\prime}\right) P \geq\left(I-E_{r}\right) P \\
& \Rightarrow\left(I-E_{r}\right)\left(I-E_{r}^{\prime}\right) P \geq\left(I-E_{r}\right) P \\
& \Rightarrow\left(I-E_{r}\right)\left(I-E_{r}^{\prime}\right) P+E_{r} P \geq P
\end{aligned}
$$

Proposition 1.3.6.

$$
\llbracket u=v \rrbracket \geq P \quad \text { iff } \quad A P=B P .
$$

Proof. Immediate from Proposition 1.3.5.

Proposition 1.3.7. Let $\max (u, v)$ correspond to $\int \lambda d E_{\lambda}^{\prime \prime}$. Then $E_{\lambda}^{\prime \prime}=E_{\lambda} \cdot E_{\lambda}^{\prime}$ for every $\lambda$.

Proof. This is obvious from the proof of Proposition 1.3.2.

Definition 1.3.1. We define $\max (A, B)$ to be this $\int \lambda d E_{\lambda}^{\prime \prime}$.

Proposition 1.3.8. The Boolean complement $-u$ corresponds to $-A$.

Proof. Let $C$ correspond to $-u$. Then

$$
\begin{aligned}
\llbracket u+(-u)=0 \rrbracket=I & \Rightarrow A+C=0 \\
& \Rightarrow C=-A .
\end{aligned}
$$

Proposition 1.3.9.

$$
\llbracket|u-v| \leq \check{\varepsilon} \rrbracket \geq P \quad \text { iff } \quad|A-B| \cdot P \leq \varepsilon,
$$

where $\varepsilon$ is a positive real, and

$$
|A-B|=\max (A-B, B-A) .
$$


Proof. $|u-v| \leq \varepsilon$ iff $u \leq v+\varepsilon \wedge v-\varepsilon \leq u$.

Therefore

$$
\begin{array}{cl}
\llbracket|u-v| \leq \check{\varepsilon} \rrbracket \geq P \quad \text { iff } \quad A P \leq B P+\varepsilon P \text { and } B P-\varepsilon P \leq A P \\
\text { iff } \quad|A-B| \cdot P \leq \varepsilon .
\end{array}
$$

Definition 1.3.2. We define $0<A$ by $\forall \lambda \leq 0\left(E_{\lambda}=0\right)$. If $A$ is bounded, then $0<A$ is 'A is positive definite'.

\section{Proposition 1.3.10.}

$$
0<u \text { holds in } V^{(\mathcal{B})} \text { iff } 0<A \text {. }
$$

Proof.

$$
\begin{array}{lll}
\llbracket 0<u \rrbracket=I & \text { iff } \quad \llbracket 0 \leq u \rrbracket=I \wedge \llbracket u<0 \rrbracket=0 \\
& \text { iff } \quad 0 \leq A \wedge \llbracket \exists r \in \mathbb{Q}(r \in \check{0} \wedge r \notin u) \rrbracket=I \\
& \text { iff } \quad 0 \leq A \wedge \sup _{0 \leq r \in \mathbb{Q}}\left(I-E_{r}\right)=I \\
& \text { iff } \quad 0 \leq A \wedge\left(I-E_{0}\right)=I \\
\text { iff } \quad 0 \leq A \wedge E_{0}=0 \\
\text { iff } \quad 0<A .
\end{array}
$$

From the properties of real numbers,

$$
\llbracket u<v \vee u=v \vee v<u \rrbracket=I .
$$

What does this mean for self-adjoint operators ? Let $P_{1}=\llbracket u<v \rrbracket, P_{2}=\llbracket u=$ $v \rrbracket$, and $P_{3}=\llbracket v<u \rrbracket$. Then $\left\{P_{1}, P_{2}, P_{3}\right\}$ is a partition of unity and we know that $A=A P_{1}+A P_{2}+A P_{3}, B=B P_{1}+B P_{2}+B P_{3}$,

$$
\begin{aligned}
& A P_{1}<B P_{1} \\
& A P_{2}=B P_{2}, \quad \text { and } \\
& B P_{3}<A P_{3} .
\end{aligned}
$$

Let $a$ be a non-negative real. Since $|A| \leq a$ is equivalent to ' $A$ is bounded' and $\|A\| \leq a$, it follows that ' $A$ is a bounded operator' is equivalent to

$$
\exists a \in \mathbb{R}(a>0 \wedge \llbracket|u| \leq \check{a} \rrbracket=I)
$$

where $u$ corresponds to $A$.

Proposition 1.3.11. Let $\left\{P_{\alpha}\right\}$ be a partition of unity, let $\left\{u_{\alpha}\right\}$ be a subset of $\mathbb{R}^{(\mathcal{B})}$ and let $u=\sum_{\alpha} u_{\alpha} P_{\alpha}$. If $A_{\alpha}$ corresponds to $u_{\alpha}$, then $A=\sum_{\alpha} A_{\alpha} \cdot P$ corresponds to $u$. 
Proof. Let $v$ correspond to $A$. It suffices to show that $\llbracket u_{\alpha}=v \rrbracket \geq P_{\alpha}$. That is, $A_{\alpha} \cdot P_{\alpha}=A \cdot P_{\alpha}$. But this is obvious.

Proposition 1.3.12. The product $u \cdot v$ corresponds to $A \cdot B$.

Proof. Let $P_{1}=\llbracket 0<u \rrbracket, P_{2}=\llbracket 0=u \rrbracket, P_{3}=\llbracket u<0 \rrbracket, P_{1}^{\prime}=\llbracket 0<v \rrbracket, P_{2}^{\prime}=$ $\llbracket 0=v \rrbracket$, and $P_{3}^{\prime}=\llbracket v<0 \rrbracket$. Then $\left\{P_{i} P_{j}^{\prime}\right\}_{i, j}$ is a partition of the unity. Let $u_{i j}$ correspond to $A \cdot P_{i} P_{j}^{\prime}$ and $v_{i j}$ correspond to $B \cdot P_{i} P_{j}^{\prime}$. Then $u=\sum u_{i j} P_{i} P_{j}^{\prime}, v=$ $\sum v_{i j} P_{i} P_{j}^{\prime}, A=\sum_{i j} A P_{i} P_{j}^{\prime}, B=\sum_{i j} B P_{i} P_{j}^{\prime}, u \cdot v=\sum_{i j}\left(u_{i j} v_{i j}\right) P_{i} P_{j}^{\prime}$, and $A B=$ $\sum_{i j} A B P_{i} P_{j}^{\prime}$. It suffices to show that $u_{i j} v_{i j}$ corresponds to $\left(A P_{i} P_{j}^{\prime}\right) \cdot\left(B P_{i} P_{j}^{\prime}\right)$. Therefore we assume that $(0<A$ or $0=A$ or $A<0)$ and $(0<B$ or $0=B$ or $B<0)$. If $0=A$ or $0=B$, then the theorem is obvious. If $A<0$, then $-A>0$. So we may assume that $A>0$ and $B>0$ i.e. $\llbracket u>0 \rrbracket=I$ and $\llbracket v>0 \rrbracket=I$. Let $\int \lambda d E_{\lambda}^{\prime \prime}$ correspond to $u \cdot v$. If $\lambda \leq 0$, then $E_{\lambda}^{\prime \prime}=0$ and if $\lambda>0$, then

$$
E_{\lambda}^{\prime \prime}=\inf _{\lambda<\mu} \sup _{\nu>0} E_{\nu} \cdot E_{\mu / \nu}^{\prime}
$$

It is easily seen that $\int \lambda d E_{\lambda}^{\prime \prime}$ corresponds to $A \cdot B$.

Let $\left\{P_{i}\right\}$ and $\left\{P_{j}^{\prime}\right\}$ be partitions of unity and let $\left\{n_{i}\right\}$ and $\left\{m_{j}\right\}$ be subsets of $\omega$. Then $\sum_{i} n_{i} P_{i} \leq \sum_{j} m_{j} P_{j}^{\prime}$ iff $n_{i} \leq m_{j}$ for every $i, j$ satisfying $P_{i} P_{j}^{\prime}>0$.

Proposition 1.3.13. Let $u: \omega \rightarrow \mathbb{R}$ hold in $V^{(\mathcal{B})}$, let $u(\check{i})$ correspond to $A_{i}$ for every $i \in \omega$, and let $v \in \mathbb{R}^{(\mathcal{B})}$ correspond to $A$. Then $\llbracket \lim _{i \rightarrow \infty} u(i)=v \rrbracket=I$ iff for every $\varepsilon>0$ there exists a natural number $\sum_{i} n_{i} P_{i}$ in $V^{(\mathcal{B})}$ such that for every natural number $\sum_{j} m_{j} P_{j}^{\prime}$ in $V^{(x)}$ if $\sum_{i} n_{i} P_{i}<\sum_{j} m_{j} P_{j}^{\prime}$ then

$$
\left|A-\sum_{j} A_{m_{j}} P_{j}^{\prime}\right|<\varepsilon
$$

Proof. $\llbracket \lim _{i \rightarrow \infty} u(i)=v \rrbracket=I$

iff $\llbracket \forall \varepsilon \in \mathbb{Q}(\varepsilon>0 \Rightarrow \exists n \in \omega \forall m \in \omega(m \geq n \Rightarrow|u(m)-v|<\varepsilon)) \rrbracket=I$

iff $\forall \varepsilon>0 \exists \sum_{i} n_{i} P_{i} \llbracket \forall m \in \omega\left(m \geq \sum_{i} \check{n_{i}} P_{i} \Rightarrow|u(m)-v|<\check{\varepsilon}\right) \rrbracket=I$

iff $\forall \varepsilon>0 \exists \sum_{i}^{i} \check{n_{i}} P_{i} \forall \sum_{j} \check{m_{j}} P_{j}^{\prime}\left(\sum_{i} \check{n_{i}} P_{i} \leq \sum_{j} \check{m}_{j} P_{j}^{\prime} \Rightarrow \llbracket\left|u\left(\sum_{j} \check{m}_{j} P_{j}^{\prime}\right)-v\right|<\right.$ $\varepsilon \rrbracket=I)$.

Since $u\left(\sum_{i} \check{n_{i}} P_{i}\right)=\sum_{i} u\left(\check{n_{i}}\right) P_{i}$ holds in $V^{(\mathcal{B})}, \llbracket \lim _{i \rightarrow \infty} u(i)=v \rrbracket=I$ 
iff $\forall \varepsilon>0 \exists \sum_{i} \check{n_{i}} P_{i} \forall \sum_{j} \check{m_{j}} P_{j}^{\prime}\left(\sum_{i} \check{n_{i}} P_{i} \leq \sum_{j} \check{m_{j}} P_{j}^{\prime} \Rightarrow\left|A-\sum_{i} A_{n_{i}} P_{i}\right|<\right.$ $\varepsilon)$.

From what we have discussed, it is clear that complex numbers in $V^{(\mathcal{B})}$ are normal operators in $(\mathcal{B})$. If $u$ corresponds to $A+i B$ and $A$ and $B$ are self-adjoint operators, then $\bar{u}$ (the conjugate of $u$ ) corresponds to $A-i B$ and $|u|$ corresponds to $\sqrt{A^{2}+B^{2}}$. The condition that $u$ corresponds to a bounded normal operator is that there exists a real number a such that $|u|<\check{a}$ holds in $V^{(\mathcal{B})}$. The condition that $u$ corresponds to a unitary operator in $(\mathcal{B})$ is that $u$ belongs to the unit circle in $V^{(\mathcal{B})}$. We have a straightforward generalization here for every property we have discussed for real numbers in $V^{(\mathcal{B})}$.

Remark. $\mathbb{R}$ in $V^{(\mathcal{B})}$ can be represented by $\mathbb{R}^{(\mathcal{B})} \times\{I\}$ i.e. a function whose domain is $\mathbb{R}^{(\mathcal{B})}$ and whose value is constantly $I$.

\subsection{The interpretation of elementary theorems}

By Corollary 1.2.2, " $\varphi$ holds in $V^{(\mathcal{B}) " ~ i s ~ a l s o ~ a ~ t h e o r e m ~ i f ~} \varphi$ is a theorem. This is a machinery to produce another theorem from a theorem. As elementary exercises, we are going to see what kind of theorems we get by this method in very simple cases. Namely we are going to produce theorems by interpreting the first four most elementary theorems in classical analysis i.e. the Bolzano-Weierstrass Theorem, the Intermediate Value Theorem, the Maximum Principle, and Rolle's Theorem in $V^{(\mathcal{B})}$.

First we shall state two general theorems. Let $D \subseteq V^{(\mathcal{B})}$. A function $g: D \rightarrow V^{(\mathcal{B})}$ is called extensional if

$$
\forall d, d^{\prime} \in D \llbracket d=d^{\prime} \rrbracket \leq \llbracket g(d)=g\left(d^{\prime}\right) \rrbracket .
$$

Let $u \in V^{(\mathcal{B})}$. Then $u$ is said to be definite, if

$$
\forall d \in \mathcal{D}(u) \quad u(d)=I .
$$

Proposition 1.4.1 (Theorem 16.8. in [10]). Let $u, v \in V^{(\mathcal{B})}$ be definite and let $\varphi: \mathcal{D}(u) \rightarrow \mathcal{D}(v)$ be an extensional function. Then there exists an $f \in V^{(\mathcal{B})}$ such that

$$
\llbracket f: u \rightarrow v \rrbracket=I \quad \text { and } \forall d \in \mathcal{D}(u) \llbracket f(d)=\varphi(d) \rrbracket=I .
$$

Let $\hat{v}=\{u \mid \llbracket u \in v \rrbracket=I\}$ and $v^{\prime}: \hat{v} \rightarrow\{I\}$, that is, $v^{\prime}$ is a function whose value is constantly $I$. Then

$$
\llbracket v=v^{\prime} \rrbracket=I .
$$

Proposition 1.4.2. Let $u$ and $v$ be definite and $D=\mathcal{D}(u)$. There is a 1-1 correspondence between f's satisfying

$$
\llbracket f: u \rightarrow v \rrbracket=I
$$


and extensional maps $\varphi: D \rightarrow \hat{v}$. The correspondence is given by

$$
\forall d \in D \llbracket f(d)=\varphi(d) \rrbracket=I .
$$

Proof. Proposition 1.4.1 shows how to find $f$ from $\varphi$. In order to find $\varphi$ from $f$, take an arbitrary $d$ from $D$. Then

$$
\llbracket f(d) \in v \rrbracket=I .
$$

There exists an element $c \in \hat{v}$ such that $\llbracket f(d)=c \rrbracket=I$. If $\varphi(d)=c$, then obviously $\varphi$ is extensional and $\llbracket f(d)=\varphi(d) \rrbracket=I$.

Now we consider an interpretation of the Bolzano-Weierstrass Theorem.

Let $A_{0}, A_{1}, \ldots$ and $B$ be pairwise commutable self-adjoint operators with $\left|A_{i}\right| \leq B$ for every $i$. Let $u_{0}, u_{1}, \ldots, v$ correspond to $A_{0}, A_{1}, \ldots, B$ respectively. Let $\mathcal{B}$ be a complete Boolean algebra such that $A_{0}, A_{1}, \ldots, B$ are in $(\mathcal{B})$. Define $\varphi$ by $\varphi:\{\check{n} \mid n \in \omega\} \rightarrow \mathbb{R}^{(\mathcal{B})}$ and $\varphi(\check{n})=u_{n}$ for each $n$. Then $\varphi$ is extensional. Therefore there exists a $u$ such that $\llbracket u: \omega \rightarrow \mathbb{R} \rrbracket=I$ and

$$
\llbracket u(\check{n})=u_{n} \rrbracket=I \quad \text { for each } n .
$$

It is easily seen that $\llbracket \forall n \in \omega|u(n)| \leq v \rrbracket=I$. Therefore there exists a cluster point $v_{0} \in \mathbb{R}^{(\mathcal{B})}$ of $u(n)$, that is,

$$
\llbracket\left|v_{0}\right| \leq v \wedge \forall \varepsilon \in \mathbb{Q}\left(\varepsilon>0 \Rightarrow \forall n \in \omega \exists m \in \omega n \leq m \wedge\left|v_{0}-u(m)\right|<\varepsilon\right) \rrbracket=I .
$$

By interpreting this, we have the following theorem.

Theorem 1.4.3. Let $A_{0}, A_{1}, \ldots$, and $B$ be pairwise commutable self-adjoint operators with $\left|A_{i}\right| \leq B$ for every $i$. Then there exists a self-adjoint operator $A$ such that

1. A commutes with each $A_{i}$ and $B$, and $|A| \leq B$,

2. for every $\varepsilon>0$ and every $n$, there exist $\left\{m_{j}\right\}$ and $\left\{P_{j}\right\}$ such that

2.1. $\left\{P_{j}\right\}$ is a partition of unity and each member of $\left\{P_{j}\right\}$ commutes with each $A_{i}, A$ and $B$,

2.2. $n \leq m_{j}$ for each $j$ and

$$
\left|A-\sum_{j} A_{m_{j}} P_{j}\right|<\varepsilon .
$$

Let $f(x)$ be a continuous function from $\mathbb{R}$ to $\mathbb{R}$ and let $A$ be a self-adjoint operator in $(\mathcal{B})$. Obviously $f(A)$ is also in $(\mathcal{B})$. For every $u \in \mathbb{R}^{(\mathcal{B})}$, define $\varphi(u) \in \mathbb{R}^{(\mathcal{B})}$ to correspond to $f(A)$, where $A$ correspond to $u$. We claim that $\varphi$ is extensional, that is,

$$
\llbracket u=v \rrbracket \leq \llbracket \varphi(u)=\varphi(v) \rrbracket \text { for } u, v \in \mathbb{R}^{(\mathcal{B})} .
$$


Let $A$ and $B$ correspond to $u$ and $v$ respectively. Then it suffices to show that

$$
A P=B P \Rightarrow f(A) P=f(B) P .
$$

Let $A=\int \lambda d E_{\lambda}$ and $B=\int \lambda d E_{\lambda}^{\prime}$. Since $A P=B P$, we have $E_{\lambda} P=E_{\lambda}^{\prime} P$ for every $\lambda$. Suppose $x \in \mathcal{D}(f(A)) \cap \mathcal{D}(f(B))$ and $x=P x$. Then $E_{\lambda} x=E_{\lambda}^{\prime} x$ for every $\lambda$.

$$
f(A) \cdot x=\int f(\lambda) d E_{\lambda} x=\int f(\lambda) d E_{\lambda}^{\prime} x=f(B) \cdot x .
$$

This proves our claim. Therefore $\varphi$ determines a function in $V^{(\mathcal{B})}$. Let us denote this function by $f$ again i.e.

$$
\begin{gathered}
\llbracket f: \mathbb{R} \rightarrow \mathbb{R} \rrbracket=I \text { and } \\
\llbracket f(u)=\varphi(u) \rrbracket=I \text { for every } u \in \mathbb{R}^{(\mathcal{B})} .
\end{gathered}
$$

Now we are going to show that

$$
\begin{array}{r}
\llbracket f \text { is continuous } \rrbracket=I \text { i.e. } \\
\llbracket \forall x \in \mathbb{R} \forall \varepsilon \in \mathbb{Q}(\varepsilon>0 \Rightarrow \exists \delta \in \mathbb{Q}(\delta>0 \wedge \\
\forall y \in \mathbb{R}(|x-y|<\delta \supset|f(x)-f(y)|<\varepsilon))) \rrbracket=I .
\end{array}
$$

Therefore it suffices to show that for every self-adjoint operator $A$ and for every $\varepsilon>0$

$$
\llbracket \exists \delta \in \mathbb{Q}(\delta>0 \wedge \forall y \in \mathbb{R}(|u-y|<\delta \supset|f(u)-f(y)|<\varepsilon)) \rrbracket=I,
$$

where $u$ corresponds to $A$. There exists a partition $\left\{P_{i}\right\}$ of unity and $\left\{r_{i}\right\}$, $\left\{s_{i}\right\} \subseteq \mathbb{Q}$ such that

$$
\sum_{i} r_{i} P_{i}<A<\sum_{i} s_{i} P_{i}
$$

Since $A=\sum_{i} A P_{i}$ and $r_{i} P_{i}<A P_{i}<s_{i} P_{i}$, we may assume that $r<A<s$.

Take $\delta>0$ such that

$$
\forall x \in[r, s] \quad \forall y(|x-y| \leq \delta \Rightarrow|f(x)-f(y)| \leq \varepsilon) .
$$

Let

$$
A=\int \lambda d E_{\lambda} \text { and } B=\int \lambda d E_{\lambda}^{\prime} \text { with }|A-B| \leq \delta .
$$

Then

$$
A-\delta \leq B \leq A+\delta .
$$

Since the spectral decomposition of $A-\delta$ is $\int \lambda d E_{\lambda+\delta}$,

$$
E_{\lambda+\delta} \geq E_{\lambda}^{\prime} \geq E_{\lambda-\delta}
$$


Therefore

$$
\int f(\lambda) d E_{\lambda-\delta} \leq \int f(\lambda) d E_{\lambda}^{\prime} \leq \int f(\lambda) d E_{\lambda+\delta}
$$

Now

$$
\begin{aligned}
f(A)-\varepsilon & =\int f(\lambda-\delta) d E_{\lambda-\delta}-\varepsilon \\
& =\int(f(\lambda-\delta)-\varepsilon) d E_{\lambda-\delta} \\
& \leq \int f(\lambda) d E_{\lambda-\delta}
\end{aligned}
$$

and in the same way,

$$
\int f(\lambda) d E_{\lambda+\delta} \leq f(A)+\varepsilon
$$

So we have

$$
f(A)-\varepsilon \leq f(B) \leq f(A)+\varepsilon .
$$

Now we can interpret $f(A)$ as follows.

The function $f$ itself immediately induce a function

$$
\check{f}: \check{\mathbb{R}} \rightarrow \check{\mathbb{R}} \text {. }
$$

Since $\check{\mathbb{R}}$ is dense in $\mathbb{R}$ in $V^{(\mathcal{B})}$ and $\check{f}$ is uniformly continuous in any closed interval $\left[\sum r_{i} P_{i}, \sum s_{i} P_{i}\right], f$ can be uniquely extended to the whole of $\mathbb{R}$ in $V^{(\mathcal{B})}$. The value of this extended function at $A$ is $f(A)$. Now we have an interpretation of the intermediate value theorem.

Theorem 1.4.4. Let $f(x)$ be continuous and $A$ and $B$ be mutually commutable self-adjoint operators with $A \leq B$. Let $Y$ be a self-adjoint operator which commutes with $A$ and $B$ and let $f(A) \leq Y \leq f(B)$. Then there exists a self-adjoint operator $X$ such that $X$ commutes with $A, B$ and $Y, A \leq X \leq B$, and $Y=f(X)$.

Proof. Let $\mathcal{B}$ be a complete Boolean algebra such that $A, B$, and $Y$ are in $(\mathcal{B})$.

Let $u, v, t \in \mathbb{R}^{(\mathcal{B})}$ correspond to $A, B$, and $Y$ respectively. In $V^{(\mathcal{B})} f$ is continuous on $[u, v]$ and $f(u) \leq t \leq f(v)$. Therefore there exists an $s \in[u, v]$ such that $t=f(s)$. By interpreting this, we get the $X$ in the theorem.

For the interpretation of the maximum principle, we use the following lemma.

Lemma 1.4.5 (Shoenfield's Absoluteness Lemma). Let $M_{0}$ and $M_{1}$ be two transitive models of $Z F C$ with $M_{0} \subseteq M_{1}$ and $u_{1}, \ldots, u_{n}$ be reals in $M_{0}$. If $M_{0}$ has all countable ordinals and $\phi$ is a $\Sigma_{2}^{1}$-formula, then $M_{0} \models \phi\left(u_{1}, \ldots, u_{n}\right)$ iff $M_{1} \models \phi\left(u_{1}, \ldots, u_{n}\right)$.

By a $\Sigma_{2}^{1}$-formula, we mean a formula of the form 
$\exists x \in \mathbb{R} \forall y \in \mathbb{R} \psi\left(x, y, a_{1}, \ldots, a_{n}\right)$ where $\psi$ is arithmetical. A formula of the form $\forall x \in \mathbb{R} \exists y \in \mathbb{R} \psi\left(x, y, a_{1}, \ldots, a_{n}\right)$ with an arithmetical $\psi$ is called a $\Pi_{2}^{1}$-formula. The above lemma also holds if $\phi$ is a $\Pi_{2}^{1}$-formula.

Shoenfield's lemma is translated into the following form for Boolean valued models. (Cf. Theorem 9. 37. in [10]).

Lemma 1.4.6 (Absoluteness Lemma for Boolean valued models). Let $\mathcal{B}_{0} \subseteq \mathcal{B}_{I}$ and $u_{1}, \ldots, u_{n} \in \mathbb{R}^{\left(\mathcal{B}_{0}\right)}$. If $\phi$ is $\Sigma_{2}^{1}$ or $\Pi_{2}^{1}$, then

$$
\llbracket \phi\left(u_{1}, \ldots, u_{n}\right) \rrbracket_{\mathcal{B}_{0}}=\llbracket \phi\left(u_{1}, \ldots, u_{n}\right) \rrbracket_{\mathcal{B}_{1}} .
$$

Here $\llbracket \rrbracket_{\mathcal{B}_{0}}$ is the value calculated with respect to $V^{\left(\mathcal{B}_{0}\right)}$ and $\llbracket \rrbracket_{\mathcal{B}_{1}}$ is the value calculated with respect to $V^{\left(\mathcal{B}_{1}\right)}$.

Now, the next theorem is an interpretation of the maximum principle.

Theorem 1.4.7. ${ }^{*}$ Let $A$ and $B$ be pairwise commutable self-adjoint operators and $f(X)$ be continuous and $A \leq B$. Let $\mathcal{B}$ be the smallest complete Boolean algebra such that $A$ and $B$ are in $(\mathcal{B})$. Then there exists a self-adjoint operator $X$ in $(\mathcal{B})$ such that $A \leq X \leq B$ and $f(Y) \leq f(X)$ for every self-adjoint operator $Y$ which commutes $A$ and $B$ and satisfies $A \leq Y \leq B$.

Proof. Let $u$ and $v \in \mathbb{R}^{(\mathcal{B})}$ correspond to $A$ and $B$ respectively. Then $f$ attains the maximum in $[u, v]$ in $V^{(\mathcal{B})}$. Let $u_{0}$ be a point in $[u, v]$ where $f$ attains the maximum and $X \in \mathbb{R}^{(\mathcal{B})}$ correspond to $u_{0}$.

Now let $Y$ commute with $A$ and $B$ and satisfy $A \leq Y \leq B$. Let $\mathcal{B}_{1}$ be the smallest complete Boolean algebra such that $A, B$, and $Y$ are in $\left(\mathcal{B}_{1}\right)$. Obviously $\mathcal{B} \subseteq \mathcal{B}_{1}$. Since a continuous function can be represented by a single number, $f$ can be considered as a real number. Let $\phi\left(u_{0}, u, v, f\right)$ be the statement " $f$ attains the maximum at $u_{0}$ in $[u, v]$ ". Then by the Absoluteness Lemma, we have

$$
\llbracket \phi\left(u_{0}, u, v, f\right) \rrbracket_{\mathcal{B}_{1}}=\llbracket \phi\left(u_{0}, u, v, f\right) \rrbracket_{\mathcal{B}}=I .
$$

Let $u_{1}$ correspond to $Y$. Then from $\llbracket \phi\left(u_{0}, u, v, f\right) \rrbracket_{\mathcal{B}_{1}}=I$ follows

$$
\llbracket f\left(u_{1}\right) \leq f\left(u_{0}\right) \rrbracket_{\mathcal{B}_{1}}=I .
$$

Theorem 1.4.8. Let $\left\{X_{\alpha}\right\}, A$, and $B$ be pairwise commutable self-adjoint operators and $f(X)$ be continuous and $A \leq X_{\alpha} \leq B$. for every $\alpha$. Let $\mathcal{B}$ be the smallest complete Boolean algebra such that all $X_{\alpha}, A$, and $B$ are in $(\mathcal{B})$. Then there exists a self-adjoint operator $X$ in $(\mathcal{B})$ such that $A \leq X \leq B, \forall \alpha\left(f\left(X_{\alpha}\right) \leq f(X)\right)$ and for every $\mathcal{B}_{1}$ with $\mathcal{B} \subseteq \mathcal{B}_{1}$ and for every $Y$ in $\left(\mathcal{B}_{1}\right)$ with $A \leq Y \leq B$ and $\forall \alpha\left(f\left(X_{\alpha}\right) \leq f(Y)\right)$,

$$
f(X) \leq f(Y) .
$$

${ }^{*}$ Theorems 1.4.7 and 1.4.8 have been pointed out by E. Nelson. The main tool of his proof is the spectral theorem. 
Proof. Let $u_{0}, v_{0}$, and $w_{\alpha}$ correspond to $A, B$, and $X_{\alpha}$ respectively. Define $\tilde{w}$ by the following equations.

$$
\mathcal{D}(\tilde{w})=\text { the set of all } w_{\alpha},
$$

and

$$
\tilde{w}\left(w_{\alpha}\right)=I \text { for every } w_{\alpha} \text { in } \mathcal{D}(\tilde{w}) .
$$

Then we have $\llbracket \tilde{w} \subseteq\left[u_{0}, v_{0}\right] \rrbracket_{\mathcal{B}}=I$ and $\llbracket \exists x \in\left[u_{0}, v_{0}\right](\forall y \in \tilde{w}(f(y) \leq f(x)) \wedge \forall z \in$ $\left[u_{0}, v_{0}\right](\forall y \in \tilde{w}(f(y) \leq f(z)) \Rightarrow f(x) \leq f(z)) \rrbracket_{\mathcal{B}}=I$. Let $u \in \mathbb{R}^{(\mathcal{B})}$ satisfy $\llbracket u \in\left[u_{0}, v_{0}\right] \rrbracket_{\mathcal{B}}=I$ and $\llbracket \forall y \in \tilde{w}(f(y) \leq f(u)) \wedge \forall z \in\left[u_{0}, v_{0}\right](\forall y \in \tilde{w}(f(y) \leq$ $f(z)) \Rightarrow f(u) \leq f(z)) \rrbracket_{\mathcal{B}}=I$, and $X$ correspond to $u$.

Define

$$
P_{1}=\llbracket \exists x \in \tilde{w} \forall y \in \tilde{w}(f(y) \leq f(x)) \rrbracket_{\mathcal{B}}
$$

and

$$
P_{2}=\llbracket \forall x \in \tilde{w} \exists y \in \tilde{w}(f(x)<f(y)) \rrbracket_{\mathcal{B}} .
$$

Then $\left\{P_{1}, P_{2}\right\}$ is a partition of the unity. Therefore we may assume without loss of the generality that either $P_{1}=I$ or $P_{2}=I$ holds.

Case 1) $\llbracket \exists x \in \tilde{w} \forall y \in \tilde{w}(f(y) \leq f(x)) \rrbracket_{\mathcal{B}}=I$. There exists $u \in \mathbb{R}^{(\mathcal{B})}$ such that $\llbracket u \in \tilde{w} \rrbracket_{\mathcal{B}}=I$ and $\llbracket \forall y \in \tilde{w}(f(y) \leq f(u)) \rrbracket_{\mathcal{B}}=I$. Therefore $u$ must be of the form $\sum_{\alpha} w_{\alpha} \cdot P_{\alpha}$ where $\left\{P_{\alpha}\right\}$ is a partition of the unity in $\mathcal{B}$. Let $X$ be $\sum_{\alpha} X_{\alpha} \cdot P_{\alpha}$. For every $Y$ in $\left(\mathcal{B}_{1}\right)$ with $A \leq Y \leq B$ and $\forall \alpha\left(f\left(X_{\alpha}\right) \leq f(Y)\right)$, $f(X) \cdot P_{\alpha} \leq f\left(X_{\alpha}\right) \cdot P_{\alpha} \leq f(Y) \cdot P_{\alpha}$. Therefore we have $f(X) \leq f(Y)$.

Case 2) $\llbracket \forall x \in \tilde{w} \exists y \in \tilde{w}(f(x)<f(y)) \rrbracket_{\mathcal{B}}=I$. Let $a$ be $\{\gamma \in \mathbb{Q} \mid \exists y \in \tilde{w}(\gamma \leq$ $f(y))\}$ in $V^{(\mathcal{B})}$. Obviously $a$ is a subset of $\mathbb{Q}$ in $V^{(\mathcal{B})}$ and can be represented by a real in $V^{(\mathcal{B})}$. Then there exists a $u \in \mathbb{R}^{(\mathcal{B})}$ such that

$$
\llbracket f(u)=\sup a \rrbracket_{\mathcal{B}}=I \text { and } \llbracket u \in\left[u_{0}, v_{0}\right] \rrbracket_{\mathcal{B}}=I .
$$

Now let $\mathcal{B} \subseteq \mathcal{B}_{1}, Y$ be in $\left(\mathcal{B}_{1}\right), A \leq Y \leq B$, and $\forall \alpha\left(f\left(X_{\alpha}\right) \leq f(Y)\right)$.

Since $\llbracket f(u)=\sup a \rrbracket_{\mathcal{B}}=I$, we have by absoluteness Lemma

$$
\llbracket f(u)=\sup a \rrbracket_{\mathcal{B}_{1}}=I .
$$

Let $\llbracket \gamma \in a \rrbracket_{\mathcal{B}_{1}}=I$ and $Z$ correspond to $\gamma$. Then there exists a partition of unity $\left\{P_{\alpha}^{\prime}\right\}$ in $\mathcal{B}_{1}$ such that $Z \leq \sum_{\alpha} f\left(X_{\alpha}\right) \cdot P_{\alpha}^{\prime}$.

Therefore $Z \leq f(Y)$ i.e.

$$
\llbracket \sup a \leq f(y) \rrbracket_{\mathcal{B}_{1}}=I
$$

where $y$ correspond to $Y$. Let $X$ correspond to $u$. Then we have

$$
\llbracket f(u) \leq f(y) \rrbracket_{\mathcal{B}_{1}}=I \text { and } f(X) \leq f(Y) .
$$


Now let $f(x)$ be differentiable and $f^{\prime}(x)$ be continuous. Let $A$ be self-adjoint and let $u$ and $v$ correspond to $A$ and $f^{\prime}(A)$ respectively. Then we are going to show that

$$
\llbracket f^{\prime}(u)=v \rrbracket=I .
$$

Without loss of generality, we may assume that there exist rational numbers $r$ and $s$ such that

$$
r<A<s .
$$

By a routine interpretation, it suffices to show that for every $\varepsilon>0$ there exists a $\delta>0$ such that if $0<|B-A|<\delta$, then

$$
\left|\frac{f(B)-f(A)}{B-A}-f^{\prime}(A)\right| \leq \varepsilon
$$

where $A$ and $B$ are in $(\mathcal{B})$. Take $\delta>0$ such that for every $x \in[r, s]$ and for every $y$, if $0<|y-x|<\delta$, then

$$
\left|\frac{f(y)-f(x)}{y-x}-f^{\prime}(x)\right| \leq \varepsilon .
$$

There exists a self-adjoint operator $C=\int \lambda d E_{\lambda}$ and functions $g(x)$ and $h(x)$ such that $g$ and $h$ are measurable with respect to $\left\{E_{\lambda}\right\}, A=\int g(\lambda) d E_{\lambda}, B=$ $\int_{\text {Now }} h(\lambda) d E_{\lambda}$ and $0<|g(x)-h(x)|<\delta$ almost everywhere with respect to $\left\{E_{\lambda}\right\}$.

$$
\begin{aligned}
\left|\frac{f(B)-f(A)}{B-A}-f^{\prime}(A)\right| & =\left|\int\left(\frac{f(h(\lambda))-f(g(\lambda))}{h(\lambda)-g(\lambda)}-f^{\prime}(g(\lambda)) d E_{\lambda}\right)\right| \\
& \leq \int \varepsilon d E_{\lambda}=\varepsilon .
\end{aligned}
$$

Remark. We can express this fact as follows. The derivative of $f(X)$ at $A$ is $f^{\prime}(A)$. Without assuming the continuity of $f^{\prime}(x)$, one can show the differentiability of $f(X)$ at $A$. However it is an open problem without the continuity of $f^{\prime}(x)$ that the derivative of $f(X)$ at $A$ is $f^{\prime}(A)$.

In the same way as before, we have the following interpretation of Rolle's Theorem.

Theorem 1.4.9. Let $f(x)$ be differentiable and $f^{\prime}(x)$ be continuous and let $A$ and $B$ be mutually commutable self-adjoint operator with $A \leq B$. Then there exists a self-adjoint operator $X$ such that $X$ commutes with $A$ and $B$, $A \leq X \leq B$ and

$$
f(B)-f(A)=(B-A) f^{\prime}(X) .
$$




\subsection{Miscellaneous interpretations}

Let $A$ be a normal operator in $(\mathcal{B})$ and let $\lambda_{0}$ be a complex number. Let

$$
\mathbb{C}^{(\mathcal{B})}=\left\{u \in V^{(\mathcal{B})} \mid \llbracket u \text { is a complex number } \rrbracket=I\right\}
$$

and let $u \in \mathbb{C}^{(\mathcal{B})}$ correspond to $A$. Suppose $\llbracket \check{\lambda_{0}}-u=0 \rrbracket=0$. Then

$$
\llbracket \frac{1}{\check{\lambda_{0}-u}} \text { exists } \rrbracket=I \text {. }
$$

Let $B \in \mathbb{C}^{(\mathcal{B})}$ correspond to $\frac{1}{\check{\lambda_{0}-u}}$. Then $\left(\lambda_{0} I-A\right) \cdot B=I$. Now suppose that $\frac{1}{\lambda_{0} I-A}$ does not exist with positive probability i.e. $\llbracket \check{\lambda_{0}}-u=0 \rrbracket=P>0$. This means that $\lambda_{0} P=A P$. Let $A=A_{1}+i A_{2}=\int \lambda d E_{\lambda}+i \int \lambda d E_{\lambda}^{\prime}$ and $\lambda_{0}=a+i b$, where $a$ and $b \in \mathbb{R}$. Then $\lambda_{0} P=A P$ implies that

$$
E_{\lambda} \cdot P=0 \text { if } \lambda<a
$$

and

$$
E_{\lambda} \cdot P=P \text { if } a \leq \lambda
$$

that is,

$$
E_{a}-E_{a-0} \geq P
$$

In the same way, we have $E_{b}^{\prime}-E_{b-0}^{\prime} \geq P$. This means that $\lambda_{0}$ is a point spectrum of $A$.

Next we consider the case that $\frac{1}{\lambda_{0} I-A}$ exists but is not bounded. This means that for every $\varepsilon>0$

$$
\llbracket \frac{1}{\left|\check{\lambda_{0}}-u\right|}>\frac{1}{\varepsilon} \rrbracket>0 \text { i.e. } \llbracket\left|\check{\lambda_{0}}-u\right|<\check{\varepsilon} \rrbracket>0 .
$$

Since $\varepsilon$ is arbitrarily small, this is equivalent to for every $\varepsilon>0$,

$$
\llbracket\left|\check{a}-u_{1}\right| \leq \check{\varepsilon} \rrbracket \cdot \llbracket\left|\check{b}-u_{2}\right| \leq \check{\varepsilon} \rrbracket>0,
$$

where $u_{1}, u_{2} \in \mathbb{R}^{(\mathcal{B})}$ and $\llbracket u=u_{1}+i u_{2} \rrbracket=I$. Suppose

$$
\llbracket\left|\check{a}-u_{1}\right| \leq \check{\varepsilon} \rrbracket \cdot \llbracket\left|\check{b}-u_{2}\right| \leq \check{\varepsilon} \rrbracket=P>0 .
$$

Then

$$
\begin{gathered}
\llbracket \check{a}-\check{\varepsilon} \leq u_{1} \leq \check{a}+\check{\varepsilon} \rrbracket \geq P \text { and } \\
\llbracket \check{b}-\check{\varepsilon} \leq u_{2} \leq \check{b}+\check{\varepsilon} \rrbracket \geq P .
\end{gathered}
$$


So

$$
E_{\lambda} \cdot P=0 \text { if } \lambda<a-\varepsilon
$$

and

$$
E_{\lambda} \cdot P=P \text { if } \lambda \geq a+\varepsilon
$$

that is,

$$
E_{a+\varepsilon}-E_{a-\varepsilon-0} \geq P .
$$

In the same way $E_{b+\varepsilon}^{\prime}-E_{b-\varepsilon-0} \geq P$. This means that $a+b i$ is a continuous spectrum of $A$.

Let $A=\int \lambda d E_{\lambda}$ be in $(\mathcal{B})$, let $u \in \mathbb{R}^{(\mathcal{B})}$ correspond to $A$ and let $a, \varepsilon \in \mathbb{R}$ with $\varepsilon>0$. If $\llbracket|u-\check{a}| \leq \varepsilon \rrbracket>0$, then there exists a $b \in \sigma(A)$ such that $|b-a| \leq \varepsilon$, where $\sigma(A)$ is the spectrum of $A$. This can be seen as follows. $\llbracket|u-\check{a}| \leq \varepsilon \rrbracket>0$ implies that

$$
\llbracket a-\varepsilon \leq u \leq a+\varepsilon \rrbracket>0
$$

Hence we have $E_{a+\varepsilon}-E_{a-\varepsilon-0}>0$. This implies $\exists b \in \sigma(A)(b \in[a-\varepsilon, a+\varepsilon])$.

$\overline{\mathbb{C}}$ is obtained from $\mathbb{C}$ by adding the point at the infinite. Let $f(z)$ be a function from $\overline{\mathbb{C}}$ to $\mathbb{C}$, let $A$ be a normal operator and let $\sigma(A)$ be its spectrum, which may include the point at infinity. If $f(z)$ is holomorphic in a neighborhood of $\sigma(A)$, and if $u$ corresponds to $A$ and $v$ corresponds to $f^{\prime}(A)$, then we can repeat the argument of the last section and prove that

$\llbracket f$ is holomorphic at $u$ and its derivative at $u$ is $v \rrbracket=I$.

That is, $f(z)$ is holomorphic at $A$ and the derivative of $f(z)$ at $A$ is $f^{\prime}(A)$.

\subsection{Convergence}

In this section, we shall consider the relation between the convergence of reals in $V^{(\mathcal{B})}$ and the convergence of the self-adjoint operators.

Theorem 1.6.1. Let $A_{0}, A_{1}, \ldots, A$ be pairwise commutable bounded self-adjoint operators with $M$ a uniform bound of $\left\|A_{0}\right\|,\left\|A_{1}\right\|, \ldots,\|A\|$. Furthermore let $\llbracket u: \omega \rightarrow \mathbb{R} \rrbracket=I$, let $u(\check{i})$ correspond to $A_{i}$ for each $i \in \omega$, and let $v \in \mathbb{R}^{(\mathcal{B})}$ correspond to $A$. If $\llbracket \lim _{n \rightarrow \infty} u(n)=v \rrbracket=I$, then $\lim _{n \rightarrow \infty} A_{n}=A$ in the strong topology.

Proof. From $\llbracket \lim _{n \rightarrow \infty} u(n)=v \rrbracket=I$ it follows that for every $\varepsilon>0$ there exists a partition of unity $\left\{P_{i}\right\}$ and $\left\{n_{i}\right\}$ such that for every $\left\{m_{i}\right\}$ with $\forall_{i}\left(m_{i} \geq n_{i}\right)$

$$
\left\|A-\sum_{i} A_{m_{i}} P_{i}\right\|<\frac{\varepsilon}{2}
$$


Take an arbitrary $x$ with $\|x\|=1$. For every $\varepsilon_{1}>0$, there exists a $k$ such that

$$
\left\|x-\sum_{i=0}^{k} P_{i} x\right\|<\varepsilon_{1} .
$$

Let $N=\max \left(n_{0}, n_{1}, \ldots, n_{k}\right)$ and take any $m \geq N$. Then let $y=A x-$

$$
\begin{aligned}
& \sum_{i=0}^{k} A_{m} P_{i} x-\sum_{k<i} A_{n_{i}} P_{i} x, u=\sum_{k<i} A_{m} P_{i} x \text { and } v=\sum_{k<i} A_{n_{i}} P_{i} x . \\
&\left\|A x-A_{m} x\right\|^{2}=\|y-u+v\|^{2} \\
& \leq\|y\|^{2}+\|u\|^{2}+\|v\|^{2}+2\|y\|\|v\|+2\|y\|\|v\|+2\|u\|\|v\| .
\end{aligned}
$$

Since $\|y\|<\frac{\varepsilon}{2}$, it suffices to show that $\|u\|$ and $\|v\|$ go to 0 if $\varepsilon_{1}$ goes to 0 .

$$
\|u\|=\left\|\sum_{k<i} A_{m} P_{i} x\right\| \leq M\left\|\sum_{k<i} P_{i} x\right\|<\varepsilon_{1} M .
$$

Since $A_{n_{i}}$ and $P_{i}$ are commutable,

$$
\begin{aligned}
\|v\|^{2} & =\left\|\sum_{k<i} A_{n_{i}} P_{i} x\right\|^{2}=\sum_{k<i}\left\|A_{n_{i}} P_{i} x\right\|^{2} \\
& \leq M^{2} \sum_{k<i}\left\|P_{i} x\right\|^{2}=M^{2}\left\|\sum_{k<i} P_{i} x\right\|^{2}<M^{2} \varepsilon_{1}^{2} .
\end{aligned}
$$

Remark. The uniform boundedness of the $\left\|A_{i}\right\|$ in Theorem 1.6.1 is necessary as is easily seen in the following example.

Let $\left\{P_{i}\right\}$ be a partition of unity with $P_{i}>0$ for each $i$. Let $x$ have the property that $\forall i\left(\left\|P_{i} x\right\|>0\right)$; let $a_{i}=1 /\left\|P_{i} x\right\|$ and let $A_{i}=a_{i}^{2} P_{i}$. Then for every $\sum_{j} m_{j} P_{j}^{\prime} \geq \sum_{i}(i+1) P_{i}$

$$
\sum_{j} A_{m_{j}} P_{j}^{\prime}=0
$$

Since $P_{j}^{\prime} \cdot P_{i}>0$ implies $m_{j} \geq i+1$.

Hence $\llbracket \lim _{n \rightarrow \infty} u(n)=0 \rrbracket=I$, where $u(\check{i})$ corresponds to $A_{i}$ for every $i$. However $\left(A_{i} x, x\right)=\left\|a_{i} P_{i} x\right\|^{2}=1$ so $A_{i}$ does not converge to 0 in the weak topology. Also the converse of the theorem is not true as is easily seen from the following example. Consider the Hilbert space $L^{2}[0,1]$ and define $P[\alpha, \beta]$ to be the projection which restricts $x$ to the domain $[\alpha, \beta]$. Put $P_{0}=I, P_{1}=P[0,1 / 2], P_{2}=$ $[1 / 2,1], P_{3}=P[0,1 / 4], P_{4}=P[1 / 4,1 / 2], P_{5}=P[1 / 2,3 / 4], P_{6}=P[3 / 4,1], \ldots$ Then obviously $P_{0}, P_{1}, P_{2}, \ldots \rightarrow 0$ in the strong topology. However it has a subsequence in $V^{(\mathcal{B})} P_{0}, P_{1} \cdot P_{1}+P_{2} \cdot P_{2}, P_{3} \cdot P_{3}+P_{4} \cdot P_{4}+P_{5} \cdot P_{5}+P_{6} \cdot P_{6}, \ldots$ which converges to $I$ in $V^{(\mathcal{B})}$. Therefore it does not converge to 0 in $V^{(\mathcal{B})}$. 
Proposition 1.6.2. Let $A_{0}, A_{1}, \ldots, A$ be pairwise commutable bounded selfadjoint operators and $A_{0}, A_{1}, A_{2}, \ldots \rightarrow A$ uniformly. If $\llbracket u: \omega \rightarrow \mathbb{R} \rrbracket=I$, if $u(\check{i})$ corresponds to $A_{i}$ for each $i \in \omega$ and if $v \in \mathbb{R}^{(\mathcal{B})}$ corresponds to $A$, then

$$
\llbracket \lim _{n \rightarrow \infty} u(n)=v \rrbracket=I .
$$

Proof. Without loss of generality, we assume $A=0$. For every $\varepsilon>0$, take $k$ such that $\forall i \geq k\left(\left\|A_{i}\right\| \leq \varepsilon\right)$. Then

$$
\begin{gathered}
\llbracket \forall i \in \omega(i \geq \check{k} \Rightarrow|u(i)| \leq \check{\varepsilon}) \rrbracket \\
=\inf _{i \geq k} \llbracket|u(\check{i})| \leq \check{\varepsilon} \rrbracket=I .
\end{gathered}
$$

Therefore

$$
\llbracket \lim _{n \rightarrow \infty} u(n)=0 \rrbracket=I
$$

Remark. The converse of Proposition 1.6.2 is also false as is easily seen in the following example. Let $\left\{P_{i}\right\}_{i<\omega}$ be a partition of unity satisfying $\forall i<\omega\left(P_{i}>0\right)$. The sequence $P_{0}, P_{0}+P_{1}, P_{0}+P_{1}+P_{2}, \ldots$ converges to $I$ in $V^{(\mathcal{B})}$ but it does not converge to $I$ uniformly.

Proposition 1.6.3. Let $A$ be a self-adjoint operator and let $f_{0}, f_{1}, \ldots, f$ be Borel functions with $f_{0}, f_{1}, \ldots \rightarrow f$ uniformly on every compact subset of $\mathbb{R}$. Let $\llbracket u: \omega \rightarrow \mathbb{R} \rrbracket=I, v \in \mathbb{R}^{(\mathcal{B})}$ and $u(\check{0}), u(\check{1}), \ldots, v$ correspond to $f_{0}(A), f_{1}(A), \ldots, f(A)$ respectively. Then

$$
\llbracket \lim _{n \rightarrow \infty} u(n)=v \rrbracket=I .
$$

Proof. Without loss of the generality, we assume $f(x)=0$. Let $A=\int \lambda d E_{\lambda}$ and $P_{n}=E_{n}-E_{-n}$. Take any $\varepsilon>0$. Define $n_{i}$ such that

$$
\forall n \geq n_{i} \quad \forall x \in[-i, i] \quad\left|f_{n}(x)\right| \leq \varepsilon .
$$

Let $N=n_{1} P_{1}+n_{2}\left(P_{2}-P_{1}\right)+n_{3}\left(P_{3}-P_{2}\right)+\cdots$. Then for every $\sum_{i} m_{i} P_{i}^{\prime} \geq N$, we have

$$
\sum_{i} f_{m_{i}}(A) P_{i}^{\prime}=\sum_{i j} f_{m_{i}}(A) P_{i}^{\prime}\left(P_{j}-P_{j-1}\right) .
$$

Let $B_{i j}=f_{m_{i}}(A)\left(P_{j}-P_{j-1}\right) P_{i}^{\prime}$. Then $\left\|B_{i j}\right\| \leq \varepsilon$, i.e. $-\varepsilon \leq B_{i j} \leq \varepsilon$. Therefore

$$
-\varepsilon=-\varepsilon \sum_{i j} P_{i}^{\prime}\left(P_{j}-P_{j-1}\right) \leq \sum_{i j} B_{i j} P_{i}^{\prime}\left(P_{j}-P_{j-1}\right) \leq \varepsilon \sum_{i j} P_{i}^{\prime}\left(P_{j}-P_{j-1}\right)=\varepsilon .
$$




\subsection{Semi-group of self-adjoint operators}

In this section, we shall discuss semi-groups of (possibly unbounded) self-adjoint operators. In order to discuss the convergence of unbounded self-adjoint operators, we need several definitions.

Let $P_{1}$ and $P_{2}$ be projections. If $P_{1} \leq P_{2}$, then $\forall x\left(\left\|P_{1} x\right\| \leq\left\|P_{2} x\right\|\right)$. From this it follows that $P_{2} x$ is a better approximation of $x$ than $P_{1} x$ if $P_{1} \leq P_{2}$.

Definition 1.7.1. Let $A=\int \lambda d E_{\lambda}$. Then by a segment projection of $A$, we mean a projection of the form $E_{M}-E_{N}$, where $N \leq M$. For every $x_{1}, \ldots, x_{n}$ and every $\varepsilon>0$, there exists a segment projection $P$ of $A$ such that

$$
\left\|P x_{i}-x_{i}\right\|<\varepsilon \quad \text { for every } i \text {. }
$$

If we choose Ay to be some $x_{i}$, we have the following. For every $x_{1}, \ldots, x_{n}, y_{1}, \ldots, y_{m} \in$ $\mathcal{D}(A)$, and every $\varepsilon>0$, there exists a segment projection $P$ of $A$ such that

$$
\left\|P x_{i}-x_{i}\right\|<\varepsilon \quad \text { for every } i
$$

and

$$
\left\|A P y_{j}-A y_{j}\right\|<\varepsilon \quad \text { for every } j .
$$

We also remark here that $A$ is continuous i.e. bounded on the range of its segment projection.

Lemma 1.7.1. Let $A_{1}, A_{2}, \ldots, B_{1}, \ldots, B_{n}$ be pairwise commutable self-adjoint operators. For every $x$ in $\mathcal{D}\left(B_{1}\right) \cap \cdots \cap \mathcal{D}\left(B_{n}\right)$, and every $\varepsilon>0$ there exists a $y$ in $\bigcap_{i} \mathcal{D}\left(A_{i}\right) \cap \bigcap_{j} \mathcal{D}\left(B_{j}\right)$ such that

$$
\begin{gathered}
\|x-y\|<\varepsilon \text { and } \\
\left\|B_{j} x-B_{j} y\right\|<\varepsilon \quad \text { for every } j .
\end{gathered}
$$

Proof. There exists a segment projection $P_{i}^{\prime}$ of $B_{i}$, for each $i$, such that

$$
\left\|P_{1}^{\prime} \cdots P_{n}^{\prime} x-x\right\|<\frac{\varepsilon}{2}
$$

and $\left\|B_{j} P_{1}^{\prime} \cdots P_{n}^{\prime} x-B_{j} x\right\|<\frac{\varepsilon}{2}$ for every $j$. Let $\mathcal{M}_{i}=\mathcal{R}\left(P_{i}^{\prime}\right)$ and $P_{1}^{\prime} \cdots P_{n}^{\prime} x=$ $x_{0}$. Suppose we have defined $x_{0}, x_{1}, \ldots, x_{i}$ such that $x_{0}, \ldots, x_{i} \in \mathcal{M}_{1} \cap \cdots \cap \mathcal{M}_{n}$

$$
\begin{gathered}
x_{k} \in \mathcal{D}\left(A_{1}\right) \cap \cdots \cap \mathcal{D}\left(A_{k}\right) \cap \mathcal{M}_{1} \cap \cdots \cap \mathcal{M}_{n} \quad(0 \leq k \leq i) \\
\left\|x_{k+1}-x_{k}\right\|<\frac{\varepsilon}{2^{k+2}} \quad(0 \leq k \leq i-1)
\end{gathered}
$$

and

$$
\left\|B_{j} x_{k+1}-B_{j} x_{k}\right\|<\frac{\varepsilon}{2^{k+2}} \text { for every } j \quad(0 \leq k \leq i-1) .
$$


Then we define $x_{i+1}$ as follows.

Since the $B_{j}$ are continuous on $\mathcal{M}_{k}(1 \leq k \leq n)$ and $x_{i} \in \mathcal{M}_{k}$, take a sufficient large segment projection $P_{i+1}$ of $A_{i+1}$ so that

$$
\left\|P_{i+1} x_{i}-x_{i}\right\|<\frac{\varepsilon}{2^{i+2}}
$$

and

$$
\left\|B_{j} P_{i+1} x_{i}-B_{j} x_{i}\right\|<\frac{\varepsilon}{2^{i+2}} \quad \text { for every } j .
$$

If $x_{i+1}=P_{i+1} x_{i}$, then obviously

$$
\begin{gathered}
x_{i+1} \in \mathcal{D}\left(A_{1}\right) \cap \cdots \cap \mathcal{D}\left(A_{i+1}\right), \\
\left\|x_{i+1}-x_{i}\right\|<\frac{\varepsilon}{2^{i+2}},
\end{gathered}
$$

and

$$
\left\|B_{j} x_{i+1}-B_{j} x_{i}\right\|<\frac{\varepsilon}{2^{i+1}} \quad \text { for every } j .
$$

If $y=\lim _{i \rightarrow \infty} x_{i}$, then $\|x-y\|<\varepsilon, y \in \mathcal{R}\left(P_{i}\right) \subseteq \mathcal{D}\left(A_{i}\right), y \in \mathcal{M}_{i}$, and $\left\|B_{j} x-B_{j} y\right\|<$ $\varepsilon$ for every $j$.

Definition 1.7.2. Let $A, A_{1}, A_{2}, \ldots$ be pairwise commutable self-adjoint operators. Then $A_{1}, A_{2}, \ldots \rightarrow A$ in the strong sense iff for every $n$ and every $x \in \mathcal{D}(A) \cap \bigcap_{i \geq n} \mathcal{D}\left(A_{i}\right)$,

$$
A_{n} x, A_{n+1} x, \ldots \rightarrow A x .
$$

If $A, A_{1}, A_{2}, \ldots$ are bounded, then ' $A_{1}, A_{2}, \ldots \rightarrow A$ in the strong sense' is equivalent to ' $A_{1}, A_{2}, \ldots \rightarrow A$ in the strong topology'. If $A, B_{1}, B_{2}, \ldots$ are pairwise commutable self-adjoint operators and $B_{1}, B_{2}, \ldots \rightarrow 0$ in the strong topology, then $A_{1}, A_{2}, \ldots \rightarrow A$ in the strong sense, where $A_{i}=A+B_{i}$.

Lemma 1.7.2. Let $A, B, A_{1}, A_{2}, \ldots$ be pairwise commutable self-adjoint operators satisfying the following conditions:

1) $\forall i \quad A_{i} \leq B$

2) $A_{1}, A_{2}, \ldots \rightarrow A$ in the strong sense.

Then $A \leq B$.

Proof. Let $x \in \mathcal{D}(A) \cap \mathcal{D}(B)$. By Lemma 1.7.1, there exists on $x_{n} \in \mathcal{D}(A) \cap$ $\mathcal{D}(B) \cap \bigcap_{i} \mathcal{D}\left(A_{i}\right)$ such that

1) $\left\|x-x_{n}\right\|<\frac{1}{2^{n}}$

2) $\left\|A x-A x_{n}\right\|<\frac{1}{2^{n}}$ 
3) $\left\|B x-B x_{n}\right\|<\frac{1}{2^{n}}$.

Fix a sequence $x_{1}, x_{2}, \ldots$ satisfying 1$), 2$ ), and 3 ). For every $\varepsilon>0$, there exists an $x_{n}$ such that

$$
\begin{gathered}
(A x, x) \leq\left(A x_{n}, x_{n}\right)+\frac{\varepsilon}{3} \text { and } \\
\left(B x_{n}, x_{n}\right) \leq(B x, x)+\frac{\varepsilon}{3} .
\end{gathered}
$$

Since $A_{1}, A_{2}, \ldots \rightarrow A$ and $x_{n} \in \mathcal{D}(A) \cap \bigcap_{i} \mathcal{D}\left(A_{i}\right)$, there exists an $A_{m}$ such that

$$
\left(A x_{n}, x_{n}\right) \leq\left(A_{m} x_{n}, x_{n}\right)+\frac{\varepsilon}{3} .
$$

Hence we have $(A x, x) \leq(B x, x)+\varepsilon$. Since $\varepsilon$ is an arbitrary positive number, we have

$$
(A x, x) \leq(B x, x) .
$$

Definition 1.7.3. A subset $G$ of $(0, \infty)$ is called a regular semi-group if the following conditions are satisfied.
1) $x, y \in G \Rightarrow x+y \in G$
2) $x, y \in G, x<y \Rightarrow y-x \in G$
3) $x \in G \Rightarrow \frac{x}{2} \in G$.

The following theorem is very easily proved in the classical theory of functional equations.

Theorem 1.7.3 (cf. [1]). Let $G$ be a regular semi-group and let $f: G \rightarrow \mathbb{R}$ satisfy the following conditions:

1) $\forall x, y \in G \quad f(x+y)=f(x) \cdot f(y)$

2) There exists an $a>0$ such that $f$ is bounded from above in $(0, a) \cap G$. $e^{c x}$.

Then either $\forall x \in G f(x)=0$ or there exists a $c \in \mathbb{R}$ such that $\forall x \in G f(x)=$

The following theorem is an interpretation of Theorem 1.7.3.

Theorem 1.7.4. Let $G$ be a regular semi-group and let $\left\{T_{s} \mid s \in G\right\}$ be pairwise commutable self-adjoint operators satisfying the following conditions:

1) $\forall s, t \in G \quad T_{s+t}=T_{s} \cdot T_{t}$.

2) If $s_{1}, s_{2}, \ldots \rightarrow t$ in $G$, then $T_{s_{1}}, T_{s_{2}}, \ldots \rightarrow T_{t}$ in the strong sense. 
Then there exists a projection $P$ and a self-adjoint operator $A$ such that

1) $\forall s \in G \quad T_{s}=P \cdot e^{s A}$

2) $P, A$, and $\left\{T_{s}\right\}$ are pairwise commutable.

Proof. First take any $s_{0} \in G$. Let $B=\max \left(I, T_{s_{0}}\right)$. Then it is very easily seen that

$$
T_{x} \leq B
$$

if $x$ is of the form $\frac{k}{2^{n}} s_{0}$ where $k \leq 2^{n}$. By Lemma 1.7.2,

$$
\forall x \in G \cap\left(0, s_{0}\right) \quad T_{x} \leq B .
$$

Now let $B$ be a complete Boolean algebra such that all $T_{s}$ are in $(\mathcal{B})$. Then $\check{G}$ is a regular semi-group in $V^{(\mathcal{B})}$. By Proposition 1.4.1, there exists an $f \in V^{(\mathcal{B})}$ such that

1) $\llbracket f: \check{G} \rightarrow \mathbb{R} \rrbracket=I$ and

2) $\forall s \in G \llbracket f(\check{s})=u_{s} \rrbracket=I$,

where $u_{s}$ corresponds to $T_{s}$ for every $s \in G$. Let $v \in \mathbb{R}^{(\mathcal{B})}$ correspond to $B$. Then it is easily proved that

$$
\begin{gathered}
\llbracket \forall s, t \in \check{G} \quad f(s+t)=f(s) \cdot f(t) \rrbracket=I, \text { and } \\
\llbracket \forall s \in \check{G} \cap\left(0, \check{s_{0}}\right) f(s) \leq v \rrbracket=I .
\end{gathered}
$$

By Theorem 1.7.3, we have

$$
\llbracket \forall s \in \check{G}(f(s)=0) \text { or } \exists a \in \mathbb{R} \forall s \in \check{G}\left(f(s)=e^{s a}\right) \rrbracket=I .
$$

Also

$$
\llbracket \forall s \in \check{G}(f(s)=0) \rrbracket=\llbracket f\left(\check{s_{0}}\right)=0 \rrbracket
$$

where $s_{0}$ is a member of $G$.

Let $P=\llbracket f\left(\check{s_{0}}\right) \neq 0 \rrbracket$. Then

$$
\begin{aligned}
& I-P=\llbracket \forall s \in \check{G}(f(s)=0) \rrbracket \text { and } \\
& \llbracket \exists a \in \mathbb{R} \forall s \in \check{G}\left(f(s)=e^{s a}\right) \rrbracket=P .
\end{aligned}
$$

Choose $u \in \mathbb{R}^{(\mathcal{B})}$ so that

$$
\llbracket \forall s \in \check{G}\left(f(s)=e^{s u}\right) \rrbracket=P,
$$

and let $A$ correspond to $u$. If $u_{s}=e^{s u} \cdot P+0 \cdot(I-P)$, then $\forall s \in G \llbracket(f(s)=$ $\left.u_{s}\right) \rrbracket=I$, that is,

$$
\forall s \in G T_{s}=P \cdot e^{s A}+0 \cdot(I-P)=P \cdot e^{s A} .
$$




\subsection{Complete Boolean algebras of Banach spaces}

A bounded linear operator $P$ of a Banach space is called a projection if $P^{2}=P$. A Boolean algebra of projections in a Banach space and its completeness are defined in the same way as before.

Definition 1.8.1. Let $\mathcal{B}$ be a complete Boolean algebra. The Boolean algebra $\mathcal{B}$ satisfies the $(\omega, \omega)$-weak distributive law $((\omega, \omega)$-WDL) iff for every family $\left\{P_{n m} \mid n, m \in \omega\right\} \subseteq \mathcal{B}$

$$
\inf _{n<\omega} \sup _{m<\omega} P_{n m}=\sup _{f \in \omega^{\omega}} \inf _{n<\omega} \sup _{m \leq f(n)} P_{n m}
$$

The Boolean algebra $\mathcal{B}$ satisfies the $\left(\omega_{\alpha}, \omega_{\beta}\right)$-WDL iff for every family $\left\{P_{\xi \eta} \mid \xi<\right.$ $\left.\omega_{\alpha} \wedge \eta<\omega_{\beta}\right\} \subseteq \mathcal{B}$,

$$
\inf _{\xi<\omega_{\alpha}} \sup _{\eta<\omega_{\beta}} P_{\xi \eta}=\sup _{f \in \omega_{\beta} \omega_{\alpha}} \inf _{\xi<\omega_{\alpha}} \sup _{\eta<f(\xi)} P_{\xi \eta}
$$

The following proposition is well-known in set theory (cf. Corollary 23. 37. in [10]).

Theorem 1.8.1. The Boolean algebra $\mathcal{B}$ satisfies the $\left(\omega_{\alpha}, \omega_{\beta}\right)$-WDL iff $\llbracket c f\left(\left(\omega_{\beta}\right)^{\sqrt{ }}\right)>$ $\left(\omega_{\alpha}\right) \sqrt{ } \rrbracket=I$ in $V^{(\mathcal{B})}$, where $c f$ is the character of cofinality.

For the meaning of the $(\omega, \omega)$-WDL, see Theorem 20. 4. in [10].

Theorem 1.8.2. Let $\mathcal{B}$ be a complete Boolean algebra of projections in a Banach space. Then $\mathcal{B}$ satisfies the $(\omega, \omega)$-WDL.

Proof. It suffices to show that

$$
\inf _{n<\omega} \sup _{m<\omega} P_{n m} \leq \sup _{f \varepsilon \omega \omega} \inf _{n<\omega} \sup _{m \leq f(n)} P_{n m}
$$

for $\left\{P_{n m} \mid n<\omega \wedge m<\omega\right\} \subseteq \mathcal{B}$.

Take any $P$ in $\mathcal{B}$ for which

1) $0<P \leq \inf _{n<\omega} \sup _{m<\omega} P_{n m}$.

We want to show that

$$
P \cdot \sup _{f \varepsilon \omega^{\omega}} \inf _{n<\omega} \sup _{m \leq f(n)} P_{n m}>0 .
$$

There exists an $x$ such that

$$
\|x\|=1 \wedge x=P x .
$$

By virtue of 1$)$, if $n_{1}$ is sufficiently large, then $x$ and $\left(\sup _{m \leq n_{1}} P_{1 m}\right) \cdot x$ are very close. So there exists an $n_{1}$ such that

$$
\left\|x-x_{1}\right\| \leq \frac{1}{3}
$$


where $x_{1}=\left(\sup _{m \leq n_{1}} P_{1 m}\right) \cdot x$. In the same way, there exist $n_{2}, \ldots, n_{k}$ such that

$$
\left\|x_{i-1}-x_{i}\right\|<\frac{1}{3^{i}} \quad(2 \leq i \leq k)
$$

where $x_{i}=\left(\sup _{m \leq n_{i}} P_{i m}\right) \cdot x_{i-1}$. Let $x_{0}=\lim _{n \rightarrow \infty} x_{n} .\left\|x_{0}\right\|>\frac{1}{2}$. Since $P$ and $P_{n m}$ 's are pairwise commutable, $P \cdot x_{0}=x_{0}$. If $f(k)=n_{k}$, then

$$
\left(\inf _{n<\omega} \sup _{m \leq f(n)} P_{n m}\right) \cdot x_{0}=x_{0} .
$$

Therefore

$$
P \cdot \inf _{n<\omega} \sup _{m \leq f(n)} P_{n m}>0 .
$$

Theorem 1.8.3. Let $\mathcal{B}$ be a complete Boolean algebra of projections in a Banach space and let $\operatorname{cf}\left(\omega_{\beta}\right)>\omega$. Then $\mathcal{B}$ satisfies the $\left(\omega_{\alpha}, \omega_{\beta}\right)$-WDL.

Proof. Let $P$ and $P_{\xi \eta}$ 's be in $\mathcal{B}$. We want to show that for every $P$ with

$$
\begin{gathered}
0<P \leq \inf _{\xi<\omega_{a}} \sup _{\eta<\omega_{\beta}} P_{\xi \eta}, \\
0<P \cdot \sup _{f \in \omega_{\beta} \omega_{a}} \inf _{\xi<\omega_{a}} \sup _{\eta<f(\xi)} P_{\xi \eta} .
\end{gathered}
$$

Let $x \neq 0$ and $P x=x$. Fix $\xi<\omega_{\alpha}$. Since $\left(\sup _{\eta<\omega_{\beta}} P_{\xi \eta}\right) \cdot x=x, x$ is a cluster point of the set $\left\{\left(\sup _{\eta<\theta} P_{\xi \eta}\right) \cdot x \mid \theta<\omega_{\beta}\right\}$. Therefore there exists an $\omega$-sequence

$$
\theta_{0} \leq \theta_{1} \leq \theta_{2} \leq \cdots<\omega_{\beta}
$$

such that $\left(\sup _{\eta<\theta_{i}} P_{\xi \eta}\right) \cdot x \rightarrow x$ if $i \rightarrow \omega$. If $f(\xi)=\sup _{i<\omega} \theta_{i}$, then $f(\xi)<\omega_{\beta}$ and $\left(\sup _{\eta<f(\xi)} P_{\xi \eta}\right)^{\eta<\theta_{i}} \cdot x=x$.

\subsection{Piecewise convergence}

In the work ahead, we need a weaker notion of convergence for possibly unbounded operators, than convergence in the strong sense.

Definition 1.9.1. Let $A, A_{1}, A_{2}, \ldots$ be pairwise commutable normal operators. Then $A_{1}, A_{2}, \ldots \rightarrow A$ piecewise iff there exists a partition $\left\{P_{\alpha}\right\}$ of unity satisfying the following properties:

1) $A P_{\alpha}$ and $A_{i} P_{\alpha}$ are bounded for every $i$ and $\alpha$.

2) For every $\alpha, A_{1} P_{\alpha}, A_{2} P_{\alpha}, \ldots \rightarrow A P_{\alpha}$ in the strong topology. 
Theorem 1.9.1. Let $A, A_{1}, A_{2}, \ldots$ be pairwise commutable and let $A_{1}, A_{2} \rightarrow A$ in the strong sense. Then $A_{1}, A_{2}, \ldots \rightarrow A$ piecewise.

Proof. Let $\mathcal{B}$ be a complete Boolean algebra such that $A, A_{1}, A_{2}, \ldots$ are in $(\mathcal{B})$. Consider the family of all sets $\left\{P_{\alpha}\right\}$ of projections in $\mathcal{B}$ with the following properties:

1) $P_{\alpha}>0$ for every $\alpha$ and $P_{\alpha} \cdot P_{\beta}=0$ for every $\alpha$ and $\beta$ with $\alpha \neq \beta$.

2) For every $\alpha$ and $i, A P_{\alpha}$ and $A_{i} P_{\alpha}$ are bounded and $A_{1} P_{\alpha}, A_{2} P_{\alpha}, \ldots \rightarrow A P_{\alpha}$ in the strong topology.

Let $\mathfrak{F}=\left\{P_{\alpha}\right\}$ be maximal in the family. Define $\tilde{P}$ by the following:

$$
\tilde{P}=\sup \left\{P_{\alpha} \mid P_{\alpha} \in \mathfrak{F}\right\} .
$$

If $\tilde{P}=1$, then the theorem is proved. So assume $I-\tilde{P}>0$. By relativizing everything to $\mathcal{R}(I-\tilde{P})$, we may assume that there are no $P_{\alpha}>0$ satisfying the condition in 2). Let $x \in \mathcal{D}(A) \cap \bigcap_{i} \mathcal{D}\left(A_{i}\right)$ with $x \neq 0$. Let $P, P_{1}, P_{2}, \ldots$ by a sufficiently large segment projection of $A, A_{1}, A_{2}, \ldots$ respectively so that the following conditions are satisfied:

$$
\begin{aligned}
& \|x-P x\|<\varepsilon_{0} \\
& \left\|P x-P_{1} P x\right\|<\varepsilon_{1} \\
& \left\|P_{n+1} P_{n} \cdots P x-P_{n} \cdots P x\right\|<\varepsilon_{n+1} \\
& \lim _{i} \varepsilon_{i}=0
\end{aligned}
$$

and

$$
\sum_{i} \varepsilon_{i}<\|x\|
$$

Let $x_{n}=P_{n}, \ldots, P_{1} P x$, let $x_{0}=\lim _{n \rightarrow \infty} x_{n}$ and let $P_{0}=\inf \left\{P, P_{1}, P_{2}, \ldots\right\}$. Then $x_{0} \neq 0$ and $x_{0} \in \mathcal{R}\left(P_{0}\right)$. So $P_{0}>0$. Obviously $A P_{0}, A_{1} P_{0}, A_{2} P_{0}, \ldots$ are bounded and $A_{1} P_{0}, A_{2} P_{0}, \ldots \rightarrow A P_{0}$ in the strong topology. This is a contradiction.

Remark. It should be noted that a partition of unity $\left\{P_{\alpha}\right\}$ for piecewise convergence in Theorem 1.9.1 can be taken from the smallest complete Boolean algebra generated by projections in $A, A_{1}, A_{2}, \ldots$. The converse of the theorem is false, as is seen from the following example. Let $\left\{P_{i}\right\}$ be a partition of unity, let $P_{i}>0$ for every $i$, and let $x_{i}$ satisfy the conditions $\left\|x_{i}\right\|=1$ and $P_{i} x_{i}=x_{i}$. Define $\lambda_{i j}$ by the following equation

$$
\lambda_{i j}= \begin{cases}2^{2 i-j} & \text { if } i \leq j \\ 0 & \text { otherwise. }\end{cases}
$$


Also define $A_{j}=\sum_{i} \lambda_{i j} P_{i}$. Obviously $A_{j} P_{i}$ is bounded for every $i$ and $j$.

Furthermore $A_{1} P_{i}, A_{2} P_{i}, \ldots \rightarrow 0$ uniformly. If $x=\sum_{i} \frac{1}{2^{i}} x_{i}$, then

$$
A_{j} x=\sum_{i} \frac{\lambda_{i j}}{2^{i}} x_{i}=\sum_{i \leq j} 2^{i-j} x_{i}
$$

So $\left\|A_{j} x\right\| \geq 1$ and $A_{1}, A_{2}, \ldots$ does not converge to 0 in the strong sense. This example shows that the boundedness of $A, A_{1}, A_{2}, \ldots$ does not imply the converse of the theorem.

\subsection{Simultaneous spectrums}

Let $\mathcal{B}_{0}$ and $\mathcal{B}_{1}$ be complete Boolean algebras of projections with $\mathcal{B}_{0} \subseteq \mathcal{B}_{1}$ and let $a, \varepsilon \in \mathbb{R}$, if $u \in \mathbb{R}^{\left(\mathcal{B}_{0}\right)}$ then obviously $u \in \mathbb{R}^{\left(\mathcal{B}_{1}\right)}$. The value of $\llbracket|u-\check{a}|<\check{\varepsilon} \rrbracket$ in $V^{\left(\mathcal{B}_{0}\right)}$ is the same as the value of $\llbracket|u-\check{a}|<\check{\varepsilon} \rrbracket$ in $V^{\left(\mathcal{B}_{1}\right)}$ since only $\mathcal{B}_{0}$ and $V^{\left(\mathcal{B}_{0}\right)}$ are involved in the calculation of $\llbracket|u-\check{a}|<\check{\varepsilon} \rrbracket$.

Definition 1.10.1. Let $A_{1}, \ldots, A_{n}$ be pairwise commutable normal operators and let $a_{1}, \ldots, a_{n} \in \mathbb{C}$. Take a complete Boolean algebra $\mathcal{B}$ such that $A_{1}, \ldots, A_{n}$ are in $(\mathcal{B})$. Let $u_{1}, \ldots, u_{n} \in V^{(\mathcal{B})}$ correspond to $A_{1}, \ldots, A_{n}$ respectively. Then $\left(a_{1}, \ldots, a_{n}\right)$ is a system of simultaneous spectrums for $A_{1}, \ldots, A_{n}$ iff for every $\varepsilon>0$

$$
\llbracket\left|u_{1}-\check{a_{1}}\right|<\check{\varepsilon} \wedge \cdots \wedge\left|u_{n}-\check{a_{n}}\right|<\check{\varepsilon} \rrbracket>0 .
$$

This definition does not depend on the choice of the complete Boolean algebra $\mathcal{B}$. Actually one can always take $\mathcal{B}$ to be the smallest complete Boolean algebra such that $A_{1}, \ldots, A_{n}$ are in $(\mathcal{B})$.

Now we would like to express 'system of simultaneous spectrums' directly without referring to the model. For convenience, we assume that $A_{1}=\int \lambda d E_{\lambda}^{1}, \ldots, A_{n}=$ $\int \lambda d E_{\lambda}^{n}$ are self-adjoint and $a_{1}, \ldots, a_{n}$ are real numbers.

Suppose $\llbracket\left|u_{i}-\check{a}_{i}\right|<\check{\varepsilon} \rrbracket \geq P$, for every $i$ and $P>0$. Then we have

$$
E_{a_{i}-\varepsilon^{\prime}}^{i} \leq I-P \text { and } P \leq E_{a_{i}+\varepsilon^{\prime}}^{i}
$$

where $\varepsilon<\varepsilon^{\prime}$. Therefore we have

$$
E_{a_{i}+\varepsilon^{\prime}}^{i}-E_{a_{i}-\varepsilon^{\prime}}^{i} \geq P .
$$

Then for every $\varepsilon>0$,

$$
\left(E_{a_{1}+\varepsilon}-E_{a_{1}-\varepsilon}\right) \cdots\left(E_{a_{n}+\varepsilon}-E_{a_{n}-\varepsilon}\right)>0 .
$$

On the other hand, suppose this condition is satisfied. For every $\varepsilon$, take $\varepsilon_{0}$ with $0<\varepsilon_{0}<\varepsilon$. Then

$$
\left(E_{a_{1}+\varepsilon_{0}}-E_{a_{1}-\varepsilon_{0}}\right) \cdots\left(E_{a_{n}+\varepsilon_{0}}-E_{a_{1}-\varepsilon_{0}}\right)=P>0 .
$$


Obviously we have that for every $i$

$$
\llbracket\left|u_{i}-\check{a_{i}}\right|<\check{\varepsilon} \rrbracket \geq P .
$$

Therefore $\left(a_{1}, \ldots, a_{n}\right)$ is a system of simultaneous spectrums for $A_{1}, \ldots, A_{n}$. Consequently $\left(a_{1}, \ldots, a_{n}\right)$ is a system of simultaneous spectrums for $A_{1}, \ldots, A_{n}$ iff for every $\varepsilon>0$

$$
\left(E_{a_{1}+\varepsilon}-E_{a_{1}-\varepsilon}\right) \cdots\left(E_{a_{n}+\varepsilon}-E_{a_{n}-\varepsilon}\right)>0 .
$$

If $A_{1}, \ldots, A_{n}$ are normal operators and $a_{1}, \ldots, a_{n}$ are complex numbers, let $A_{j}=B_{j}+i C_{j}$ and $a_{j}=b_{j}+i c_{j}(i=1, \ldots, n)$ where $B_{j}, C_{j}$ are self-adjoint and $b_{j}$ and $c_{j}$ are real numbers. Then $\left(a_{1}, \ldots, a_{n}\right)$ is a system of simultaneous spectrums for $A_{1}, \ldots, A_{n}$ iff $\left(b_{1}, \ldots, b_{n}, c_{1}, \ldots, c_{n}\right)$ is a system of simultaneous spectrums for $B_{1}, \ldots, B_{n}, C_{1}, \ldots, C_{n}$.

If $\left(a_{1}, \ldots, a_{n}\right)$ is a system of simultaneous spectrums for $A_{1}, \ldots, A_{n}$, then $a_{i}$ is a spectrum of $A_{i}$ for every $i$. But the converse is not always true.

Proposition 1.10.1. Let $u_{1}, \ldots, u_{n} \in V^{(\mathcal{B})}$ correspond to normal operators $A_{1}, \ldots, A_{n}$ respectively and $0<P \in \mathcal{B}$. Then there exists a system of simultaneous spectrums $\left(a_{1}, \ldots, a_{n}\right)$ for $A_{1}, \ldots, A_{n}$ such that for every $\varepsilon>0$

$$
\llbracket\left|u_{1}-\check{a_{1}}\right|<\check{\varepsilon} \wedge \cdots \wedge\left|u_{n}-\check{a_{n}}\right|<\check{\varepsilon} \rrbracket \cdot P>0 .
$$

Proof. Without loss of generality, we assume that $A_{1}, \ldots, A_{n}$ are self-adjoint. For every self-adjoint operator $A=\int \lambda d E_{\lambda}$ and every $b \leq c \in \mathbb{R}$ we define $A[b, c]=E_{c}-E_{b-0}$.

Since $P>0$, there exist $i_{1}, \ldots, i_{n} \in \mathbb{Z}$ such that

$$
A_{1}\left[i_{1}, i_{1}+1\right] A_{2}\left[i_{2}, i_{2}+1\right] \cdots A_{n}\left[i_{n}, i_{n+1}\right] \cdot P>0 .
$$

Let $I_{j}=\left[i_{j}, i_{j+1}\right]$ for every $j=1, \ldots, n$. Divide $I_{j}$ into $\left[i_{j}, i_{j}+\frac{1}{2}\right]$ and $\left[i_{j}+\frac{1}{2}, i_{j}+1\right]$, pick one of them and name it $I_{j 1}$ so that

$$
\left(A_{i} I_{11}\right) \cdots\left(A_{n} I_{n 1}\right) \cdot P>0
$$

Repeat this procedure, that is, divide $I_{j k}$ into two half intervals and pick $I_{j k+1}$ among them so that

$$
\left(A_{1} I_{1 k+1}\right) \cdots\left(A_{n} I_{n k+1}\right) \cdot P>0 .
$$

Define $a_{i}$ by $\left\{a_{i}\right\}=\bigcap_{k} I_{i k}$, then $\left(a_{1}, \ldots, a_{n}\right)$ satisfy the proposition.

Let $A_{1}, \ldots, A_{l}$ be pairwise commutable normal operators in $(\mathcal{B})$ and let $f$ be a continuous function. If $f\left(a_{1}, \ldots, a_{l}\right)=0$ for every system of simultaneous 
spectrums of $A_{1}, \ldots, A_{l}$, then $\llbracket f\left(A_{1}, \ldots, A_{l}\right)=\check{0} \rrbracket=\mathbf{I}$. If $f\left(a_{1}, \ldots, a_{l}\right) \neq 0$ for every system of simultaneous spectrums of $A_{1}, \ldots, A_{l}$, then $\llbracket f\left(A_{1}, \ldots, A_{l}\right) \neq$ $\check{0} \rrbracket=I$. In many cases an elementary property of $f\left(A_{1}, \ldots, A_{l}\right)$ in the model holds if the corresponding property of $f\left(a_{1}, \ldots, a_{l}\right)$ holds for every system of simultaneous spectrums of $A_{1}, \ldots, A_{l}$. The converse is not always true. For example let 0 be a continuous spectrum of $A$. Then $\llbracket A \neq \check{0} \rrbracket=I$ though 0 is a spectrum of $A$.

In the following proposition, we prove only one instance of this kind and leave all other cases to the reader.

Proposition 1.10.2. Let $A_{1}, \ldots, A_{l}$ be pairwise commutable normal operators in $(\mathcal{B})$ and let $f$ be a continuous function. If

$$
\forall t \in[0,1] f\left(a_{1}, \ldots, a_{l}, t\right) \neq 0
$$

for every system of simultaneous spectrums of $A_{1}, \ldots, A_{l}$, then

$$
\llbracket \forall t \in[0,1] f\left(A_{1}, \ldots, A_{l}, t\right) \neq \check{0} \rrbracket=I .
$$

Proof. Suppose

$$
\llbracket \exists t \in[0,1] f\left(A_{1}, \ldots, A_{l}, t\right)=\check{0} \rrbracket=P>0 .
$$

Then there exists a normal operator $T$ in $(\mathcal{B})$ such that $0 \leq T \leq 1$ and

$$
\llbracket f\left(A_{1}, \ldots, A_{l}, T\right)=\check{0} \rrbracket=P>0 .
$$

Therefore there exists a system of simultaneous spectrums $\left(a_{1}, \ldots, a_{l}, t\right)$ of $A_{1}, \ldots, A_{l}, T$ such that $0 \leq t \leq 1$ and for every $\varepsilon>0$

$$
\llbracket\left|A_{1}-\check{a_{1}}\right|, \ldots,\left|A_{l}-\check{a_{l}}\right|,|T-\check{t}| \leq \check{\varepsilon} \rrbracket \cdot P>0 .
$$

If $\delta=\left|f\left(a_{1}, \ldots, a_{l}, t\right)\right|>0$, then there exists an $\varepsilon>0$ such that

$\forall x_{1}, \ldots, x_{l}, s\left(\left|x_{1}-a_{1}\right|, \ldots,\left|x_{l}-a_{l}\right|,|s-t| \leq \varepsilon \Rightarrow\left|f\left(x_{1}, \ldots, x_{n}, s\right)-f\left(a_{1}, \ldots, a_{n}, t\right)\right| \leq \frac{\delta}{2}\right)$.

Since $f$ is uniformly continuous in a closed bounded subset of $\mathbb{R}$ in $V^{(\mathcal{B})}$ and $\check{\mathbb{R}}$ is dense in $\mathbb{R}$, this property is extended to $\mathbb{R}$ in $V^{(\mathcal{B})}$.

Then

$$
\begin{gathered}
\llbracket\left|A_{1}-\check{a_{1}}\right|, \ldots,\left|A_{n}-\check{a}_{n}\right|,|T-\check{t}| \leq \check{\varepsilon} \rrbracket \\
\leq \llbracket\left|f\left(A_{1}, \ldots, A_{n}, T\right)-f\left(\check{a_{1}}, \ldots, \check{a_{n}}, \check{t}\right)\right| \leq \frac{\check{\delta}}{2} \rrbracket .
\end{gathered}
$$

Therefore

$$
\begin{aligned}
0 & <P \cdot \llbracket\left|A_{1}-\check{a_{1}}\right|, \ldots,\left|A_{n}-\check{a_{n}}\right|,|T-\check{t}| \leq \check{\varepsilon} \rrbracket \\
& \leq \llbracket f\left(A_{1}, \ldots, A_{n}, T\right) \neq \check{0} \rrbracket \cdot P=0,
\end{aligned}
$$

which is a contradiction. 
Proposition 1.10.3. Let $X$ be a continuous function from $[\check{a}, \check{b}]$ to $\mathbb{C}$ in $V^{(\mathcal{B})}$ and let $M$ be a positive real number such that

$$
\llbracket \forall t \in[\check{a}, \check{b}]|X(t)| \leq \check{M} \rrbracket=I .
$$

We denote the restriction of $X$ to the domain $\{\check{t} \mid t \in[a, b]\}$ by the same $X$ and $X(t) \cdot x$ by $y(t)$ where $x$ is a member of Hilbert space.

$$
\begin{gathered}
\text { If } \llbracket \int_{\breve{a}}^{\breve{b}} X(t) d t=B \rrbracket=I, \text { then } \\
B \cdot x=\int_{a}^{b} y(t) d t .
\end{gathered}
$$

Proof. Since $|X(t)|$ has a uniform bound $\check{M}$ on $[\check{a}, \breve{b}], X(t)$ is continuous with respect to $t \in[a, b]$ in the strong topology. Therefore $y(t)$ is continuous for $t \in[a, b]$ and $\int_{a}^{b} y(t) d t$ is defined. If

$$
B_{n}=\sum_{i=1}^{n} \frac{(\check{b}-\check{a})}{n} \cdot X\left(\check{a}+\frac{i(\check{b}-\check{a})}{n}\right),
$$

then $\llbracket \lim _{n \rightarrow \infty} B_{n}=B \rrbracket=I$, and $\left\|B_{n}\right\|(n=1,2, \ldots)$ and $\|B\|$ have a uniform bound. Therefore

$$
\lim _{n \rightarrow \infty} B_{n} \cdot x=B \cdot x
$$

that is,

$$
\lim _{n \rightarrow \infty} \sum_{i=1}^{n} \frac{(b-a)}{n} y\left(a+\frac{i(b-a)}{n}\right)=B \cdot x .
$$

Hence we have

$$
B \cdot x=\int_{a}^{b} y(t) d t
$$

\subsection{Quantum Logic}

Let $\mathcal{L}$ be the set of all projections in a Hilbert space $\mathcal{H}$ and $\mathcal{M}$ the set of all closed linear spaces in $\mathcal{H}$. The assignment of $\mathcal{R}(P)$ to a projection $P \in \mathcal{L}$ makes a bijection between $\mathcal{L}$ and $\mathcal{M}$. The lattice operations $\wedge$ and $\vee$ are easily defined in $\mathcal{M}$ as follows. Let $M_{i}(i=1,2)$ be a member of $\mathcal{M}$. The closed linear space $M_{1} \wedge M_{2}$ is defined to be $M_{1} \cap M_{2}$ and $M_{1} \vee M_{2}$ is defined to be the least closed linear space including $M_{1} \cup M_{2}$. The bijection between $\mathcal{L}$ and $\mathcal{M}$ introduces the lattice operations $\wedge$ and $\vee$ in $\mathcal{L}$. The new operations $\wedge$ and $\vee$ coincide with the old operations $\wedge$ and $\vee$ when they are applied to commutable projections $P_{1}$ and $P_{2}$. Let $\mathcal{R}(P)=M$. The closed linear space $\mathcal{R}(\neg P)$ is the closed linear 
space of all elements of $\mathcal{H}$ which is orthogonal to every member of $M$. The space $\mathcal{R}(\neg P)$ is denoted by $M^{\perp}$. In another word, the operation $\neg$ in $\mathcal{L}$ corresponds to the operation $\perp$ in $\mathcal{M}$.

$\mathcal{L}$ is an orthomodular lattice (see [2]), that is, a lattice which satisfies the following conditions.

1) $\neg \neg P=P$

2) $P \wedge \neg P=0$ and $P \vee \neg P=I$

3) $\neg\left(P_{1} \wedge P_{2}\right)=\neg P_{1} \vee \neg P_{2}$ and $\neg\left(P_{1} \vee P_{2}\right)=\neg P_{1} \wedge \neg P_{2}$

4) $P_{1} \wedge\left(\neg P_{1} \vee\left(P_{1} \wedge P_{2}\right)\right)=P_{1} \wedge P_{2}$.

$\mathcal{L}$ is also complete as is easily seen from the completeness of $\mathcal{M}$.

Let $P_{1}$ and $P_{2}$ be members of $\mathcal{L}$. The commutativity of $P_{1}$ and $P_{2}$ can be expressed in the following way by the language of $\mathcal{L}$. $P_{1}$ and $P_{2}$ are commutable iff $P_{1}=\left(P_{1} \wedge P_{2}\right) \vee\left(P_{1} \wedge \neg P_{2}\right)$.

The complete orthomodular lattice $\mathcal{L}$ of all projections in $\mathcal{H}$ plays an important role in quantum mechanics (see [2], [3], [5], [6], [11]) and is called a quantum logic.

Now define $V^{(\mathcal{L})}$ in the same way with $V^{(\mathcal{B})}$ as follows:

1) $V_{0}^{(\mathcal{L})}=\phi$

2) if $\alpha$ is a limit, then $V_{\alpha}^{(\mathcal{L})}=\bigcup_{\xi<\alpha} V_{\xi}^{(\mathcal{L})}$

3) $V_{\alpha+1}^{(\mathcal{L})}=\left\{u \mid u: \mathcal{D}(u) \rightarrow \mathcal{L}\right.$ and $\left.\mathcal{D}(u) \subseteq V_{\alpha}^{(\mathcal{L})}\right\}$

and

4) $V^{(\mathcal{L})}=\bigcup_{\alpha \in \mathrm{On}} V_{\alpha}^{(\mathcal{L})}$.

Our intention is to define $\llbracket u \in v \rrbracket$ and $\llbracket u=v \rrbracket$ as before and then to make the following definitions.

1. $\llbracket \neg \varphi \rrbracket=\neg \llbracket \varphi \rrbracket$

2. $\llbracket \varphi_{1} \vee \varphi_{2} \rrbracket=\llbracket \varphi_{1} \rrbracket \vee \llbracket \varphi_{2} \rrbracket$

3. $\llbracket \varphi_{1} \wedge \varphi_{2} \rrbracket=\llbracket \varphi_{1} \rrbracket \wedge \llbracket \varphi_{2} \rrbracket$

4. $\llbracket \forall x \varphi(x) \rrbracket=\inf _{u \in V^{(\mathcal{L})}} \llbracket \varphi(u) \rrbracket$

5. $\llbracket \exists \varphi(x) \rrbracket=\sup _{u \in V^{(\mathcal{L})}} \llbracket \varphi(u) \rrbracket$. 
The difficulty of this program is that the definitions of $\llbracket u \in v \rrbracket$ and $\llbracket u=v \rrbracket$ heavily depend on the implication $\Rightarrow$ and there are no good implication in $\mathcal{L}$. In order to see this, we first define $P_{1} \stackrel{\circ}{\Rightarrow} P_{2}$ to be $\neg P_{1} \vee P_{2}$. The connective $\stackrel{\circ}{\Rightarrow}$ is far from implication. Clearly $\left(P_{1} \stackrel{\circ}{\Rightarrow} P_{2}\right)=I$ is not equivalent to $P_{1} \leq P_{2}$ where $\leq$ is the natural order in the sense of lattice. Almost all properties of implication are violated by $\stackrel{\circ}{\Rightarrow}$. For example, $P_{1} \wedge\left(P_{1} \stackrel{\circ}{\Rightarrow} P_{2}\right) \leq P_{2}$ not always holds.

Now we define $P_{1} \Rightarrow P_{2}$ to be $\neg P_{1} \vee\left(P_{1} \wedge P_{2}\right)$ and $P_{1} \Leftrightarrow P_{2}$ to be $\left(P_{1} \Rightarrow P_{2}\right) \wedge$ $\left(P_{2} \Rightarrow P_{1}\right)$. Then $\left(P_{1} \Rightarrow P_{2}\right)=I$ is equivalent to $P_{1} \leq P_{2}$ and $\left(P_{1} \Leftrightarrow P_{2}\right)=I$ is equivalent to $P_{1}=P_{2}$. Remark that $\left(P_{1} \Rightarrow P_{2}\right)=I$ may be read as $P_{1} \Rightarrow P_{2}$ holds. We also define $P_{1} \perp P_{2}$ to be $P_{1} \leq \neg P_{2}$.

Lemma 1.11.1. Let $P_{\alpha}, Q_{\alpha}, L_{\alpha}(\alpha \in I)$ be members of $\mathcal{L}$ and $L_{\alpha} \perp L_{\beta}$ if $\alpha \neq \beta$. If $\forall \alpha\left(P_{\alpha} \leq L_{\alpha} \wedge Q_{\alpha} \leq L_{\alpha}\right)$, then

$$
\left(\sup _{\alpha} P_{\alpha}\right) \wedge\left(\sup _{\alpha} Q_{\alpha}\right)=\sup _{\alpha}\left(P_{\alpha} \wedge Q_{\alpha}\right) .
$$

Proof. It suffices to show

$$
\left(\sup _{\alpha} P_{\alpha}\right) \wedge\left(\sup _{\alpha} Q_{\alpha}\right) \leq \sup _{\alpha}\left(P_{\alpha} \wedge Q_{\alpha}\right) .
$$

Let $x \in \mathcal{R}\left(\sup _{\alpha} P_{\alpha}\right) \cap \mathcal{R}\left(\sup _{\alpha} Q_{\alpha}\right)$. Then $L_{\alpha} x \in \mathcal{R}\left(P_{\alpha}\right) \cap \mathcal{R}\left(Q_{\alpha}\right)=\mathcal{R}\left(P_{\alpha} \wedge Q_{\alpha}\right)$. Obviously $x=\sum_{\alpha} L_{\alpha} x \in \mathcal{R}\left(\sup _{\alpha}\left(P_{\alpha} \wedge Q_{\alpha}\right)\right)$.

Corollary 1.11.2.

$$
(P \Leftrightarrow Q)=(\neg P \wedge \neg Q) \vee(P \wedge Q)
$$

Proof. $P \Leftrightarrow Q$ is $(\neg P \vee(P \wedge Q)) \wedge(\neg Q \vee(P \wedge Q))$. Define $L_{1}=P \wedge Q$, $L_{2}=\neg(P \wedge Q) . \quad P_{1}=P \wedge Q=Q_{1}, P_{2}=\neg P$ and $Q_{2}=\neg Q$ and use the lemma.

\section{Corollary 1.11.3.}

$$
P \wedge(P \Rightarrow Q) \leq Q .
$$

Remark that this is read as ' $P \wedge(P \Rightarrow Q) \Rightarrow Q$ is true'.

Proof. By using Lemma 1.11.1, we have $P \wedge(\neg P \vee(P \wedge Q))=(0 \vee P) \wedge(\neg P \vee$ $(P \wedge Q))=(0 \wedge \neg P) \vee(P \wedge(P \wedge Q))=P \wedge Q \leq Q$.

Corollary 1.11.3 implies that $\left(P_{1} \Rightarrow P_{2}\right) \wedge\left(P_{1} \wedge Q\right) \Rightarrow\left(P_{2} \wedge Q\right)$ is true.

Corollary 1.11.4.

$$
(P \Rightarrow Q) \wedge \neg Q \leq \neg P .
$$

Proof. By using Lemma 1.11.1 we have $(\neg P \vee(P \wedge Q)) \wedge(\neg Q \vee 0)=\neg P \wedge \neg Q \leq$ $\neg P$. 
The analogy between implication and $\Rightarrow$ seems to stop here. Now we show the inadequacy of $\Rightarrow$ for implication.

Proposition 1.11.5. There is a counter example of

$$
\left(P_{1} \Leftrightarrow P_{2}\right) \leq\left(Q \wedge P_{1} \Rightarrow Q \wedge P_{2}\right) .
$$

Proof. We construct a counterexample in a 3 dimensional Euclidean space. Let $P_{1}$ and $P_{2}$ be commutable and $\mathcal{R}\left(P_{1}\right)$ and $\mathcal{R}\left(P_{2}\right)$ be 2 dimensional and $\mathcal{R}\left(P_{1} \wedge P_{2}\right)$ be 1 dimensional. Let $\mathcal{R}(Q)$ be an 1 dimensional subspace of $\mathcal{R}\left(P_{1}\right)$ such that $Q$ and $P_{1} \wedge P_{2}$ are not commutable. Then we have $\left(Q \wedge P_{1} \Rightarrow Q \wedge P_{2}\right)=(Q \Rightarrow$ $0)=\neg Q$. However $\left(P_{1} \Leftrightarrow P_{2}\right)=P_{1} \wedge P_{2} \not \leq \neg Q$.

Proposition 1.11.5 and $\left(P_{1} \Rightarrow P_{2}\right) \wedge\left(P_{1} \wedge Q\right) \leq\left(P_{2} \wedge Q\right)$ implied by Corollary 1.11.3 give an example such that $P_{1} \wedge P_{2} \leq Q$ does not imply $P_{1} \leq\left(P_{2} \Rightarrow Q\right)$. In order to see this, replace $P_{1}, P_{2}$, and $Q$ by $P_{1} \Leftrightarrow P_{2}, Q \wedge P_{1}$ and $Q \wedge P_{2}$ respectively.

Proposition 1.11.6. There is a counterexample of

$$
\left(P_{1} \Leftrightarrow P_{2}\right) \wedge\left(P_{1} \vee Q\right) \leq\left(P_{2} \vee Q\right)
$$

Proof. We also construct a counterexample in a 3 dimensional Euclidean space. Let $\mathcal{R}\left(P_{1}\right)$ be a 2 dimensional linear space and $\mathcal{R}\left(P_{2}\right)$ be an 1 dimensional subspace of $\mathcal{R}\left(P_{1}\right)$. Then $\mathcal{R}\left(P_{1} \Leftrightarrow P_{2}\right)$ is a 2 dimensional space such that $\mathcal{R}\left(P_{1}\right) \cap \mathcal{R}\left(P_{1} \Leftrightarrow P_{2}\right)=\mathcal{R}\left(P_{2}\right)$. Let $\mathcal{R}(Q)$ be a 2 dimensional space such that $\mathcal{R}\left(P_{1}\right) \cap \mathcal{R}(Q)=\mathcal{R}\left(P_{2}\right)$ and $P_{1}$ and $Q$ are not commutable. Then $P_{1} \vee Q=I$ and $\left(P_{1} \Leftrightarrow P_{2}\right) \wedge\left(P_{1} \vee Q\right)=P_{1} \Leftrightarrow P_{2}$ and $P_{2} \vee Q=Q$. ( $\left(P_{1} \Leftrightarrow P_{2}\right) \not \leq Q$ is obvious whence follows the proposition.

Now we define $\llbracket u \in v \rrbracket$ and $\llbracket u=v \rrbracket$ by the following formula as before.

$$
\begin{aligned}
\llbracket u \in v \rrbracket & =\sup _{y \in \mathcal{D}(u)}(v(y) \wedge \llbracket u=y \rrbracket) \\
\llbracket u=v \rrbracket & =\inf _{x \in \mathcal{D}(u)}(u(x) \Rightarrow \llbracket x \in v \rrbracket) \\
& \wedge \inf _{y \in \mathcal{D}(v)}(v(y) \Rightarrow \llbracket y \in u \rrbracket) .
\end{aligned}
$$

Then an above counterexample is easily transformed into a counterexample of the equality axiom

$$
u=v \Rightarrow(u \in w \Rightarrow v \in w) .
$$

In order to show this, define 0 and 1 as before and let $P_{1}, P_{2}, Q$ not satisfy $\left(P_{1} \Leftrightarrow P_{2}\right) \leq\left(Q \wedge P_{1} \Rightarrow Q \wedge P_{2}\right)$ as in Proposition 1.11.5. Define $u, v$ and $w$ by the following conditions

$$
\begin{aligned}
& \mathcal{D}(u)=\{0\} \quad \text { and } \quad u(0)=P_{1} \text {. } \\
& \mathcal{D}(v)=\{0\} \quad \text { and } \quad v(0)=P_{2} \text {. } \\
& \mathcal{D}(w)=\{1\} \quad \text { and } \quad w(1)=Q \text {. }
\end{aligned}
$$


Then we have the following.

$$
\begin{aligned}
\llbracket 0 \in u \rrbracket & =\sup _{x \in \mathcal{D}(u)}(u(x) \wedge \llbracket 0=x \rrbracket)=P_{1} . \\
\llbracket 0 \in v \rrbracket & =\sup _{x \in \mathcal{D}(v)}(v(x) \wedge \llbracket 0=x \rrbracket)=P_{2} . \\
\llbracket u=v \rrbracket & =\inf _{x \in \mathcal{D}(u)}(u(x) \Rightarrow \llbracket x \in v \rrbracket) \wedge \inf _{x \in \mathcal{D}(v)}(v(x) \Rightarrow \llbracket x \in u \rrbracket) \\
& =\left(P_{1} \Rightarrow P_{2}\right) \wedge\left(P_{2} \Rightarrow P_{1}\right)=\left(P_{1} \Leftrightarrow P_{2}\right) . \\
\llbracket u \in w \rrbracket & =\sup _{x \in \mathcal{D}(w)}(w(x) \wedge \llbracket x=u \rrbracket)=Q \wedge \llbracket 1=u \rrbracket . \\
\llbracket 1=u \rrbracket & =(I \Rightarrow \llbracket 0 \in u \rrbracket) \wedge(u(0) \Rightarrow \llbracket 0 \in 1 \rrbracket) \\
& =\left(I \Rightarrow P_{1}\right)=P_{1} .
\end{aligned}
$$

Clearly $\llbracket u=v \Rightarrow(u \in w \Rightarrow v \in w) \rrbracket=I$ is $\left(P_{1} \Leftrightarrow P_{2}\right) \leq\left(Q \wedge P_{1} \Rightarrow Q \wedge P_{2}\right)$.

Since the notion 'set' strongly depends on equality, this example shows that we cannot construct any kind of set theory on $V^{(\mathcal{L})}$ in a usual sense. Nevertheless $V^{(\mathcal{L})}$ is a nice ground where we observe all Boolean valued models $V^{(\mathcal{B})}$ in $V^{(\mathcal{L})}$ and their mutual relation. For example, suppose that $\mathcal{B}$ and $\mathcal{B}_{0}$ are two complete Boolean algebras in $\mathcal{L}$ and $A \in \mathbb{R}^{(\mathcal{B})}$. If $A$ is not commutable with some member of $\mathcal{B}_{0}$, then $\mathcal{B}_{\alpha}=e^{i \alpha A} \mathcal{B}_{0} e^{-i \alpha A}$ is isomorphic to $\mathcal{B}_{0}$ but different from $\mathcal{B}_{0}$ for every $\alpha \in \mathbb{R}$. These isomorphisms induce isomorphisms between $V^{\left(\mathcal{B}_{0}\right)}$ and $V^{\left(\mathcal{B}_{\alpha}\right)}$. An interesting special case is the case that $\mathcal{B}_{\alpha}=\mathcal{B}_{0}$ for every $\alpha \in \mathbb{R}$. Then we have a continuous nontrivial automorphisms of $V^{\left(\mathcal{B}_{0}\right)}$. The most interesting case of this type appears when a topological group and its representation in the automorphism group of $L$ are given. In this case, there are many isomorphisms and automorphisms of Boolean valued models in $V^{(\mathcal{L})}$. Effective uses of the relation between these isomorphisms and the notion of sets in $V^{(\mathcal{L})}$ seem to the author one of the most interesting subjects in the area. 


\section{Chapter 2}

\section{Boolean Valued Analysis Using Measure Algebras}

As we pointed out earlier, Dana Scott started Boolean valued analysis [4]. He proposed using measure algebras. In this chapter we will study Boolean valued analysis using measure algebras.

\subsection{Measure algebras}

Let $(X, \mathcal{S}, \mu)$ be a measure space, that is, let $\mathcal{S}$ be a Borel field of subsets of $X$, and let $\mu: \mathcal{S} \rightarrow[0, \infty]$ be a $\sigma$-finite $\sigma$-additive measure. (By $\sigma$-finite, we mean that there exists a sequence $X_{1}, X_{2}, X_{3}, \ldots \in \mathcal{S}$ such that for every $n \mu\left(X_{n}\right)<\infty$ and $X=\bigcup_{n} X_{n}$.)

Let $\mathcal{T}=\{B \in \mathcal{S} \mid \mu(B)=0\}$ and $\mathcal{B}=\mathcal{S} / \mathcal{T}$. Then $\mathcal{T}$ is a $\sigma$-additive ideal of $\mathcal{S}$ and $\mathcal{B}$ is a Boolean $\sigma$-algebra. The 0 of $\mathcal{B}$ is represented by the empty set $\phi$ and the $I$ of $\mathcal{B}$ is represented by $X$.

Let $b_{1}=S_{1} / \mathcal{T}$ and $b_{2}=S_{2} / \mathcal{T}$. Then $B_{1}=B_{2}$ means that $S_{1}$ is equal to $S_{2}$ except for a set of measure zero, that is, $S_{1}$ and $S_{2}$ are almost equal. The operations $b_{1}+b_{2}, b_{1} \cdot b_{2}$, and $-b_{1}$ are represented by $S_{1} \cup S_{2}, S_{1} \cap S_{2}$, and $X-S_{1}$ respectively. Furthermore if $b_{i}=S_{i} / \mathcal{T}(i=1,2,3, \ldots)$, then $\sup _{i} b_{i}$ and $\inf _{i} b_{i}$ are represented by $\bigcup_{i} S_{i}$ and $\bigcap_{i} S_{i}$ respectively.

Proposition 2.1.1. The Boolean algebra $\mathcal{B}$ satisfies the countable chain condition (denoted by c.c.c.), i.e. if $\left\{b_{\alpha} \mid \alpha \in J\right\} \subseteq \mathcal{B}$, if $\forall \alpha \in J\left(b_{\alpha} \neq 0\right)$ and $\forall \alpha, \beta \in J\left(\alpha \neq \beta \rightarrow b_{\alpha} \cdot b_{\beta}=0\right)$, then the cardinality of $\left\{b_{\alpha} \mid \alpha \in J\right\}$ is countable.

Proof. Let $b_{\alpha}=S_{\alpha} / \mathcal{T}$. Then $\mu\left(S_{\alpha}\right)>0$ and $\mu\left(S_{\alpha} \cap S_{\beta}\right)=0$ for $\alpha \neq \beta$. Let $X=\bigcup_{n} X_{n}$ such that $\mu\left(X_{n}\right)<\infty$. For every natural number $k$, there are only 
finitely many $S_{\alpha}$ such that

$$
\mu\left(X_{n} \cap S_{\alpha}\right) \geq \frac{1}{k}
$$

since $\mu\left(X_{n}\right) \geq \mu\left(X_{n} \cap\left(S_{\alpha_{1}} \cup \cdots \cup S_{\alpha_{m}}\right)\right) \geq \frac{m}{k}$ if $\mu\left(X_{n} \cap S_{\alpha_{i}}\right) \geq \frac{1}{k}$ and $\alpha_{1}, \ldots, \alpha_{m}$ are distinct. Furthermore $\mu\left(S_{\alpha}\right)>0$ implies that $\exists n \mu\left(S_{\alpha} \cap X_{n}\right)>0$ and that $\alpha$ occurs among finitely many $\alpha$ with $\mu\left(S_{\alpha} \cap X_{n}\right)>0$ for some $n$. The cardinality of all such $\alpha$ is countable.

Since a Boolean $\sigma$-algebra satisfying the c.c.c. is complete (cf. Theorem 3. 28. in $[10]), \mathcal{B}$ is a complete Boolean algebra. Note that $\sup b_{\alpha}$ may not be represented by $\bigcup_{\alpha} S_{\alpha}$ unless $\left\{b_{\alpha} \mid \alpha \in J\right\}$ is countable, where $b_{\alpha}=S_{\alpha} / \mathcal{T}$.

As before we construct a model $V^{(\mathcal{B})}$ using the measure algebra $\mathcal{B}$. As in $\S 2$., the natural numbers in $V^{(\mathcal{B})}$ are of the form $\sum_{\alpha} n_{\alpha} b_{\alpha}$, and the rational numbers in $V^{(\mathcal{B})}$ are of the form $\sum_{\alpha} r_{\alpha} b_{\alpha}$, where $n_{\alpha} \in \omega, r_{\alpha} \in \mathbb{Q}$ and $\left\{b_{\alpha}\right\}$ is a partition of unity.

Let $\left\{b_{\alpha} \mid \alpha \in J\right\}$ be a partition of unity. Since $\mathcal{B}$ satisfies the c.c.c., the cardinality of $\left\{B_{\alpha} \mid \alpha \in I\right\}$ is countable. Let $b_{\alpha}=S_{\alpha} / \mathcal{T}$. Without loss of generality, we can assume that

$$
S_{\alpha} \cap S_{\beta}=\phi \quad \text { if } \alpha \neq \beta
$$

and

$$
\bigcup_{\alpha \in J} S_{\alpha}=X
$$

Therefore a partition of unity is nothing but a partition of $X$ into countable members of $\mathcal{S}$. In this way, $\sum_{\alpha} n_{\alpha} b_{\alpha}$ can be identified with a step function which takes the value $n_{\alpha}$ on each $S_{\alpha}$.

\subsection{Real numbers in the model}

In $\S 1.3$, we defined a real number to be the upper half line of a Dedekind cut. In the case that $\mathcal{B}$ is a measure algebra it is more convenient to define a real number to be the lower half line without the end point, of a Dedekind cut. Therefore the definition of 'a is a real number' is now

$a \subseteq \mathbb{Q} \wedge \exists s \in \mathbb{Q}(s \in a) \wedge \exists s \in \mathbb{Q}(s \notin a) \wedge \forall s \in \mathbb{Q}(s \in a \Leftrightarrow \exists t \in \mathbb{Q}(s<t \wedge t \in a))$.

As in $\S 1.3$,

$$
\mathbb{R}^{(\mathcal{B})}=\left\{u \in V^{(\mathcal{B})} \mid \llbracket u \text { is a real } \rrbracket=I\right\} .
$$


A real in the model is represented by a member of $\mathbb{R}^{(\mathcal{B})}$. For each $u \in \mathbb{R}^{(\mathcal{B})}$ and $r \in \mathbb{Q}$, let

$$
b_{r}=\llbracket \check{r} \in u \rrbracket .
$$

The definition of the real numbers immediately implies the following three conditions.

1) $\inf _{r \in \mathbb{Q}} b_{r}=0$

2) $\sup _{r \in \mathbb{Q}} b_{r}=I$

3) $b_{r}=\sup _{r<s} b_{s}$.

Conversely $\left\{b_{r} \mid r \in \mathbb{Q}\right\}$ satisfying 1$\left.\left.), 2\right), 3\right)$ determines uniquely a $u \in \mathbb{R}^{(s)}$ such that

$$
\forall r \in \mathbb{Q} \quad b_{r}=\llbracket \check{r} \in u \rrbracket .
$$

The meaning of uniqueness is the same as before, that is, if $u_{1}$ and $u_{2}$ satisfy the condition, then

$$
\llbracket u_{1}=u_{2} \rrbracket=I .
$$

Now let $u \in \mathbb{R}^{(\mathcal{B})}$ and $b_{r}=\llbracket \check{r} \in u \rrbracket$. If $b_{r}=S_{r} / \mathcal{T}$, then since $\left\{b_{r} \mid r \in \mathbb{Q}\right\}$ satisfies the above three conditions, we may assume the following without loss of generality
1) $\bigcap_{r \in \mathbb{Q}} S_{r}=\phi$
2) $\bigcup_{r \in \mathbb{Q}} S_{r}=X$
3) $S_{r}=\bigcup_{r<s} S_{s}$.

We define $f: X \rightarrow \mathbb{R}$ by the following condition

$$
f(x)=\sup \left\{r \mid x \in S_{r}\right\} .
$$

It is easily seen that $f$ is a measurable function from $X$ into $\mathbb{R}$ and

$$
S_{r}=\{x \in X \mid r<f(x)\} .
$$

On the other hand, let $f$ be a measurable function from $X$ into $\mathbb{R}$. Let

$$
b_{r}=\{x \in X \mid r<f(x)\} / \mathcal{T}
$$

Then $\left\{b_{r} \mid r \in \mathbb{Q}\right\}$ satisfies the above three conditions and it determines a member of $\mathbb{R}^{(\mathcal{B})}$. This correspondence between $\mathbb{R}^{(\mathcal{B})}$ and the set of all measurable functions is one-to-one in the following sense. If $f$ and $g$ correspond to the same $u \in \mathbb{R}^{(\mathcal{B})}$, then $f$ and $g$ are equal almost everywhere i.e. $\{x \in X \mid f(x) \neq g(x)\}$ 
has measure zero. If $u, v \in \mathbb{R}^{(\mathcal{B})}$ correspond to the same measurable function $f$, then

$$
\llbracket u=v \rrbracket=I .
$$

Let $u, v \in \mathbb{R}^{(\mathcal{B})}$ and $f$ and $g$ be measurable functions corresponding to $u$ and $v$ respectively. Then it is easily seen that

$$
\begin{gathered}
f \pm g \text { corresponds to } u \pm v, \\
f \cdot g \text { corresponds to } u \cdot v,
\end{gathered}
$$

' $f(x)=g(x)$ almost everywhere' is equivalent to

$$
\llbracket u=v \rrbracket=I
$$

and ' $f(x)<g(x)$ almost everywhere' is equivalent to

$$
\llbracket u<v \rrbracket=I .
$$

Let $\lambda \in \mathbb{R}$. Then the constant function, whose value is $\lambda$, corresponds to $\check{\lambda}$.

Proposition 2.2.1. Let $\llbracket u: \omega \rightarrow \mathbb{R} \rrbracket=I$ and $\llbracket u_{i}=u(\check{i}) \rrbracket=I$ for every $i<\omega$. Furthermore let $f_{j}$ correspond to $u_{i}$ for every $i \in \omega$ and let $g$ correspond to $v$. Then ' $f_{i}(x)$ converges to $g(x)$ almost everywhere' is equivalent to

$$
\llbracket \lim _{i \rightarrow \infty} u(i)=v \rrbracket=I .
$$

Proof.

$$
\begin{array}{lll}
\llbracket \lim _{i \rightarrow \infty} u(i)=v \rrbracket=I \quad & \text { iff } & \llbracket \forall \varepsilon>0 \exists n \forall i \geq n|v-u(i)|<\varepsilon \rrbracket=I \\
& \text { iff } \quad \inf _{\varepsilon>0} \sup _{n} \inf _{i \geq n} \llbracket|v-u(i)|<\check{\varepsilon} \rrbracket=I \\
& \text { iff } \quad \inf _{\varepsilon>0} \sup _{n} \inf _{i \geq n} \llbracket\left|v-u_{i}\right|<\check{\varepsilon} \rrbracket=I \\
\text { iff } & \left.\quad \inf _{\varepsilon>0} \operatorname{supinf}_{n}\left\{x|| g(x)-f_{i}(x) \mid<\varepsilon\right\}\right) / \mathcal{T}=X / \mathcal{T} \\
\text { iff } & \left\{x \mid \forall \varepsilon>0 \exists n \forall i \geq n\left(\left|g(n)-f_{i}(n)\right|<\varepsilon\right)\right\} / \mathcal{T}=X / \mathcal{T} \\
\text { iff } & ' f_{i}(x) \text { goes to } g(x) \text { almost everywhere'. }
\end{array}
$$

A non-empty set of real numbers has a least upper bound if it has an upper bound. As an interpretation of this fact, we get the following theorem immediately.

Proposition 2.2.2. Let $M$ be a non-empty set of measurable functions and let $f$ be a measurable function such that

$$
\forall g \in M(g(x) \leq f(x) \text { almost everywhere }) .
$$

Then there exists a measurable function $h$ satisfying the following conditions. 
1. $\forall g \in M(g(x) \leq h(x)$ almost everywhere $)$.

2. If $k$ is a measurable function such that

$$
\forall g \in M(g(x) \leq k(x) \text { almost everywhere }),
$$

then

$$
h(x) \leq k(x) \text { almost everywhere. }
$$

Let $\llbracket u: \omega \rightarrow \mathbb{R} \rrbracket=I$ and let $f_{i}$ correspond to $u(i)$ for $i=0,1,2, \ldots$. Let $\sum_{i} n_{i} b_{i}$ be a natural number in $V^{(\mathcal{B})}$, that is, $n_{i} \in \omega$ and $\left\{b_{i}\right\}_{i}$ be a partition of unity. Let $b_{i}=S_{i} / \mathcal{T}$ and $\left\{S_{i}\right\}_{i}$ satisfy the following conditions.

1. $\bigcup_{i} S_{i}=X$ and

2. $S_{i} \cap S_{j}=\phi$ for $i \neq j$.

Then a measurable function which corresponds to $u\left(\sum_{i} n_{i} b_{i}\right)$ is the function which takes the same value as $f_{n_{i}}$ on $S_{i}$ i.e. the following function $g$,

$$
g(x)=f_{n_{i}}(x) \text { if } x \in S_{i} .
$$

Now we get the following theorem as an interpretation of Bolzano-Weierstrass Theorem.

Proposition 2.2.3. Let $g, f_{0}, f_{1}, f_{2}, \ldots$ be measurable functions such that $\left|f_{i}(x)\right| \leq$ $g(x)$ almost everywhere, for every $i$. Then there exists a measurable function $h(x)$ satisfying the following conditions.

1) $|h(x)| \leq g(x)$ almost everywhere.

2) The function $h$ is a Boolean valued cluster function of $f_{0}, f_{1}, f_{2}, \ldots$ in the following sense. For every $\varepsilon>0$ and for every natural number $m$ there exist a measurable function $k$, a sequence $\left(n_{i}\right)_{i}$ of natural numbers, and a sequence $\left(S_{i}\right)_{i}$ of members of $\mathcal{S}$ such that

a) $m \leq n_{i}$ for every $i$

b) $\bigcup_{i} S_{i}=X$

c) $S_{i} \cap S_{j}=\phi$ for $i \neq j$

d) $k(x)=f_{n_{i}}(x)$ if $x \in S_{i}$

e) $|k(x)-h(x)|<\varepsilon$ almost everywhere. 


\subsection{Continuous functions in the model}

We now consider the function from $\mathbb{R}$ into $\mathbb{R}$ in the model i.e. $u \in V^{(\mathcal{B})}$ satisfying $\llbracket u: \mathbb{R} \rightarrow \mathbb{R} \rrbracket=I$.

Proposition 1.4.2, Chapter 1 implies that there is a $1-1$ correspondence between $u$ 's satisfying $\llbracket u: \mathbb{R} \rightarrow \mathbb{R} \rrbracket=I$ and the extensional functions from $\mathbb{R}^{(\mathcal{B})}$ into $\mathbb{R}^{(\mathcal{B})}$. We denote the set of all measurable functions by $\mathfrak{F}$. Then we can identify $\mathbb{R}^{(\mathcal{B})}$ with $\mathfrak{F}$. Therefore we can identify the functions from $\mathbb{R}$ into $\mathbb{R}$ in the model with the functions $F$ with the following properties.

1) $F: \mathfrak{F} \rightarrow \mathfrak{F}$.

2) extensionality for $F$, that is,

$$
\forall f, g \in \mathfrak{F} \quad\{x \mid f(x)=g(x)\} / \mathcal{T} \subseteq\{x \mid F(f)(x)=F(g) x\} / \mathcal{T} .
$$

Now we consider $\mathbb{R}$ as a measure space by providing it with the Lebesgue measure. We also think of $\mathbb{R} \times X$ as a measure space with the product measure.

Definition 2.3.1. A function $G: \mathbb{R} \times X \rightarrow \mathbb{R}$ is strongly measurable with respect to the variable $x \in X$ iff for every $\alpha \in \mathfrak{F}, G(\alpha(x), x)$ is a measure function of $x$.

Let $G: \mathbb{R} \times X \rightarrow \mathbb{R}$ be strongly measurable with respect to $x$. Define $F: \mathfrak{F} \rightarrow$ $\mathfrak{F}$ by

$$
F(\alpha)(x)=G(\alpha(x), x) .
$$

Then $F$ satisfies the extensionality condition and represents a function from $\mathbb{R}$ into $\mathbb{R}$ in the model.

Remark. If $G(a, x)$ is strongly measurable with respect to $x$, then $G(a, x)$ is measurable with respect to $(a, x)$. But the converse is not true.

Strong measurability is very abstract. We define a very useful subclass of the strong measurable functions.

Definition 2.3.2. A function $G: \mathbb{R} \times X \rightarrow \mathbb{R}$ is a B-function iff $G$ is a member of a smallest family $\mathcal{G}$ satisfying the following conditions.

1. If $f: \mathbb{R} \rightarrow \mathbb{R}$ and $g: X \rightarrow \mathbb{R}$ are measurable, then

$$
f(a) \cdot g(x) \in \mathcal{G}
$$

2. If $G_{1}(a, x)$ and $G_{2}(a, x) \in \mathcal{G}$, then

$$
G_{1}(a, x)+G_{2}(a, x) \in \mathcal{G}, \quad \text { and } \quad G_{1}(a, x) \cdot G_{2}(a, x) \in \mathcal{G} .
$$

3. If $G_{i}(a, x)(i=0,1,2, \ldots) \in \mathcal{G}$, then

$$
\underset{i \rightarrow \infty}{L} G_{i}(a, x) \in \mathcal{G} \text {, where } \underset{i \rightarrow \infty}{L} a_{i} \text { is defined by }
$$




$$
\underset{i \rightarrow \infty}{L} a_{i}= \begin{cases}\lim _{i \rightarrow \infty} a_{i} & \text { if the limit exists } \\ 0 & \text { otherwise. }\end{cases}
$$

Proposition 2.3.1. If $G$ is a B-function, then $G$ is strongly measurable.

Proof. The proposition is obvious since $\underset{i \rightarrow \infty}{L} f_{i}(x)$ is measurable if all $f_{i}(x)(i=$ $0,1,2, \ldots)$ are measurable.

Definition 2.3.3. A strongly measurable function $G(a, x)$ is pseudo-continuous iff for every sequence $\alpha_{0}, \alpha_{1}, \alpha_{2}, \ldots$ of measurable functions with

$$
\lim _{i \rightarrow \infty} G\left(\alpha_{i}(x), x\right)=G(\alpha(x), x) \quad \text { almost everywhere }
$$

provided that

$$
\lim _{i \rightarrow \infty} \alpha_{i}(x)=\alpha(x) \text { almost everywhere. }
$$

Obviously if $G$ is pseudo-continuous, $F$ defined by $F(\alpha)(x)=G(\alpha(x), x)$ represents a continuous function in the model. In this sense $G$ corresponds to a continuous function in the model. The converse is also true, that is, for every continuous function $u$ in the model, there exists a pseudo-continuous $G$ such that $G$ corresponds to $u$. We are going to prove this.

Let the following conditions hold in $V^{(\mathcal{B})}$.

1) $u: \mathbb{R} \rightarrow \mathbb{R}$ and

$$
u_{i}: \mathbb{R} \rightarrow \mathbb{R} \text { for every } i \in \omega \text {. }
$$

2) $\mathbb{R}=\bigcup_{i \in \omega} A_{i}$ and $A_{i} \cap A_{j}=\phi$ if $i \neq j$.

3) For every $a \in \mathbb{R}$

$$
u(a)=u_{i}(a) \text { if } a \in A_{i}
$$

Let $\alpha \in \mathbb{R}^{(\mathcal{B})}$ and $\llbracket \alpha \in A_{i} \rrbracket=b_{i}$. There exist $S_{0}, S_{1}, \ldots \in \mathcal{S}$ such that

1) $b_{i}=S_{i} / \mathcal{T}$ for every $i \in \omega$

2) $X=\bigcup_{i \in \omega} S_{i}$

3) $S_{i} \cap S_{j}=\phi$ if $i \neq j$.

Let $F_{i}: \mathfrak{F} \rightarrow \mathfrak{F}$ correspond to $u_{i}$ for $i \in \omega$. Define $F: \mathfrak{F} \rightarrow \mathfrak{F}$ by the following equation.

$$
F(\alpha)(x)=F_{i}(\alpha)(x) \text { if } x \in S_{i}
$$


Then obviously $F$ corresponds to $u$. We use this construction in the following. Let $f: \mathbb{R} \rightarrow \mathbb{R}$ be a continuous function let $m \in \mathbb{Z}$ and let $n$ be a positive natural number. Let $f_{n}$ be the function of the linear graph through the points $\left(m+\frac{i}{n}, f\left(m+\frac{i}{n}\right)\right)$. Then $f_{n}(x)$ goes to $f(x)$ if $n$ goes to $\infty$. Now let $\llbracket u: \mathbb{R} \rightarrow \mathbb{R}$ is continuous $\rrbracket=I$ and $F: \mathfrak{F} \rightarrow \mathfrak{F}$ represent $u$. Define $F_{n}: \mathfrak{F} \rightarrow \mathfrak{F}$ by the following equation

$$
\begin{aligned}
F_{n}(\alpha)(x)= & ((i+1)-n(\alpha(x)-m)) F\left(m+\frac{i}{n}\right)(x) \\
& +(n(\alpha(n)-m)-i) F\left(m+\frac{i+1}{n}\right)(x) \\
& \text { if } m+\frac{i}{n} \leq \alpha(x)<m+\frac{i+1}{n}
\end{aligned}
$$

where $i=0, \ldots, n-1$. Let $u_{n} \in \mathbb{R}^{(\mathcal{B})}$ be represented by $F_{n}$. We have

$$
\llbracket \forall x \in \mathbb{R} \lim _{n \rightarrow \infty} u_{n}(x)=u(x) \rrbracket=I .
$$

Define $G_{n}: \mathbb{R} \times X \rightarrow \mathbb{R}$ by the following equation.

$$
\begin{aligned}
G_{n}(a, x)= & ((i+1)-n(a-m)) F\left(m+\frac{i}{n}\right)(x) \\
& +(n(a-m)-i) F\left(m+\frac{i+1}{n}\right)(x) \\
& \text { if } m+\frac{i}{n} \leq a<m+\frac{i+1}{n}
\end{aligned}
$$

where $i=0, \ldots, n-1$. Obviously $G_{n}$ is a strongly measurable function,

$$
F_{n}(\alpha)(x)=G_{n}(\alpha(x), x),
$$

and $G_{n}$ correspond to $u_{n}$. Define $G: \mathbb{R} \times X \rightarrow \mathbb{R}$ by the following

$$
G(a, x)= \begin{cases}\lim _{n \rightarrow \infty} G_{n}(a, x) & \text { if there exists a limit } \\ 0 & \text { otherwise. }\end{cases}
$$

Then $G$ is a strongly measurable function. We claim that $G$ corresponds to $u$. Now let $\alpha \in \mathbb{R}^{(\mathcal{B})}$. Then

$$
\llbracket \lim _{n \rightarrow \infty} u_{n}(\alpha)=u(\alpha) \rrbracket=I
$$

so

$$
\left\{x \mid \lim _{n \rightarrow \infty} G_{n}(\alpha(x), x)=F(\alpha)(x)\right\} / \mathcal{T}=I
$$

i.e.

$$
\lim _{n \rightarrow \infty} G_{n}(\alpha(x), x)=F(\alpha)(x) \text { almost everywhere. }
$$


Therefore

$$
G(\alpha(x), x)=F(\alpha)(x) \text { almost everywhere }
$$

i.e. $G$ corresponds to $u$.

This also implies that $G_{n}(\alpha(x), x)$ converges to $G(\alpha(x), x)$ almost everywhere. As a special case, for every $a, G_{n}(a, x)$ converges to $G(a, x)$ almost everywhere with respect to $x$.

Now we show that $G$ is a $B$-function. For this it suffices to show that $G_{n}$ is a $B$-function for every $n$. Let $g_{m n i}(a)$ be the characteristic function of $\left\{a \mid m+\frac{i}{n} \leq a<m+\frac{i+1}{n}\right\}$. Then

$$
\begin{aligned}
G_{n}(a, x)= & \sum_{m, i}\left(g_{m n i}(a)((i+1)-n(a-m)) F\left(m+\frac{i}{n}\right)(x)\right. \\
& \left.+g_{m n i}(a)(n(a-m)-i) F\left(m+\frac{i+1}{n}\right)(x)\right)
\end{aligned}
$$

Denote the right hand side by $\sum_{m} H_{m}(a, x)$. Then

$$
\sum_{m} H_{m}(a, x)=\sum_{k \rightarrow \infty}^{L} \sum_{m=-k}^{k} H_{m}(a, x)
$$

Since $\sum_{m=-k}^{k} H_{m}(a, x)$ is a $B$-function, $G_{n}(a, x)$ is also a $B$-function. Thus we have obtained the following theorem.

Theorem 2.3.2. For every continuous function $u$ in the model, there exists a pseudo-continuous B-function $G$ which corresponds to $u$.

\subsection{Baire functions and Borel sets in the model}

Let $G_{n}(a, x)(n=0,1,2, \ldots)$ be strongly measurable functions and correspond to $u_{n}(n=0,1,2, \ldots)$ respectively where $u_{n}$ are functions from $\mathbb{R}$ into $\mathbb{R}$ in the model. Let

$$
\llbracket \forall a \in \mathbb{R} \lim _{n \rightarrow \infty} u_{n}(a)=u(a) \rrbracket=I \text {, and } G(a, x)=\underset{n \rightarrow \infty}{L} G_{n}(a, x) .
$$

Proposition 2.4.1. In this situation, $G$ corresponds to $u$.

Proof. Let $\alpha \in \mathfrak{F}$ and $F: \mathfrak{F} \rightarrow \mathfrak{F}$ represent $u$. Then

$$
G_{n}(\alpha(x), x) \text { converges to } F(\alpha)(x) \text { almost everywhere. }
$$

Therefore 


$$
G(\alpha(x), x)=F(\alpha)(x) \text { almost everywhere. }
$$

We are going to use the theory of Baire functions. Therefore we recall the definition of Baire functions.

Definition 2.4.1. Let $X$ be a topological space. A function from $X$ into $\mathbb{R}$ is called a Baire function if it belongs to the smallest family satisfying the following conditions.

1. It has all continuous functions from $X$ into $\mathbb{R}$.

2. If $f_{1}, f_{2}, \ldots$ belong to it and $\lim _{n \rightarrow \infty} f_{n}(x)=f(x)$ for every $x \in X$, then $f$ belongs to it.

It is well-known that if $X$ is a perfect set in an Euclidean space, then the family of all Baire functions coincides with the family of all Borel measurable functions.

In the last section, $\mathbb{R}$ is considered to be a measure space together with the family of Lebesgue measurable sets and the Lebesgue measure. However all the arguments there go through if $\mathbb{R}$ is made as a measure space with the family of Borel sets and the Lebesgue measure on Borel sets. For this measure space, we rename a $B$-function as a pseudo-Baire function.

Definition 2.4.2. A function $G: \mathbb{R} \times X \rightarrow \mathbb{R}$ is a pseudo-Baire function iff $G$ is a member of a smallest family $\mathcal{G}$ satisfying the following conditions.

1. If $f: \mathbb{R} \rightarrow \mathbb{R}$ is a Baire function and $g: X \rightarrow \mathbb{R}$ is measurable, then $f(a) \cdot g(x) \in \mathcal{G}$.

2. If $G_{1}, G_{2} \in \mathcal{G}$, then $G_{1}+G_{2}, G_{1} G_{2} \in \mathcal{G}$.

3. If $G_{i}(i=0,1,2, \ldots) \in \mathcal{G}$, then $\underset{i \rightarrow \infty}{L} G_{i} \in \mathcal{G}$.

Let $u$ be a continuous function in the model. Then there exists a pseudocontinuous pseudo-Baire function $G$ which corresponds to $u$.

Definition 2.4.3. A subset $B$ of $\mathbb{R} \times X$ is a pseudo-Borel set iff $B$ is a member of the smallest family $\mathcal{L}$ satisfying the following conditions.

1. If $A$ is a Borel set of $\mathbb{R}$ and $S \in \mathcal{S}$, then $A \times S \in \mathcal{L}$.

2. The family $\mathcal{L}$ is closed under Boolean operations.

3. If $B_{i}(i=0,1,2, \ldots) \in \mathcal{L}$, then $\bigcup_{i} B_{i} \in \mathcal{L}$. 
Let $\mathcal{G}$ be the family of all pseudo-Baire functions and let $\mathcal{L}$ be the family of all pseudo-Borel sets. Then it is easily seen that $\mathcal{G}$ is the family of all $\mathcal{L}$-measurable functions.

Let $B$ be a pseudo-Borel set. Define $v \in V^{(\mathcal{B})}$ with $\mathcal{D}(v)=\mathbb{R}^{(\mathcal{B})}$ as follows. For every $u \in \mathbb{R}^{(\mathcal{B})}$, take $\alpha \in \mathfrak{F}$ which corresponds to $u$. Then

$$
v(u)=\{x \mid\langle\alpha(x), x\rangle \in B\} / \mathcal{T} .
$$

Obviously $v$ is a subset of $\mathbb{R}$ in the model and we say that $B$ corresponds to $v$.

Theorem 2.4.2. The family $\mathcal{G}$ corresponds to the set of all Baire functions in the model and $\mathcal{L}$ corresponds to the set of all Borel sets in the model.

Proof. Define $W$ by the following equation.

$$
W=\{u \mid \exists g \in \mathcal{G} \text { ( } g \text { corresponds to } u)\} .
$$

Now define $w=W \times\{I\}$ i.e. $w$ is a constant function defined on $W$ whose value is $I$. We claim that

$$
\llbracket u \in w \rrbracket=I \Rightarrow \exists g \in \mathcal{G} \text { ( } g \text { corresponds to } u \text { ). }
$$

First $\llbracket u \in w \rrbracket=I$ implies the existence of a $\left\{u_{i} \mid i \in J\right\}$ and $\left\{b_{i} \mid i \in J\right\}$ such that $\left\{b_{i}\right\}_{i}$ is a partition of unity, $\left\{u_{i}\right\}_{i}$ is a subset of $W$, and

$$
u=\sum u_{i} \cdot b_{i} .
$$

Since $\mathcal{B}$ satisfies the c.c.c., the cardinality of $J$ is countable. Let $G_{i} \in \mathcal{G}(i \in J)$ correspond to $u_{i}$ and $\left\{S_{i}\right\}_{i} \subseteq S$ satisfy the following conditions.

1) $S_{i} / \mathcal{T}=b_{i}$

2) $\bigcup_{i} S_{i}=X$

3) $S_{i} \cap S_{j}=\phi$ for $i \neq j$.

Define $G: \mathbb{R} \times X \rightarrow \mathbb{R}$ by the following condition

$$
G(a, x)=G_{i}(a, x) \text { if } x \in S_{i} .
$$

Then $G \in \mathcal{G}$ and $G$ corresponds to $u$. Next we claim that for every Baire function $u$ in the model, there exists a $G \in \mathcal{G}$ which corresponds to $u$. Since there exists such a $G \in \mathcal{G}$ for the continuous function $u$ in the model, it suffices to show the existence of such a $G \in \mathcal{G}$ for $u$ with $\llbracket u=\lim _{i \rightarrow \infty} u_{i} \rrbracket=I$ assuming the existence of $G_{i} \in \mathcal{G}$ for each $u_{i}$. Now the existence of $G$ is obvious since we can take $G=\underset{i \rightarrow \infty}{L} G_{i}$.

Now, define $W_{0}$ and $w_{0}$ by

$$
W_{0}=\{u \mid \exists B \in \mathcal{L}(B \text { corresponds to } u)\},
$$


and $w_{0}=W_{0} \times\{I\}$. Then in the same way for every Borel set $u$ in the model, there exists a $B \in \mathcal{L}$ such that $B$ corresponds to $u$.

The converse is also true, that is, every $B \in \mathcal{L}$ corresponds to some Borel set $u$ in the model. If $B$ is $A \times X$, then $B$ corresponds to $\check{A}$. If $B$ is $\mathbb{R} \times S$, then $B$ corresponds to $\mathbb{R}^{(\mathcal{B})} \cdot b+\check{\phi} \cdot(I-b)$ where $b=S / \mathcal{T}$. In this correspondence, Boolean operations and $\bigcup_{i \in \omega}$ are preserved. Therefore our claim is proved. In the same way, it is easily seen that every $G \in \mathcal{G}$ corresponds to some Baire function in the model.

\subsection{Integration and differentiation in the model}

Let $\llbracket u: \mathbb{R} \rightarrow \mathbb{R}$ is differential and its derivative is $v \rrbracket=I$ and let $G$ and $H$ corresponds to $u$ and $v$ respectively.

Let $\alpha, \beta \in \mathfrak{F}$ and $\beta(x) \neq 0$ hold almost everywhere. Then

$$
\lim _{\beta(x) \rightarrow 0} \frac{G(\alpha(x)+\beta(x), x)-G(\alpha(x), x)}{\beta(x)}=H(\alpha(x), x)
$$

almost everywhere.

Therefore

$$
\frac{\partial G}{\partial a}(a, x)=H(a, x) \text { almost everywhere. }
$$

On the other hand, let $G$ and $H$ be pseudo-Baire functions and for every $a$,

$$
\frac{\partial G}{\partial a}(a, x)=H(a, x) \text { almost everywhere. }
$$

Then for every $\alpha, \beta_{0}, \beta_{1}, \beta_{2}, \ldots \in \mathfrak{F}$ such that $\beta_{i}(x) \neq 0$ almost everywhere $(i=0,1,2, \ldots)$ and $\lim _{i \rightarrow \infty} \beta_{i}(x)=0$ almost everywhere

$$
\lim _{i \rightarrow \infty} \frac{G\left(\alpha(x)+\beta_{i}(x), x\right)-G(\alpha(x), x)}{\beta_{i}(x)}=\frac{\partial}{\partial \alpha} G(\alpha(x), x)
$$

almost everywhere.

Therefore we have the following theorem.

Theorem 2.5.1. Let $G(a, x)$ be a pseudo-Baire function, such that $G$ corresponds to $u$, and $\frac{\partial}{\partial a} G(a, x)$ exists almost everywhere for all $a$. Then

$$
\llbracket u \text { is differentiable } \rrbracket=I
$$

and, if $\frac{\partial}{\partial a} G(a, x)$ corresponds to $v$, then

$$
\llbracket \text { the derivative of } u \text { is } v \rrbracket=I \text {. }
$$


On the other hand, if $\llbracket$ the derivative of $u$ is $v \rrbracket=I$ and $u$ and $v$ correspond to $G$ and $H$ respectively, then

$$
\frac{\partial}{\partial a} G(a, x)=H(a, x) \text { almost everywhere for all } a \text {. }
$$

Let $\llbracket u: \mathbb{R} \rightarrow \mathbb{R}$ is a Baire function $\rrbracket=I$ and $G: \mathbb{R} \times X \rightarrow \mathbb{R}$ be a pseudo-Baire function corresponding to $u$. The relation between $\int_{\mathbb{R}} u(t) d t$ and $\int_{\mathbb{R}} G(a, x) d a$ is discussed in the following theorem.

Theorem 2.5.2. $\llbracket u$ is integrable $\rrbracket=I$ is equivalent to ' $G(a, x)$ is integrable for almost all $x$ '. If $\llbracket u$ is integrable $=I$, then $\int_{\mathbb{R}} G(a, x) d a$ corresponds to $\int_{\mathbb{R}} u(t) d t$

Proof. Obviously $\max (0, G(a, x))$ corresponds to $\max (0, u)$. Therefore we may assume that $0 \leq G(a, x)$ and $\llbracket \forall t \in \mathbb{R} \check{0} \leq u(t) \rrbracket=I$. Also $\llbracket \int_{\mathbb{R}} u(t) d t=$ $\sum_{n \in \mathbb{Z}} \int_{n}^{n+1} u(t) d t \rrbracket=I$ and $\int_{\mathbb{R}} G(a, x) d a=\sum_{n \in \mathbb{Z}} \int_{n}^{n+1} G(a, x) d a$. Therefore it suffices to show that $\llbracket u$ is integrable on $\left[\check{n},(n+1)^{\sqrt{ }} \rrbracket \rrbracket=I\right.$ is equivalent to ' $G(a, x)$ is integrable on $[n, n+1]$ for almost all $x$ ' and $\int_{n}^{n+1} G(a, x) d a$ corresponds to $\int_{n}^{n+1} u(t) d t$ provided that $\llbracket u$ is integrable on $[\check{n},(n+1) \sqrt{ }] \rrbracket=$ I. Since $\min (M, G(a, x))$ corresponds to $\min (M, u(t)), \int_{n}^{n+1} G(a, x) d a=$ $\lim _{M \rightarrow \infty} \int_{n}^{n+1} \min (M, G(a, x)) d a$ and $\llbracket \int_{n}^{n+1} u(t) d t=\lim _{M \rightarrow \infty} \int_{n}^{n+1} \min (M, u(t)) d t \rrbracket=$ $I$, we can assume that $G(a, x) \leq M$ and $\llbracket \forall t \in \mathbb{R} u(t) \leq \check{M} \rrbracket=I$. Now let both $G_{1}(a, x)$ and $G_{2}(a, x)$ correspond to $u$. Then by Fubini's theorem,

$$
\int_{X} \int_{n}^{n+1}\left|G_{1}(a, x)-G_{2}(a, x)\right| d a d x=\int_{n}^{n+1} \int_{X}\left|G_{1}(a, x)-G_{2}(a, x)\right| d x d a=0 .
$$

Therefore

$$
\int_{n}^{n+1} G_{1}(a, x) d a=\int_{n}^{n+1} G_{2}(a, x) d a \text { almost everywhere. }
$$

Now we have only to prove the theorem for some specific $G(a, x)$ corresponding to $u$. Since $\lim _{i \rightarrow \infty} G_{i}(a, x)=G(a, x)$ implies $\lim _{i \rightarrow \infty} \min \left(M, G_{i}(a, x)\right)=\min (M, G(a, x))$ and $\int_{n}^{n+1} \lim _{i \rightarrow \infty} f_{i}(t) d t=\lim _{i \rightarrow \infty} \int_{n}^{n+1} f_{i}(t) d t$ if $0 \leq f_{i}(t) \leq M(i=0,1,2, \ldots)$, it 
suffices to prove the theorem for the continuous function $u$ in the model. We define $u_{m}$ and $G_{m}$ by the equations

$$
\begin{aligned}
u_{m}(t)= & ((i+1)-m t) u\left(n+\frac{i}{m}\right)+(m t-i) u\left(n+\frac{i+1}{m}\right) \\
& \text { if } n+\frac{i}{m} \leq t \leq n+\frac{i+1}{m} \\
& \text { for } i=0,1, \ldots, m-1
\end{aligned}
$$

and

$$
\begin{aligned}
G_{m}(a, x)= & ((i+1)-m a) F\left(n+\frac{i}{m}\right)(x)+(m a-i) F\left(n+\frac{i+1}{m}\right)(x) \\
& \text { if } n+\frac{i}{m} \leq a \leq n+\frac{i+1}{m} \\
& \text { for } i=0,1, \ldots, m-1
\end{aligned}
$$

where $F: \mathfrak{F} \rightarrow \mathfrak{F}$ represents $u$. Then $\int_{n}^{n+1} G_{m}(a, x) d a$ corresponds to $\int_{n}^{n+1} u_{m}(t) d t$. If $G(a, x)=\underset{m \rightarrow \infty}{L} G_{m}(a, x)$, then $\lim _{m \rightarrow \infty} \int_{n}^{n+1} G_{m}(a, x) d a=\int_{n}^{n+1} G(a, x) d a$ and $\llbracket \int_{n}^{n+1} u(t) d t=\lim _{m \rightarrow \infty} \int_{n}^{n+1} u_{m}(t) d t \rrbracket=I$, and hence $\int_{n}^{n+1} G(a, x) d a$ corresponds to $\int_{n}^{n+1} u(t) d t$.

Corollary 2.5.3. Let $v$ be a Borel set in the model, let $B$ be a pseudo-Borel set corresponding to $v$, and let $m$ be the Lebesgue measure in the model. Then $\int_{\mathbb{R}} \chi_{B}(a, x) d a$ corresponds to $m(v)$, where $\chi_{B}$ is the characteristic function of

Proof. We also denote the characteristic function of $v$ in the model by $\chi_{v}$. Then for every $u \in \mathbb{R}^{(B)}$ and for every $\alpha \in \mathfrak{F}$ corresponding to $u$,

$$
\begin{aligned}
\llbracket \chi_{v}(u)=\check{1} \rrbracket & =\llbracket u \in v \rrbracket \\
& =\{x \mid\langle\alpha(x), x\rangle \in B\} / \mathcal{T} \\
& =\left\{x \mid \chi_{B}(\alpha(x), x)=1\right\} / \mathcal{T} .
\end{aligned}
$$

Therefore $\chi_{B}$ corresponds to $\chi_{v}$. Since $\llbracket m(v)=\int_{\mathbb{R}} \chi_{v}(t) d t \rrbracket=I, \int_{\mathbb{R}} \chi_{B}(a, x) d a$ corresponds to $m(v)$.

Corollary 2.5.4. Let $\llbracket u: \mathbb{R} \rightarrow \mathbb{R}$ is a Baire function $\rrbracket=I, \llbracket v \subseteq \mathbb{R}$ is a Borel set $\rrbracket=I, G: \mathbb{R} \times X \rightarrow \mathbb{R}$ be a pseudo-Baire function corresponding to u, and $B \subseteq \mathbb{R} \times X$ be a pseudo-Borel set corresponding to $v$. For every $x \in X$, we denote $\{a \in \mathbb{R} \mid\langle a, x\rangle \in B\}$ by $B_{x}$. Then $\int_{B_{x}} G(a, x) d a$ corresponds to $\int_{v} u(t) d t$. 
Proof. Since $\llbracket \int_{v} u(t) d t=\int_{\mathbb{R}} \chi_{v}(t) \cdot u(t) d t \rrbracket=I$ and $\chi_{B} \cdot G(a, x)$ corresponds to $\chi_{v} \cdot u, \int_{\mathbb{R}} \chi_{B}(a, x) G(a, x) d a$ corresponds to $\int_{v} u(t) d t$. The corollary follows immediately from this.

We are going give an interpretation of Baire category theorem. In order to understand the meaning of this interpretation, the following definition is useful.

Definition 2.5.1. A measure space $(X, \mathcal{S}, \mu)$ is a Borel measure space iff $X$ is a topological space and $\mathcal{S}$ is the family of all Borel sets in $X$.

If $(X, \mathcal{S}, \mu)$ is a Borel measure space and $X$ is perfectly separable, then a pseudo-Borel set in $\mathbb{R} \times X$ is nothing but a Borel set in $\mathbb{R} \times X$ and a pseudo-Baire function: $\mathbb{R} \times X \rightarrow \mathbb{R}$ is nothing but a Borel-measurable function: $\mathbb{R} \times X \rightarrow \mathbb{R}$.

Definition 2.5.2. Let $B$ be a pseudo-Borel set of $\mathbb{R} \times X$ and let $f \in \mathfrak{F}$. Then $f<B$ is defined to be ' $\langle f(x), x\rangle \in B$ almost everywhere.' The set $B$ is pseudodense in $\mathbb{R} \times X$ iff $\forall \varepsilon>0 \forall f \in \mathfrak{F} \exists g \in \mathfrak{F}(g<B$ and $|f(x)-g(x)|<\varepsilon$ almost everywhere). Obviously if $B$ corresponds to $u$ in the model, $B$ is pseudo-dense in $\mathbb{R} \times X$ iff $u$ is dense in $\mathbb{R}$ in the model.

Definition 2.5.3. Let $B$ be a pseudo-Borel set in $\mathbb{R} \times X$ and let $f \in \mathfrak{F}$ be such that $f<B$. Let $D \in \mathcal{S}$ and $\varepsilon>0$. Then $D$ is a domain of $U(f, \varepsilon)$ in $B$ iff

$\forall g \in \mathfrak{F}(x \in D \wedge|f(x)-g(x)|<\varepsilon \Rightarrow\langle g(x), x\rangle \in B$ almost everywhere).

Proposition 2.5.5. Let $B$ be a pseudo-Borel set in $\mathbb{R} \times X$ corresponding to $v$ in the model, let $f \in \mathfrak{F}$ correspond to $u$ in the model and $f<B$. Let $D \in \mathcal{S}$, let $\varepsilon>0$ be a rational and let $b=D / \mathcal{T}$. Then $D$ is a domain of $U(f, \varepsilon)$ in $B$ iff $\llbracket U(u, \check{\varepsilon}) \subseteq v \rrbracket \geq b$, where $U(u, \check{\varepsilon})$ is the $\check{\varepsilon}$-neighborhood of $u$ in the model.

Proof.

$$
\begin{aligned}
\llbracket U(u, \check{\varepsilon}) \subseteq v \rrbracket & =\llbracket \forall x \in \mathbb{R}|x-u|<\check{\varepsilon} \Rightarrow x \in v \rrbracket \\
& =\inf _{u_{1} \in \mathbb{R}^{(B)}} \llbracket\left|u_{1}-u\right|<\check{\varepsilon} \Rightarrow u_{1} \in v \rrbracket \\
& =\{x \mid \forall g \in \mathfrak{F}(|f(x)-g(x)|<\varepsilon \Rightarrow g<B)\} / \mathcal{T}
\end{aligned}
$$

Therefore $\llbracket U(u, \varepsilon) \subseteq v \rrbracket \geq b$ iff

$$
\{x \mid \forall g \in \mathfrak{F}(|f(x)-g(x)|<\varepsilon \Rightarrow\langle g(x), x\rangle \in B)\} / \mathcal{T} \geq D / \mathcal{T},
$$

whence follows the proposition.

Definition 2.5.4. A pseudo-Borel set $B$ in $\mathbb{R} \times X$ is pseudo-open iff for every $f \in \mathfrak{F}$ with $f<B$, there exists a $D_{n} \in \mathcal{S}$, a domain of $U\left(f, \frac{1}{2^{n}}\right)$ in $B(n=$ $0,1,2, \ldots)$ such that

$$
X=\bigcup_{n} D_{n} \text { almost everywhere }
$$


that is

$$
\mu\left(X-\bigcup_{n} D_{n}\right)=0 .
$$

Proposition 2.5.6. Let $B$ be a pseudo-Borel set in $\mathbb{R} \times X$ corresponding to $v$ in the model. Then $B$ is pseudo-open iff $\llbracket v$ is open in $\mathbb{R} \rrbracket=I$.

Proof. Suppose that $B$ is pseudo-open. Let $f \in \mathfrak{F}$ correspond to $u$ and $f<B$. Take $D_{n}$ as in the definition of 'pseudo-open'. If $b_{n}=D_{n} / \mathcal{T}$, then $\sup b_{n}=I$ and $\llbracket U\left(u, \frac{1}{2^{n}}\right) \subseteq v \rrbracket \geq b_{n}$. If $r=\sum_{n} \frac{1}{2^{n}}\left(b_{n}-\left(b_{n-1}+\cdots+b_{0}\right)\right)$, then $r$ is a rational in the model, $\llbracket r>\check{0} \rrbracket=I$ and

$$
\llbracket U(u, r) \subseteq v \rrbracket=I
$$

Therefore $\llbracket v$ is open $\rrbracket=I$. Now suppose that $\llbracket v$ is open $\rrbracket=I$. Let $f \in \mathfrak{F}$ correspond to $u$ and $f<B$. Then

$$
\llbracket \exists r \in \mathbb{Q}(r>0 \wedge U(u, r) \subseteq v) \rrbracket=I .
$$

Therefore there exists an $r=\sum_{i} r_{i} \cdot b_{i}$ such that

$$
\llbracket U(u, r) \subseteq v \rrbracket=I
$$

where the $r_{i}$ are positive rationals and $\left\{b_{i}\right\}_{i}$ is a partition of unity. Let $b_{i}=S_{i} / \mathcal{T}$ and $D_{n}=\bigcup\left\{S_{i} \mid r_{i} \geq \frac{1}{2^{n}}\right\}$. The proposition now follows from Proposition 2.5.5.

Now we get the following interpretation of the Baire category theorem.

Theorem 2.5.7. If $B_{n}(n=0,1,2, \ldots)$ is pseudo-open and pseudo-dense in $\mathbb{R} \times X$, then $\bigcap_{n} B_{n}$ is also pseudo-dense.

\subsection{Relation between projection algebras and measure algebras}

Let $\mathcal{H}$ be a Hilbert space and $\mathcal{B}$ be a complete Boolean algebra of projection in $\mathcal{H}$. If $\mathcal{H}$ is separable, then $\mathcal{B}$ is closely related with a measure algebra. The base of our discussion is the following spectral theorem. (See [7]).

Theorem 2.6.1 (spectral theorem). Let $\mathcal{H}$ be separable. If $\left\{A_{\alpha}\right\}$ is a set of commutable self-adjoint operators on $\mathcal{H}$, then there is a measure space $(X, \mu)$ and a unitary map $U: \mathcal{H} \rightarrow \mathcal{L}^{2}(X, \mu)$ such that $U A_{\alpha} U^{-1}$ is multiplication by a real measurable function on $\tilde{A}_{\alpha}$. If $\tilde{B}$ is any real measurable function on $X$ and $M_{\tilde{B}}$ is the corresponding multiplication operator then $B=U^{-1} M_{\tilde{B}} U$ is a self-adjoint operator on $\mathcal{H}$ which commutes with the $A_{\alpha}$. 
Let $\mathcal{H}$ be separable and $\mathcal{B}$ be a complete Boolean algebra of projections in $\mathcal{H}$. Extend $\mathcal{B}$ to a maximal complete Boolean algebra $\tilde{\mathcal{B}}$ of projections in $\mathcal{H}$. Let $\left\{A_{\alpha}\right\}$ in the Theorem 2.6.1 be $\tilde{\mathcal{B}}$ and $(X, \mu)$ be the measure space described in the theorem. If $P \in \mathcal{B}$, then $P^{2}=P$ and the real measurable function $\tilde{P}$ corresponding $P$ must take either 0 or 1 as its values almost everywhere. Therefore $\tilde{P}$ is represented by a characteristic function of a measurable set $S_{P}$. $A$ measurable set $S_{P}$ is uniquely determined by $P$ in the sense that $S_{P}^{\prime}$ is equal to $S_{P}$ up to a measure zero set if $S_{P}^{\prime}$ also corresponds to $P$. On the other hand, let $S$ be a measurable set and $\tilde{S}$ be the multiplication operator of the characteristic function of $S$. Then $\tilde{S}$ is a projection operator on $\mathcal{L}^{2}(X, \mu)$ and $U^{-1} \tilde{S} U$ is a projection operator on $\mathcal{H}$ commuting with all members of $\tilde{\mathcal{B}}$. Since $\tilde{\mathcal{B}}$ is maximal, $U^{-1} \tilde{S} U$ must be a member of $\tilde{\mathcal{B}}$. Let $\tilde{\mathcal{B}}_{0}$ be the measure algebra of $(X, \mu)$. Then the assignment $P \mapsto S_{P}$ is clearly an isomorphism between $\tilde{\mathcal{B}}$ and $\tilde{\mathcal{B}}_{0}$. Let $\mathcal{B}_{0}$ be the image of $\mathcal{B}$ under the isomorphism. From the definition of a complete Boolean algebra of projections follows that for each $A \subseteq|\mathcal{B}|$

$$
\sup ^{\mathcal{B}} A=\sup ^{\tilde{\mathcal{B}}} A
$$

and

$$
\inf ^{\mathcal{B}} A=\inf ^{\tilde{\mathcal{B}}} A
$$

that is, a class $A \subseteq|\mathcal{B}|$ has the same sup and inf relative to $\mathcal{B}$ that it has relative to $\tilde{\mathcal{B}}$. By the isomorphism, we have that each subset of $\left|\mathcal{B}_{0}\right|$ has the same sup and inf relative to $\mathcal{B}_{0}$ that it has relative to $\tilde{\mathcal{B}}_{0}$. Thus $\mathcal{B}_{0}$ is a complete subalgebra of $\tilde{\mathcal{B}}_{0}$. The isomorphism between $\tilde{\mathcal{B}}$ and $\tilde{\mathcal{B}}_{0}$ induces an isomorphism between $V^{(\tilde{\mathcal{B}})}$ and $V^{\left(\tilde{\mathcal{B}}_{0}\right)}$ and also an isomorphism between $V^{(\mathcal{B})}$ and $V^{\left(\mathcal{B}_{0}\right)}$. Therefore we can make a correspondence between notions on $V^{(\mathcal{B})}$ or $V^{(\tilde{\mathcal{B}})}$ in Chapter 1 and notions on $V^{\left(\mathcal{B}_{0}\right)}$ or $V^{\left(\tilde{\mathcal{B}}_{0}\right)}$ in this chapter. One minor trouble is that we defined a real number to the upper half line in a Dedekind cut in $V^{\left(\tilde{\mathcal{B}}_{0}\right)}$ and to be the lower half line without the end point in a Dedekind cut in $V^{\left(\tilde{\mathcal{B}}_{0}\right)}$. However this comes from a very minor technical convenience and now we define a real number to be the upper half line in both $V^{(\tilde{\mathcal{B}})}$ and $V^{\left(\tilde{\mathcal{B}}_{0}\right)}$. Everything in this chapter goes through in the same way even if the definition of a real number is changed in this way. Now let $A$ be a self-adjoint operator in $(\tilde{\mathcal{B}})$. Then $A$ is a real in $V^{(\tilde{\mathcal{B}})}$. Let $A_{0}$ be the image of $A$ under the isomorphism between $V^{(\tilde{\mathcal{B}})}$ and $V^{\left(\tilde{\mathcal{B}}_{0}\right)}$. Then $A_{0}$ is a real in $V^{\left(\tilde{\mathcal{B}}_{0}\right)}$. Therefore $A_{0}$ is a measurable function in $(X, \mu)$. This is exactly the correspondence between self-adjoint operators and multiplication operators of real measurable functions of $(X, \mu)$ in Theorem 2.6.1.

Now what is a real number in $V^{\left(\mathcal{B}_{0}\right)}$ ? Let $\mathcal{T}$ be the ideal of all measure zero sets in $(X, \mu)$. A real measurable function $f$ of $(X, \mu)$ is said to be $\mathcal{B}_{0^{-}}$ measurable if for every $r \in \mathbb{R},\{x \in X \mid f(x)<r\} / \mathcal{T}$ is a member of $\mathcal{B}_{0}$. Then a real number in $V^{\left(\mathcal{B}_{0}\right)}$ obviously corresponds to a real $\mathcal{B}_{0}$-measurable function of $(X, \mu)$.

Let $f: \mathbb{R} \rightarrow \mathbb{R}$ be continuous and $A$ be a self-adjoint operator in $(\tilde{\mathcal{B}})$. If $\sum r_{i} P_{i}$ is a rational in $(\tilde{\mathcal{B}})$, then $f\left(\sum r_{i} P_{i}\right)=\sum f\left(r_{i}\right) P_{i}$. If $\left\{\sum r_{i} P_{i}\right\}$ converges 
to $(A)$ in $V^{(\tilde{\mathcal{B}})}$, then $\sum f\left(r_{i}\right) P_{i}$ converges to $f(A)$ in $V^{(\tilde{\mathcal{B}})}$. Let $g$ be a real measurable function of $(X, \mu)$ corresponding to $A$. Let $h$ be a real measurable function corresponding to $\sum r_{i} P_{i}$. Then $h$ is a step function satisfying the following condition

$$
h(x)=f\left(r_{i}\right) \text { if } x \in S_{P_{i}}
$$

where $S_{P_{i}}$ is a measurable set corresponding to $P_{i}$. If $\sum f\left(r_{i}\right) P_{i}$ converges to $A$, then $\sum f\left(r_{i}\right) P_{i}$ converges to $f(A)$. Since $h(x)$ converges to $f(g(x))$ almost everywhere, $f(g(x))$ corresponds to $f(A)$.

Let $f^{\prime}(x)$ be also continuous. In the terminology of $\S 2.3, f(a)$ is strongly measurable. Then Theorem 2.5.1 shows that the derivative of $f$ at $g(x)$ in $V^{\left(\tilde{\mathcal{B}}_{0}\right)}$ is $f^{\prime}(g(x))$. If $g$ is $\mathcal{B}_{0}$-measurable, then the derivative of $f$ at $g(x)$ in $V^{\left(\mathcal{B}_{0}\right)}$ is also $f^{\prime}(g(x))$. This is equivalent to the theorem that the derivative of $f$ at $A$ in $V^{(\mathcal{B})}$ is $f^{\prime}(A)$.

Now let $A_{0}, A_{1}, A_{2}, \ldots, A$ be self-adjoint operators in $(\mathcal{B})$ and $\left\|A_{0}\right\|,\left\|A_{1}\right\|,\left\|A_{2}\right\|, \ldots$ have a uniform bound, let $f_{0}(x), f_{1}(x), f_{2}(x), \ldots, f(x)$ be real $\mathcal{B}_{0}$-measurable function corresponding to $A_{0}, A_{1}, A_{2}, \ldots$ respectively. Then $\left|f_{i}(x)\right| \leq M$ almost everywhere. Suppose that $A_{0}, A_{1}, A_{2}, \ldots$ converges to $A$ in $V^{(\overline{\mathcal{B}})}$. Then $f_{0}(x), f_{1}(x), f_{2}(x), \ldots$ converges to $f(x)$ almost everywhere. Now let $g \in \mathcal{L}^{2}(X, \mu)$. Then $f_{0}(x) g(x), f_{1}(x) g(x), f_{2}(x) g(x), \ldots$ converges to $f(x) g(x)$ in $\mathcal{L}^{2}(X, \mu)$ by Lebesgue's convergence theorem. Thus $A_{0}, A_{1}, A_{2}, \ldots$ converges to $A$ in the strong topology. This is Theorem 1.6.1, Chapter 1. 


\section{Bibliography}

[1] J. Aczél: On Applications and Theory of Functional Equations, Birkhauser Verlag, Basel, 1969.

[2] G. Birkhoff: Lattice Theory, Am. Math. Sci. Coll. Publ., 1948.

[3] G. Birkhoff and J. von Neumann: The Logic of Quantum Mechanics, Ann. of Math. 37, 823, 1936.

[4] A. Church: Conditioned Disjunction as a Primitive Connective for the Propositional Calculus, Portugaliae Math. 7, Fax. 2, pp. 87-90, 1948.

[5] J. M. Jauch: Foundation of Quantum Mechanics, Addison-Wesley, 1968.

[6] C. Picon: Foundations of Quantum Physics, W. A. Benjamin, 1976.

[7] M. Reed and B. Simon: Methods of Mathematical Physics, Vol. 1, Functional Analysis, Academic Press, 1972.

[8] H. Rasiowa and R. Sikorski: The mathematics of metamathematics, Warszawa, 1963.

[9] D. Scott: Boolean Valued Models and non-standard analysis, in Applications of model theory to algebra, analysis and probability, Holt, Reinhart and Winston, 1969.

[10] G. Takeuti and W. M. Zaring: Axiomatic Set Theory, Springer-Verlag, Heidelberg, 1973.

[11] V. S. Varadarajan: Geometry of Quantum Theory Vol. 1 \& 2, D. Van Nostrand and Co., New York, 1968 \& 1970.

[12] P. Vopěnka: The Limits of Sheaves and Applications on Constructions of Models, Bull. Acad. Polon. Sci. Sér. Sci. Math., Astron. Phys. 13, 189-192, 1965.

[13] P. Vopěnka: General Theory of $\nabla$-Models, Comment. Math. Univ. Carolinae 8, 145-170, 1967. 



\section{Part II}

\section{A Conservative Extension of Peano Arithmetic}



In my opinion there is a wide gap between logic and mathematics. Let me illustrate with two examples.

1. In recursive function theory, almost all interesting recursive functions are not primitive, but almost all recursive functions, found in mathematical practice, are primitive recursive*

2. In logic, we can easily construct many arithmetical statements that are not provable in Peano arithmetic, but we hardly find any such statement in mathematical practice*.

One explanation of the second fact might be the following. When we learned to formalize mathematics, the formalization itself was an important but difficult task. Naturally we chose a very strong system so that it was easy to see that everything could be formalized in the system. It is likely that we do not need such a strong system and hence our identification of mathematical practice with a certain strong formal system could be an illusion.

Here we will define a conservative extension of Peano arithmetic and develop analysis systematically in it. We will show that theorems which can be proved in analytic number theory can be proved in Peano arithmetic.

First of all, the system of our concern is a conservative extension, hence any arithmetical proposition provable in our system is in fact provable in Peano arithmetic. My main objective will be to show that analysis can be done using only arithmetic comprehension. Indeed, much of modern mathematics has in fact an interpretation in a conservative extension of Peano arithmetic, that is, in a very weak system. This may, in a way, serve as a characteristic of current mathematics. In other words, it suggests that in spite of its powerful look, current mathematics has progressed, in essence, along arithmetic lines.

As for the conservative extension of Peano arithmetic, we will take a simple weak system, whose proof-theoretic nature is very transparent. Since this system's proof-theoretic nature is very clear, we expect that there should be very nice functional interpretation of it.

This might be useful for our first question since functional interpretations of many theorems in analysis will produce many candidates of mathematical recursive functions that are not primitive recursive. In order to prevent false optimism, let us discuss the matter in more detail. There is a good candidate of a mathematical recursive function that is not primitive recursive i.e. a theorem of van der Waerden asserts that the following function $f(l)$ is recursive.

$f(l)=\mu x$ (every division of $\{1, \ldots, x\}$ into 2 classes contains 1 class with an arithmetical progression of length $l$ ).

The known proofs of the theorem provide only non-primitive recursive bound for $f(l)$. However whether $f(l)$ is primitive recursive or not is a difficult open problem. This shows us that the difficulty of the problem is to establish that a

${ }^{*}$ Recent discovery of Kirby, Paris and Harrington on homogeneous partition relation is a great advance for these two questions.

Gentzen's $\varepsilon_{0}$-induction is also a beautiful exception for 2 . But it comes from metamathematical motivation. 
candidate is not primitive recursive rather than to find many candidates. Nevertheless we still would like to have more candidates and we would feel better if they come from the mathematical domains like number theory or analysis. There is another source of candidates in what is called primitive recursive analysis. In order to explain this, let $\gamma$ be Euler's constant. The number $\gamma$ is primitive recursive definable if definition of real numbers are given by a Cauchy sequence together with a modulus of convergence. However it is open whether $\gamma$ is primitive recursive definable if real numbers are given by Dedekind cuts, i.e. the function $f(p, q)$ for natural numbers $p, q$ defined by the following conditions seems a reasonable candidate of a recursive function that is not primitive recursive.

$$
\begin{array}{ll}
f(p, q)=0 & \text { if } \frac{p}{q}<\gamma \\
f(p, q)=1 & \text { otherwise. }
\end{array}
$$

In general, it is immaterial for recursive analysis if a real number is a Dedekind cut or a Cauchy sequence. However the distinction is important in primitive recursive analysis. Since our system is arithmetical, this is blurred in our formalization.

An additional difficulty with our use of a strong system for the formalization is that it might mislead us by causing us to forget a certain problem: Let $\mathcal{L}$ be the language of arithmetic consisting of $0,1,+, \cdot$, exp., and $=$ and let $\mathcal{S}$ be a subsystem of quantifierfree primitive recursive arithmetic restricted to the language $\mathcal{L}$. Since $\mathcal{S}$ is much weaker than quantifierfree primitive recursive arithmetic, the following conjecture seems to me very reasonable.

$$
a^{n}+b^{n}=c^{n} \rightarrow n=1 \vee n=2 \vee a=0 \vee b=0
$$

is not provable in $\mathcal{S}$.

Though this conjecture can be considered as a problem of proof theory, proof theory, at this moment, offers little hope of a solution.* It may well be that an implicit purpose of our work is to bring such problems to surface and focus attention on them.

Preliminary. Let $\tilde{L}$ and $L$ be two languages. Then $L \subset \tilde{L}$ iff every formula of $L$ is a formula of $\tilde{L}$. Let $T$ and $\tilde{T}$ be a theory of $L$ and $\tilde{L}$ respectively. Then $\tilde{T}$ is a conservative extension of $T$ iff for every formula $A$ of $L$,

$$
(T \vdash A) \leftrightarrow(\tilde{T} \vdash A) .
$$

\section{Examples.}

1. Let $T$ be a theory of a language $L$ and let $T \vdash \exists x A(x)$ where $\exists x A(x)$ is a closed formula. If $c$ is an individual constant not contained in $L$, then $T \cup\{A(c)\}$ is a conservative extension of $T$. This is a basic fact used in the usual proof of the completeness theorem. Similarly if $T \vdash$

\footnotetext{
${ }^{*}$ There is a beautiful model theoretic proof by Shepherdson [22] for a little weaker problem.
} 
$\forall x_{1} \cdots \forall x_{n} \exists y B\left(x_{1}, \ldots, x_{n}, y\right)$ for a closed formula $\forall x_{1} \cdots \forall x_{n} \exists y B\left(x_{1}, \ldots, x_{n}, y\right)$ and $f$ is a function constant not contained in $L$, then

$$
T \cup\left\{\forall x_{1} \ldots \forall x_{n} B\left(x_{1}, \ldots, x_{n}, f\left(x_{1}, \ldots, x_{n}\right)\right)\right\}
$$

is a conservative extension of $T$.

2. Bernays-Gödel Set theory is a conservative extension of ZF Set theory.

3. Let $T$ be a first order theory and let $T$ and $\tilde{T}$ be obtained from $T$ by adding type theory with full comprehension axioms. Then $\tilde{T}$ is a conservative extension of $T$. This is an implication of the completeness theorem or the cut elimination theorem of higher type.

4. In [14], Kreisel proved that the theory of nonstandard analysis is a conservative extension of ordinary analysis. 



\section{Chapter 3}

\section{Real Analysis}

\subsection{The first system}

We use the higher type language. The use of higher type language is very convenient since it is the natural language for analysis and we can take all the definitions in analysis as they are without any change. We talk about "types" rather than "orders".

Definition 3.1.1. Finite types: 0 is a finite type (the basic one); if $\tau_{1}, \ldots, \tau_{n}$ are finite types, then $\tau=\left[\tau_{1}, \ldots, \tau_{n}\right]$ is also; those are the only finite types. "Finite" will be omitted most of the time. Type [0] is also called type 1.

Definition 3.1.2 (Language). 1. Free and bound variables of each type. We use metavariables $a, b, c, \ldots, x, y, z, \ldots, \alpha, \beta, \gamma, \ldots$ and $\varphi, \psi, \chi, \ldots$ respectively in order to denote free variables of type 0 , bound variables of type 0 , free variables of higher types and bound variables of higher types.

2. Individual constants. 0,1 .

3. Function symbols.,$+ \cdot$.

4. Predicate constants. $=,<$.

Definition 3.1.3 (Terms, formulas and abstracts).

Terms are defined as follows.

1. 0,1 , and a free variable of type 0 is a term.

2. If $t_{1}$ and $t_{2}$ are terms, so are $t_{1}+t_{2}$ and $t_{1} \cdot t_{2}$.

3. Those are the only terms.

Terms are also called abstracts of type 0 .

1. If $t_{1}$ and $t_{2}$ are terms, then $t_{1}=t_{2}$ and $t_{1}<t_{2}$ are formulas. 
2. Suppose $\alpha_{1}, \ldots, \alpha_{n}, \alpha$ are free variables of type $\tau_{1}, \ldots, \tau_{n}, \tau$ respectively and $\tau=\left[\tau_{1}, \ldots, \tau_{n}\right]$. Then $\alpha\left[\alpha_{1}, \ldots, \alpha_{n}\right]$ is a formula.

3. If $A$ and $B$ are formulas, so are $\neg A$ and $A \wedge B$.

4. If $A(\alpha)$ is a formula, if $\alpha$ is a free variable of type $\tau$, and if $\varphi$ is a bound variable of type $\tau$ not occurring in $A(\alpha)$, then $\forall \varphi A(\varphi)$ is a formula. (This includes the case that $\tau$ is 0 i.e. the case which produces $\forall x A(x)$.)

5. A formula $A$ is said to be arithmetical if $A$ does not have any higher type quantifier.

6. If $A\left(\alpha_{1}, \ldots, \alpha_{n}\right)$ is an arithmetical formula, $\alpha_{1}, \ldots, \alpha_{n}$ are of type $\tau_{1}, \ldots, \tau_{n}$ respectively, and $\varphi_{1}, \ldots, \varphi_{n}$ are new distinct bound variable of type $\tau_{1}, \ldots, \tau_{n}$, then $\left\{\varphi_{1}, \ldots, \varphi_{n}\right\} A\left(\varphi_{1}, \ldots, \varphi_{n}\right)$ is an abstract of type $\left[\tau_{1}, \ldots, \tau_{n}\right]$.

7. If $\alpha$ is a free variable of type $\left[\tau_{1}, \ldots, \tau_{n}\right]$ and $V_{1}, \ldots, V_{n}$, are abstract of type $\tau_{1}, \ldots, \tau_{n}$ respectively, then $\alpha\left[V_{1}, \ldots, V_{n}\right]$ is a formula.

8. Those are the only formulas and abstracts.

It should be remarked that every abstract has no higher type quantifiers i.e. every abstract is arithmetical.

A formula defined either as in 1,2 , or in 7 is called atomic. Note that a formula defined as in 7 may contain a lot of logical symbols. We have not defined a formula of the form $V\left[V_{1}, \ldots, V_{n}\right]$ where $V$ is also an abstract; such a formula is defined in another way in the following sense. Let $V$ be of the form $\left\{\varphi_{1}, \ldots, \varphi_{n}\right\} A\left(\varphi_{1}, \ldots, \varphi_{n}\right)$. Then $V\left[V_{1}, \ldots, V_{n}\right]$ can be interpreted as $A\left(V_{1}, \ldots, V_{n}\right)$, where $A\left(V_{1}, \ldots, V_{n}\right)$ is obtained from $A\left(\varphi_{1}, \ldots, \varphi_{n}\right)$ by substituting $V_{1}, \ldots, V_{n}$ for $\varphi_{1}, \ldots, \varphi_{n}$ respectively. Therefore in the subsequent argument, an abstract will be used as a meta-expression except in the case of 7. The $V$ mentioned above is such an example.

The formal definition of "substitution" needs some careful procedure. Consider the substitution of $V$ for $\alpha$ in $\alpha\left[V_{1}\right]$, where $V$ is of the form $\{\varphi\} F(\varphi)$. This is done only after we define the substitution of $V_{1}$ for $\varphi$ in $F(\varphi)$. More precisely we define the height $h(\tau)$ of the type $\tau$ by $h(0)=0$ and $h\left(\left[\tau_{1}, \ldots, \tau_{n}\right]\right)=$ $\max \left(h\left(\tau_{1}\right), \ldots, h\left(\tau_{n}\right)\right)+1$, and we define the substitution of $V$ for $\alpha$ in $F(\alpha)$ by double induction on the height of type of $\alpha$ and the number of logical symbols in $F(\alpha)$. We sometimes have to change the names of bound variables in the substitution as is easily seen in the case of the substitution of $\{\chi\} \forall x G(\varphi, x)$ for $\alpha$ in $\forall x F(\alpha, x)$, since no figure of the form $\forall x(\cdots \forall x(\cdots) \cdots)$ is a formula in our definition. See pp. 171-173 in Proof Theory [23], referred to as PT from now on, for the precise treatment of the substitution, and for the notion of proof which we are going to discuss.

Since the cut elimination theorem is very useful for our purpose, we use Gentzen's sequent. A sequent is of the form

$$
A_{1}, \ldots, A_{m} \rightarrow B_{1}, \ldots, B_{n}
$$


where $A_{1}, \ldots, A_{m}, B_{1}, \ldots, B_{n}$ are formulas and $m, n=0,1,2, \ldots A_{1}, \ldots, A_{m}$ is called the antecedent of the sequent and $B_{1}, \ldots, B_{n}$ is called the succedent of the sequent. The meaning of

$$
A_{1}, \ldots, A_{m} \rightarrow B_{1}, \ldots, B_{n} \text { is } A_{1} \wedge \cdots \wedge A_{m} \rightarrow B_{1} \vee \cdots \vee B_{n} .
$$

We use Greek capital letters $\Gamma, \Pi, \Delta, \Lambda, \Gamma_{0}, \Gamma_{1}, \ldots$ to denote finite (possibly empty) sequences of formulas separated by commas.

Definition 3.1.4. An inference is an expression of the form

$$
\frac{S_{1}}{S} \text { or } \frac{S_{1} S_{2}}{S}
$$

where $S_{1}, S_{2}$ and $S$ are sequents. $S_{1}$ and $S_{2}$ are called the upper sequents and $S$ is called the lower sequent of the inference.

Intuitively this means that when $S_{1}\left(S_{1}\right.$ and $\left.S_{2}\right)$ is (are) asserted, we can infer $S$ from it (from them). We restrict ourselves to inferences obtained from the following rules of inferences, in which $A, B, C, D, F(\alpha)$ denotes formulas.

1) Structural rules:

1.1) Weakening:

$$
\text { left: } \frac{\Gamma \rightarrow \Delta}{D, \Gamma \rightarrow \Delta} ; \text { right: } \frac{\Gamma \rightarrow \Delta}{\Gamma \rightarrow \Delta, D} .
$$

The formula $D$ is called the weakening formula.

1.2) Contraction:

$$
\text { left: } \frac{D, D, \Gamma \rightarrow \Delta}{D, \Gamma \rightarrow \Delta} ; \text { right: } \frac{\Gamma \rightarrow \Delta, D, D}{\Gamma \rightarrow \Delta, D} .
$$

1.3) Exchange:

$$
\text { left: } \frac{\Gamma, C, D, \Pi \rightarrow \Delta}{\Gamma, D, C, \Pi \rightarrow \Delta} \text {; right: } \frac{\Gamma \rightarrow \Delta, C, D, \Lambda}{\Gamma \rightarrow \Delta, D, C, \Lambda} .
$$

1.4) Cut:

$$
\frac{\Gamma \rightarrow \Delta, D \quad D, \Pi \rightarrow \Lambda}{\Gamma, \Pi \rightarrow \Delta, \Lambda} .
$$

The formula $D$ is called the cut formula of this inference.

2) Logical rules:

2.1)

$$
\neg \text { left: } \frac{\Gamma \rightarrow \Delta, D}{\neg D, \Gamma \rightarrow \Delta} ; \neg \text { right: } \frac{D, \Gamma \rightarrow \Delta}{\Gamma \rightarrow \Delta, \neg D} .
$$

The formulas $D$ and $\neg D$ are called the auxiliary formula and the principal formula, respectively, of this inference. 
2.2)

$$
\begin{aligned}
& \wedge \text { left: } \frac{C, \Gamma \rightarrow \Delta}{C \wedge D, \Gamma \rightarrow \Delta} \text { and } \frac{D, \Gamma \rightarrow \Delta}{C \wedge D, \Gamma \rightarrow \Delta} ; \\
& \wedge \text { right: } \frac{\Gamma \rightarrow \Delta, C \Gamma \rightarrow \Delta, D}{\Gamma \rightarrow \Delta, C \wedge D}
\end{aligned}
$$

The formulas $C$ and $D$ are called auxiliary formulas and $C \wedge D$ is called the principal formula of this inference.

2.3)

$$
\forall \text { left: } \frac{F(V), \Gamma \rightarrow \Delta}{\forall \varphi F(\varphi), \Gamma \rightarrow \Delta} ; \forall \text { right: } \frac{\Gamma \rightarrow \Delta, F(\alpha)}{\Gamma \rightarrow \Delta, \forall \varphi F(\varphi)} .
$$

Where $V$ is an arbitrary abstract with the same type as $\varphi$ and $\alpha$ does not occur in the lower sequent. The formulas $F(V)$ and $F(\alpha)$ are called auxiliary formulas and $\forall \varphi F(\varphi)$ the principal formula. The $\alpha$ in $\forall$ : right is called the eigenvariable of this inference. Note that $V, \varphi, \alpha$ may be of type 0 , that is, they may be of the form $t, x, a$ respectively. Note also that every abstract $V$ is arithmetical. ater.

$\forall$ left is equivalent to the arithmetical comprehension axioms which we prove

Definition 3.1.5 (Formal proofs).

A proof $P$ is a tree of finite sequents satisfying the following conditions:

1) The topmost sequents of $P$ are of the form

$$
D \rightarrow D
$$

where $D$ is an atomic formula.

2) Every sequent of $P$ except the lowest one is an upper sequent of an inference whose lower sequent is also in $P$.

A topmost sequent of $P$ is called an initial sequent. The unique lowest sequent of a proof $P$ will be called the end-sequent of $P$. A proof with end-sequent $S$ is called a proof of $S$.

Let us name our (logical) system LS. A sequent $S$ is called provable (or provable in $L S$ ) if there is a proof of it.

Theorem 3.1.1. For every formula $D$,

$$
D \rightarrow D
$$

is provable.

Proof. Immediate by the number of logical symbols in $D$. 
Theorem 3.1.2 (Cut elimination Theorem).

If a sequent $S$ is provable, then $S$ is also provable without using cuts.

Proof. Easily obtained from Gentzen's original proof of his Hauptsatz by defining the grade of the formula $A$ by the following equation:

$$
\text { the grade of } A=\omega \cdot a+b
$$

where $a$ is the number of higher type quantifiers in $A$ and $b$ is the number of logical symbols in $A$. See PT for details.

We use $\vee, \supset, \equiv, \exists$ as combinations of $\neg, \wedge, \forall$.

Here we give two example of inferences which will be used quite often.

1.

$$
\frac{A(0) \wedge \forall x(A(x) \supset A(x+1)) \supset \forall x A(x), \Gamma \rightarrow \Delta}{\forall \varphi(\varphi[0] \wedge \forall x(\varphi[x] \supset \varphi[x+1]) \supset \forall x \varphi[x]), \Gamma \rightarrow \Delta}
$$

where $A(0)$ is arithmetical, that is, it has no higher type quantifiers.

2 .

$$
\frac{\rightarrow \forall x(A(x) \equiv A(x))}{\rightarrow \exists \varphi \forall x(\varphi[x] \equiv A(x))}
$$

where $A(x)$ is arithmetical, that is, it has no higher type quantifiers. Note that this inference is a combination of $\neg$ left, $\forall$ left, and $\neg$ right. Formulas of this form are called arithmetical comprehension axioms. These are equivalent to $\forall$ left inferences.

Definition 3.1.6 (Mathematical axioms).

The set of mathematical axioms consist of the following sentences.

1. First order Peano axioms on $0,1,+, \cdot,<,=$.

First order axioms of mathematical induction are excluded here since they are included in a higher type form.

2. Mathematical induction (MI).

$$
\forall \varphi(\varphi[0] \wedge \forall x(\varphi[x] \supset \varphi[x+1]) \supset \forall x \varphi[x])
$$

3. Equality axiom (EQ).

$$
\forall \varphi \forall \forall y(x=y \wedge \varphi[x] \supset \varphi[y]) .
$$

The set of these sentences will be denoted by $\Gamma_{0}$. The first system of finite type arithmetic, $\mathrm{FA}_{1}$, is the system with the logical system LS and the mathematical axioms of $\Gamma_{0}$, that is, $\Gamma \rightarrow \Delta$ is provable in $\mathrm{FA}_{1}$ iff $\Gamma, \Gamma_{0} \rightarrow \Delta$ is provable in LS. Also $A$ is provable in $\mathrm{FA}_{1}$ iff $\Gamma_{0} \rightarrow A$ is provable in LS.

Theorem 3.1.3. Our first system $F A_{1}$ is a conservative extension of (first order) Peano arithmetic. 
Proof. (Cf. Problem 16. 10. in PT) Let $A$ be a first order formula. Suppose that $A$ is provable in $\mathrm{FA}_{1}$. By the cut elimination theorem, there exists a cutfree proof $P$ of

$$
\Gamma_{0} \rightarrow A
$$

Observe the following.

1) There is no higher type $\forall$ right in $P$ since $A$ does not have any higher type quantifiers. Higher type quantifiers in $\Gamma_{0}$ should be introduced by $\forall$ left, and therefore higher type quantifiers introduced by $\forall$ right cannot disappear without cuts.

2) In the same way as in 1), all higher type quantifiers introduced by $\forall$ left are $\forall \varphi$ the outermost logical symbol in MI or EQ.

3) Because of 1), one can replace all higher type free variables by

$$
0=0
$$

4) By 2) and 3), we can replace

$$
\frac{A(0) \wedge \forall x(A(x) \supset A(x+1)) \supset \forall x A(x), \Gamma \rightarrow \Delta}{\forall \varphi(\varphi[0] \wedge \forall x(\varphi[x] \supset \varphi[x+1]) \supset \forall x \varphi[x]), \Gamma \rightarrow \Delta}
$$

and

$$
\frac{\forall x \forall y(A(x) \wedge x=y \supset A(y)), \Gamma \rightarrow \Delta}{\forall \varphi \forall x \forall y(\varphi[x] \wedge x=y \supset \varphi[y]), \Gamma \rightarrow \Delta}
$$

by

$$
\frac{A^{\prime}(0) \wedge \forall x\left(A^{\prime}(x) \supset A^{\prime}(x+1)\right) \supset \forall x A^{\prime}(x), \Gamma \rightarrow \Delta}{\overline{\forall z_{1} \cdots \forall z_{m}\left(A^{\prime}(0) \wedge \forall x\left(A^{\prime}(x) \supset A^{\prime}(x+1)\right) \supset \forall x A^{\prime}(x)\right), \Gamma \rightarrow \Delta}}
$$

and

$$
\frac{\forall x \forall y\left(A^{\prime}(x) \wedge x=y \supset A^{\prime}(y)\right), \Gamma \rightarrow \Delta}{\overline{\forall z_{1} \cdots \forall z_{m} \forall x \forall y\left(A^{\prime}(x) \wedge x=y \supset A^{\prime}(y)\right), \Gamma \rightarrow \Delta}}
$$

where two lines between the upper sequent and the lower sequent indicate several inferences and $\forall z_{1} \cdots \forall z_{m}$ quantifies all free variables in $A^{\prime}(0)$. Then MI and EQ become finitely many instances of first order mathematical induction and the first order equality axioms. Therefore the theorem is proved.

The system $\mathrm{FA}_{1}$ is the one in which we will develop classical analysis.

Theorem 3.1.4. Let $A(a)$ be a formula, possibly with higher type quantifiers. Then

$$
\forall x \forall y(A(x) \wedge x=y \supset A(y))
$$

is $F A_{1}$-provable. 
Proof. Immediate by mathematical induction on the number of logical symbols in $A(a)$.

Note that the analogy of Theorem 3.1.4 for MI is not possible. This is easily seen as follows. If such an analogy can be proved, then by $\S 18$. in PT, the truth definition of the first order Peano arithmetic can be established in $\mathrm{FA}_{1}$ which contradicts Theorem 3.1.3.

In our original lecture notes, we used a different system from $\mathrm{FA}_{1}$. We would like to explain the situation here. We denote the logical system in the lecture notes by and the mathematical system there by $\mathrm{FA}_{1}^{\prime}$. LN is obtained from LS by restricting $\forall$ left in the following way. Which makes LN weaker than LS.

$\forall$ left in LN

$$
\frac{F(V), \Gamma \rightarrow \Delta}{\forall \varphi F(\varphi), \Gamma \rightarrow \Delta} \text { or } \frac{F(\alpha), \Gamma \rightarrow \Delta}{\forall \varphi F(\varphi), \Gamma \rightarrow \Delta}
$$

where $V$ does not have higher type free variables. Through LN is weaker than $\mathrm{LS}$, mathematical axioms in $\mathrm{FA}_{1}^{\prime}$ is stronger than the mathematical axioms in $\mathrm{FA}_{1}$.

Definition 3.1.7. Mathematical axioms in $F A_{1}^{\prime}$. The set of mathematical axioms consist of the following formulas.

1. Peano axioms on $0,1,+, \cdot,<,=$.

2. Mathematical induction (MI') in the strongest form, viz., for any formula of our language, say $A(a)$, the following is an instance of $M I^{\prime}$ ':

$$
\forall Y_{1} \cdots \forall Y_{m}\left(A^{\prime}(0) \wedge \forall x\left(A^{\prime}(x) \supset A^{\prime}\left(x^{\prime}\right)\right) \supset \forall x A^{\prime}(x)\right),
$$

where $A^{\prime}(0)$ is obtained from $A(0)$ by replacing all the free variables by distinct bound variables $Y_{1}, \ldots, Y_{m}$ (hence $Y_{1}, \ldots, Y_{m}$ are not necessarily of type 0).

3. Equality axiom (EQ) is introduced in the following form: for every $A(a)$, $\forall Y_{1} \cdots \forall Y_{m} \forall x \forall y\left(x=y \supset\left(A^{\prime}(x) \equiv A^{\prime}(y)\right)\right)$, where $A^{\prime}$ and $Y_{1}, \ldots, Y_{m}$ have the same meaning as in 2.

The set of these formulas will be called $\Gamma_{0}^{\prime}$. The system $\mathrm{FA}_{1}^{\prime}$ is the one with the logical system LN and the mathematical axioms of $\Gamma_{0}^{\prime}$. Note that a formula $A$ (of our language) is provable in $\mathrm{FA}_{1}^{\prime}$ if and only if $\Gamma \rightarrow A$ is provable in LN, where $\Gamma$ is a finite subset of $\Gamma_{0}^{\prime}$. Therefore we identify those two statements. Also, we write $\Gamma_{0}^{\prime} \rightarrow A$ in order to express that $\Gamma \rightarrow A$ is provable for some finite $\Gamma$.

Theorem 3.1.5. $F A_{1}^{\prime}$ is a conservative extension of (first order) Peano arithmetic. 
Proof. Let $A$ be an arithmetical formula (without any higher type variable). Suppose $\Gamma_{0}^{\prime} \rightarrow A$ is provable (in $\mathrm{LN}$ ). We wish to show that $\rightarrow A$ is provable in Peano arithmetic.

Let $\tau$ be the type of a quantifier which occurs in the proof. Consider all the uses of $\forall$ left of type $\tau$ in the proof and let those abstracts be $V_{1}, \ldots, V_{n}$. In order to simplify the notation, we write each of those as $V_{j}(a):\left\{\varphi_{1}, \ldots, \varphi_{i}\right\} A\left(\varphi_{1}, \ldots, \varphi_{i}, a\right)$, where $a$ represents all the free variables of type 0 in $V_{j}$.

Case 1) $n=0$. Consider $\left\{\varphi_{1}, \ldots, \varphi_{i}\right\}(0=0)$. This is the "type $\tau$ version" of $0=0$, which we may call $U$ temporarily. We will replace each variable of type $\tau$ by $U$ and knock off excessive inferences throughout the proof.

Such replacement is defined by induction on the number of logical symbols in a formula or abstract. A free variable of type $\tau$, say $\alpha^{\tau}$ occurs either in the form $\alpha^{\tau}[\cdots]$ or $\alpha\left[\cdots \alpha^{\tau}, \cdots\right]$. In the first case, replace the entire part by $0=0$. In the latter, replace $\alpha^{\tau}$ by $U$. For other formulas, the replacement is defined from the induction hypothesis. For example, if $F(\varphi)$ has been converted to $F^{\prime}(\varphi)$ and $\varphi$ is not of type $\tau$, then $\forall \varphi F(\varphi)$ is transformed to $\forall \varphi F^{\prime}(\varphi)$. If $F(\varphi)$ has been converted to $F^{\prime}(\varphi)$ and $\varphi$ is of type $\tau$, then $\forall \varphi F(\varphi)$ is transformed to $F^{\prime}(U)$.

It is obvious that initial sequents are converted to the initial sequents: In $\forall$ right, say $\frac{\Gamma \rightarrow \Delta, F\left(\alpha^{\tau}\right)}{\Gamma \rightarrow \Delta, \forall \varphi F(\varphi)}$,

the upper sequent has turned to $\Gamma^{\prime} \rightarrow \Delta^{\prime}, F^{\prime}(U)$. Eliminate this inference. We treat $\forall$ left in a similar manner. Consider a use of $\forall$ left whose abstract is not of type $\tau$ :

$$
\frac{F(V), \Gamma \rightarrow \Delta}{\forall \varphi F(\varphi): \Gamma \rightarrow \Delta}
$$

where the type of $V$ is not $\tau$. Recall that $V$ contains no higher order free variables. This part is transformed to

$$
\frac{F^{\prime}(V), \Gamma^{\prime} \rightarrow \Delta^{\prime}}{\forall \varphi F^{\prime}(\varphi), \Gamma^{\prime} \rightarrow \Delta^{\prime}}
$$

which is another $\forall$ left.

The endsequent does not contain higher order free variables, hence remains unchanged through the process.

Case 2) $n>0$. For this case we first define a transformation of formulas in $P$ in a manner that the resulting formulas are rid of quantifiers of type $\tau$. Let $\forall \varphi F(\varphi)$ be a formula where $\varphi$ is of type $\tau$. Then change $\forall \varphi F(\varphi)$ to $\forall x F^{\prime}\left(V_{1}(x)\right) \wedge \forall x F^{\prime}\left(V_{2}(x)\right) \wedge \cdots \wedge \forall x F^{\prime}\left(V_{n}(x)\right)$, where $F^{\prime}$ is the transformation of $F$, which has already been defined. The transformation of an arbitrary formula should be obvious.

Next we prove the following statement:

$\left({ }^{*}\right)$ Given a sequent in $P$, say

$$
\Gamma\left(\alpha_{1}, \ldots, \alpha_{m}\right) \rightarrow \Delta\left(\alpha_{1}, \ldots, \alpha_{m}\right),
$$


where $\alpha_{1}, \ldots, \alpha_{m}$ are all the free variables in the sequent whose type is $\tau$, we can construct a proof of a sequent

$$
\Gamma^{\prime}\left(V_{i_{1}}\left(a_{1}\right), \ldots, V_{i_{m}}\left(a_{m}\right)\right) \rightarrow \Delta^{\prime}\left(V_{i_{1}}\left(a_{1}\right), \ldots, V_{i_{m}}\left(a_{m}\right)\right)
$$

where $V_{i_{j}}$ is any one of $V_{1}, \ldots, V_{n}$, where $a_{1}, \ldots, a_{m}$ are new, mutually distinct free variables, $\Gamma^{\prime}$ and $\Delta^{\prime}$ are obtained from $\Gamma$ and $\Delta$ respectively by replacing all the quantifiers of type $\tau$ by those of first order in a manner as described above, and the proof of this sequent does not contain any quantifier of type $\tau$.

Notice that there can be $n^{m}$ of those sequents.

$1^{\circ}$. Initial sequents. Suppose $S\left(\alpha_{1}, \ldots, \alpha_{m}\right)$ is an initial sequent in $P$ and $\alpha_{1}, \ldots, \alpha_{m}$ are all the free variables of type $\tau$ in $S$. Then $S$ is transformed to $S^{\prime}$ by the first transformation. $S^{\prime}\left(V_{i_{1}}\left(a_{1}\right), \ldots, V_{i_{m}}\left(a_{m}\right)\right)$ is a sequent of the form $D \rightarrow D$ which does not contain any quantifier of type $\tau$. So it is provable without any quantifiers of type $\tau$.

$2^{\circ} . \forall$ left of type $\tau$ of the form $\frac{F\left(V_{i}(a)\right), \Gamma \rightarrow \Delta}{\forall \varphi F(\varphi), \Gamma \rightarrow \Delta}$.

Let us denote the upper sequent by $F\left(V_{i}(a), \alpha_{1}, \ldots, \alpha_{m}\right), \Gamma\left(\alpha_{1}, \ldots, \alpha_{m}\right) \rightarrow$ $\Delta\left(\alpha_{1}, \ldots, \alpha_{m}\right)$. It has been changed to:

$$
F^{\prime}\left(V_{i}(a), V_{i_{1}}\left(a_{1}\right), \ldots, V_{i_{m}}\left(a_{m}\right)\right), \Gamma^{\prime}(\cdots) \rightarrow \Delta^{\prime}(\cdots) .
$$

By adding some logical inferences, we can deduce:

$$
\begin{gathered}
\forall x F^{\prime}\left(V_{1}(x), V_{i_{1}}\left(a_{1}\right), \ldots, V_{i_{m}}\left(a_{m}\right)\right) \wedge \cdots \\
\wedge \forall x F^{\prime}\left(V_{n}(x), V_{i_{1}}\left(a_{1}\right), \ldots, V_{i_{m}}\left(a_{m}\right)\right), \Gamma^{\prime}(\cdots) \rightarrow \Delta^{\prime}(\cdots) .
\end{gathered}
$$

$3^{\circ} . \forall$ left of type $\tau$ of the form $\frac{F\left(\alpha_{k}\right), \Gamma \rightarrow \Delta}{\forall \varphi F(\varphi), \Gamma \rightarrow \Delta}$.

The upper sequent can be transformed to:

$$
F^{\prime}\left(V_{i_{k}}\left(a_{k}\right), V_{i_{1}}\left(a_{1}\right), \ldots, V_{i_{m}}\left(a_{m}\right)\right), \Gamma^{\prime}(\cdots) \rightarrow \Delta^{\prime}(\cdots),
$$

for any given $V_{i_{1}}\left(a_{1}\right), \ldots, V_{i_{k}}\left(a_{k}\right), \ldots, V_{i_{n}}\left(a_{m}\right)$. By adding some logical inferences, we obtain

$$
\begin{gathered}
\forall x F^{\prime}\left(V_{1}(x), V_{i_{1}}\left(a_{1}\right), \cdots, V_{i_{m}}\left(a_{m}\right)\right) \wedge \cdots \\
\wedge \forall x F^{\prime}\left(V_{n}(x), V_{i_{1}}\left(a_{1}\right), \ldots, V_{i_{m}}\left(a_{m}\right)\right), \Gamma^{\prime}(\cdots) \rightarrow \Delta^{\prime}(\cdots) .
\end{gathered}
$$

$4^{\circ} . \forall$ right of type $\tau: \frac{\Gamma \rightarrow \Delta, F\left(\alpha_{k}\right)}{\Gamma \rightarrow \Delta, \forall \varphi F(\varphi)}$.

Let $\tilde{\alpha}$ be the sequence of free variables in the lower sequent, say $\alpha_{1}, \ldots, \alpha_{m}$, where $\alpha_{k}$ is missing. Let us denote the upper sequent by $\Gamma(\tilde{\alpha}) \rightarrow \Delta(\tilde{\alpha}), F\left(\alpha_{k}, \tilde{\alpha}\right)$ and let us denote the sequence of abstracts $V_{1}\left(a_{1}\right), \ldots, V_{m}\left(a_{m}\right)$ where $V_{k}$ 
is missing by $\tilde{V}$. Consider any number $i_{k}$ such that $1 \leq i_{k} \leq n$. Then we have $\Gamma^{\prime}(\tilde{V}) \rightarrow \Delta^{\prime}(\tilde{V}), F^{\prime}\left(V_{i_{k}}\left(a_{k}\right), \tilde{V}\right)$, from which we obtain

$$
\Gamma^{\prime}(\tilde{V}) \rightarrow \Delta^{\prime}(\tilde{V}), \forall x F^{\prime}\left(V_{i_{k}}(x), \tilde{V}\right) .
$$

Since $i_{k}$ is arbitrary, it follows that

$$
\Gamma^{\prime}(\tilde{V}) \rightarrow \Delta^{\prime}(\tilde{V}), \forall x F^{\prime}\left(V_{1}(x), \tilde{V}\right) \wedge \cdots \wedge \forall x F^{\prime}\left(V_{n}(x), \tilde{V}\right) .
$$

$5^{\circ} . \forall$ left whose type is not $\tau: \frac{F(U), \Gamma \rightarrow \Delta}{\forall \varphi F(\varphi), \Gamma \rightarrow \Delta}$.

The upper sequent has been transformed to:

$$
F^{\prime}\left(U, V_{i_{1}}\left(a_{1}\right), \ldots\right), \Gamma^{\prime}(\cdots) \rightarrow \Delta^{\prime}(\cdots) .
$$

So we obtain

$$
\forall \varphi F^{\prime}\left(\varphi, V_{i_{1}}\left(a_{1}\right), \ldots\right), \Gamma^{\prime}(\cdots) \rightarrow \Delta^{\prime}(\cdots) .
$$

Other cases can be treated similarly.

Thus, one by one, we can eliminate quantifiers of higher type and in the end we obtain a first order proof of $\Gamma_{0}^{\prime \prime} \rightarrow A$, where $\Gamma_{0}^{\prime \prime}$ does not contain any higher order variables. Each formula of $\Gamma_{0}^{\prime \prime}$ is an instance of an axiom of Peano arithmetic, hence $A$ is provable in Peano arithmetic.

(We must of course see that $\Gamma_{0}^{\prime \prime}$ consist of first order instances of $\Gamma_{0}^{\prime}$. Suppose, for instance, $\forall \varphi F(\varphi)$ is an induction formula. This part is transformed to $\forall x F\left(V_{1}(x), a\right) \wedge \cdots \wedge \forall x F\left(V_{n}(x), a\right)$, which can still serve as an induction formula.)

As I explained immediately after Theorem 3.1.4, the union of $\mathrm{FA}_{1}$ and $\mathrm{FA}_{1}^{\prime}$ is not a conservative extension of Peano arithmetic.

In my lecture notes, I actually carried out everything in $\mathrm{FA}_{1}$ but pretended that I did so in $\mathrm{FA}_{1}^{\prime}$. Let me explain it here.

Suppose that the following inference is an inference in LS but not an inference in $\mathrm{LN}$.

$$
\frac{F\left(V\left(\alpha_{1}, \ldots, \alpha_{n}\right)\right), \Gamma \rightarrow \Delta}{\exists \chi F(\chi), \Gamma \rightarrow \Delta}
$$

where $\alpha_{1}, \ldots, \alpha_{n}$ are all higher type free variables in $V\left(\alpha_{1}, \ldots, \alpha_{n}\right)$. Then the following is an inference in LN.

$$
\frac{F\left(V\left(\alpha_{1}, \ldots, \alpha_{n}\right)\right), \Gamma \rightarrow \Delta}{\exists \chi F\left(\chi\left(\alpha_{1}, \ldots, \alpha_{n}\right)\right), \Gamma \rightarrow \Delta} .
$$

Of course, $\exists \chi F\left(\chi\left(\alpha_{1}, \ldots, \alpha_{n}\right)\right)$ and $\exists \chi F(\chi)$ are different but have the same mathematical interpretation. Therefore one can claim that one is doing in $\mathrm{FA}_{1}^{\prime}$ when he is actually doing in $\mathrm{FA}_{1}$ since he is only skipping some transformation and the result would be the same for an arithmetical sentence. However in 
practice this transformation is extremely complicated as is easily seen in the following example. Suppose we proved the following formula in $\mathrm{FA}_{1}^{\prime}$

$$
\forall \xi F(\alpha, \beta, \xi(\alpha, \beta)) .
$$

In order to apply this theorem for some special case, we have to change this theorem to the form

$$
\forall \xi F(\alpha, \beta, \xi(\alpha, \beta, \gamma)) .
$$

In general, we should prove infinitely many instances of the form

$$
\forall \xi F\left(\alpha, \beta, \xi\left(\alpha, \beta, \gamma_{1}, \gamma_{2}, \ldots\right)\right) .
$$

The reason that I pretended to take the complicated system $\mathrm{FA}_{1}^{\prime}$ in the place of $\mathrm{FA}_{1}$ is the following. Kreisel strongly insisted that one should take the strongest form of mathematical induction, that is, $\mathrm{MI}^{\prime}$ in $\mathrm{FA}_{1}^{\prime}$. This made me believe that there would be some important theorem where $\mathrm{MI}^{\prime}$ would be essential and that the complication in $\mathrm{FA}_{1}^{\prime}$ might be worthwhile. However in my case study, I have not encountered any such case and I am returning to my original system. Besides, $\mathrm{FA}_{1}$ would be much better for producing (nonprimitive) recursive functions by interpreting the theorems in analysis. If we try to build a measure theory in our framework, then the situation seems to be different. Consider for example hypothesis of the form

$$
\forall n \text { ( } X_{n} \text { is measurable) }
$$

where $X_{n}$ is an arithmetically defined sequence of sets of reals. We cannot in general find an arithmetically defined sequence $M_{n}$ of measures of $X_{n}$. Then we use instead the hypothesis of measured sequence of sets i.e. a pair of sequences $\left(X_{n}, M_{n}\right)$ and the hypothesis

$$
\forall n\left(M_{n} \text { is the measure of } X_{n}\right) .
$$

Now we have to do with measured sequences whenever we speak of measurable sequences in the usual texts. Since $\left(M_{n}\right.$ is the measure of $\left.X_{n}\right)$ is quite a complicated predicate, we have to use the whole induction in this situation. See [10] and [11] for this matter.

There is also a temptation to use a stronger system than $\mathrm{FA}_{1}$ provided that the system is a conservative extension of Peano arithmetic. However in a sense, if the system is weaker, the result is better. And also $\mathrm{FA}_{1}$ is the system most likely to have a good interpretation for our program to produce mathematical (nonprimitive recursive functions).

\subsection{The second system}

Our first system, $\mathrm{FA}_{1}$, is not convenient in practice when we wish to develop analysis, hence the introduction of the second system, FA, which is equivalent to $\mathrm{FA}_{1}$ but formulated in a slightly different way. It is, in its mathematical context, the system of rational numbers. 
Definition 3.2.1. In addition to the symbols of $F A_{1}, F A$ has the following as primitive symbols (constants).

Unary predicate : $N$ :

Binary predicate $: \leq$.

The intended meanings of those constants are: $N(a)$

means "a is a natural number" and $a \leq b$ means " $a$ is equal to or less than $b$ ".

Definition 3.2.2. The logical system for FA is LS defined in $\S 3.1$. The mathematical axioms for FA, say $\tilde{\Gamma}$, are essentially those of Peano's for natural numbers and the axioms on rationals

1. $N(0) ; \forall x(N(x) \supset N(x+1))$

2. Eq: Equality axioms.

3. MI: Mathematical induction is formulated in the form:

$$
\forall \varphi(\varphi[0] \wedge \forall x(N(x) \wedge \varphi[x] \supset \varphi[x+1]) \supset \forall x(N(x) \supset \varphi[x])) .
$$

4. Axioms on $0,1,+$ and $\cdot$, relativized to $N$.

$\forall x(N(x) \wedge N(y) \supset x+(y+1)=(x+y)+1)$ is such an axiom.

5. Axioms on,,$+- \cdot$ and $\div$ (for rationals as a field).

Examples. $\forall x \forall y(x+y=y+x)$;

$$
\forall x \forall y \forall z(y \neq 0 \supset(x / y=z \equiv x=y z)) .
$$

6. Relating $N$ to rationals

$$
\forall x \exists y \exists z(0<x \supset N(y) \wedge N(z) \wedge z \neq 0 \wedge x=y / z) .
$$

It can be easily shown that the axioms of the second system are interpretable in the first system, hence the second system is also a conservative extension of Peano arithmetic. (Define rationals as the equivalence classes of pairs of natural numbers, using a pairing function.)

It is in this system, FA, we are going to carry our analysis.

Remark. For the proof of the fact that FA is a conservative extension of Peano arithmetic, we would like to add the following explanation. First make a standard interpretation of $\mathrm{FA}$ in $\mathrm{FA}_{1}$. Then let $A$ be a first order formula in $F A_{1}$. It is easily seen that

$$
A \equiv A^{N}
$$

is provable in $\mathrm{FA}_{1}$ by mathematical induction on the number of logical symbols in $A$, where $A^{N}$ is obtained from $A$ by replacing $\forall x$ by $\forall x(N(x) \supset)$. From this follows the theorem that FA is a conservative extension of Peano arithmetic. 


\subsection{Defining reals}

Our object is to develop a theory of classical analysis using only arithmetical comprehension axioms. Thus this theory can be formulated in our system FA.

We will not develop the whole theory in a strictly formal manner. Rather, we will present a way of doing calculus, only pointing out how to avoid nonarithmetical comprehension. We shall first introduce some informal notations, which are familiar to the working mathematicians. For example, we will identify $\wedge$ (and) and $\cap$ (intersection). We will use $A=\{x \mid A(x)\}$ to mean, as usual, the set of $x$ (rationals) which satisfy $A(x)$. Then $A(x)$ may be denoted by $x \in A$. The set $A$, as above, can be identified with the abstract $\{x\} A(x)$. The intersection $A \cap B$ may be used for $\{x\}(A(x) \wedge B(x))$. Similarly with $\cup$. An abstract such as $\{x\}(\exists y A(y) \wedge B(x, y))$ may be expressed as $\bigcup_{y \in A}\{x \mid B(x, y)\}$. We do not attempt to list all the possible notation; the context of each notation will appeal to common sense.

A formula $A$ is said to be arithmetical if the quantifiers in $A$ are of type 0. Note that all abstracts are arithmetical by definition.

Let $\alpha$ be of type 1 . We can identify $\alpha$ with $\{x\} \alpha(x)$, that is, $\{x \mid \alpha(x)\}$. In this sense, our discussion of abstracts always includes a discussion of free variables as a special case.

Definition 3.3.1. Reals. Let $\alpha$ be a free variable of type 1 . We will use $R(\alpha)$ to denote the following formula:

$$
\exists x \alpha[x] \wedge \exists x \neg \alpha[x] \wedge \forall x(\alpha[x] \equiv \exists y(x<y \wedge \alpha[y])) .
$$

The intended meaning of $R(\alpha)$ is of course that $\alpha$ is a Dedkind cut of rationals, or $\alpha$ is a real number.

Hence, if $R(A)$, then we say that $A$ is a real number.

Notice that $R$ is arithmetical.

Proposition 3.3.1. Rationals among reals are definable as abstracts from original rationals.

Proof. Let $a$ be any term (a formal expression of type 0). Then there is an abstract of type 1 , say $\hat{a}$, such that $\forall x(\hat{a}(x) \equiv x<a)$. Namely $\hat{a}$ is $\{y\}(y<a)$. This defines $a$ as a Dedekind cut. Let $a$ be any rational number. Then the proposition is obvious.

Remark. A real number $\alpha$ is said to be implicitly defined by $A$ if $\exists ! \varphi A(\varphi)$ (there is a unique $\varphi$ such that $A(\varphi))$ and $A(\alpha)$. In impredicative mathematics, the existence of a set such as $\{x \mid \exists \varphi(A(\varphi) \wedge \varphi(x))\}$ can be established and in the presence of $\exists ! \varphi A(\varphi)$, the equivalence of $\alpha$ and $\{x\}(\exists \varphi(A(\varphi) \wedge \varphi(x)))$ can be proved. Hence $\alpha$ is explicitly definable (not by an arithmetical abstract). So implicit definability and explicit definability amount to the same. In our system, on the other hand, we cannot establish the existence of $\{x \mid \exists \varphi(A(\varphi) \wedge \varphi(x))\}$ (an impredicative set), hence arises the importance of the difference between implicit and explicit definabilities. 
Proposition 3.3.2. The basic relations of reals, $=$ and $<$, can be expressed arithmetically. The four arithmetic operations of reals,,,$+- \cdot$ and $\div$, can be defined by abstracts.

Proof. 1) Let $E(\alpha, \beta)$ denote: $\forall x(\alpha[x] \equiv \beta[x])$. Then define equality (=) to be $\{\varphi, \psi\} E(\varphi, \psi)$. We denote $E(\alpha, \beta)$ by $\alpha=\beta$.

2) Let $I(\alpha, \beta)$ denote $\exists x(\neg \alpha[x] \wedge \beta[x])$. Then $I(\alpha, \beta)$ expresses $\alpha<\beta$.

Of course $=$ and $<$ defined as above make sense only when $R(\alpha)$ and $R(\beta)$ are assumed.

3) Let $A(\alpha, \beta, a)$ denote $\exists x \exists y(\alpha[x] \wedge \beta[y] \wedge a=x+y)$. Then + is defined to be $\{\varphi, \psi, z\} A(\varphi, \psi, z)$. We write $\alpha+\beta$ for $\{z\} A(\alpha, \beta, z)$.

Other operations will be dealt with in a later section when we introduce the precise definition of function. The basic properties for those relations and operations can be easily proved (though tedious) and the proof can be carried out in FA. Try $R(\alpha) \wedge R(\beta) \rightarrow R(\alpha+\beta)$.

Remark. The completeness of the reals, in its absolute sense, is not provable in our system. A weak form of completeness is, however, provable, namely, a sequence of reals which has an upper bound has a definable least upper bound. The meaning of this will become clear later.

From now on, we will make it "look like calculus", applying standard notation from calculus as much as possible, hence a drastic change must be made in the use of some letters. Also some abbreviated notation will be introduced.

Let $a, b, c, x, y, z, \ldots$ stand for reals, while $r, s, t, \ldots, \varepsilon, \delta, \ldots$ stand for rationals. In the usual $\varepsilon-\delta$ method, we may think that $\varepsilon$ and $\delta$ run over the rational number. Thus $\forall x A(x)$ will be an abbreviation of $\forall \varphi(R(\varphi) \supset A(\varphi))$. If $x$ stands for a real and $r$ stands for a rational, an expression such as $x>r$ is an "abbreviated" expression for $x>\hat{r}$, where $\hat{r}$ is $\{x\}(t<r)$.

An expression such as $\forall r A(\hat{r})$ can be a legitimate formula: Let $A(\hat{r})$ be $A(\{y\}(y<r))$, which is a formula. Then $\forall r A(\{y\}(y<r))$, or $\forall r A(\hat{r})$ is a formula.

The following are some useful facts.

Proposition 3.3.3. 1) $\forall \varphi \forall r(R(\varphi) \supset(r<\varphi \equiv \varphi[r]))$;

2) $\forall x \forall y(x<y \supset \exists r(x<r<y))$; and

3) $\forall x \forall y(x<y \supset \exists r \exists s(x<r<s<y))$ are provable in FA, where $x$ and $y$ stand for reals and $r$ and $s$ stand for rationals.

$\phi$ (the empty set) denotes $\{r\}(r<r)$. 


\subsection{Functions}

We will define functions of reals in some intervals. Let me list again our convention that $\Gamma(a, b, \ldots) \rightarrow \Delta(a, b, \ldots)$ means $R(\alpha), R(\beta), \ldots, \Gamma(\alpha, \beta, \ldots) \rightarrow$ $\Delta(\alpha, \beta, \ldots)$.

Proposition 3.4.1. Intervals of reals (closed, open, half closed, bounded, unbounded) are explicitly definable in parameters. We use the standard notation such as $[a, b]$ and $c \in[a, b]$.

Proof. The interval $[a, b]$ is $\{\varphi\}(R(\varphi) \wedge a \leq \varphi \leq b)$ and $c \in[a, b]$ means $a \leq c \leq b$. An interval is definable if the end points are.

Definition 3.4.1. An abstract $\{r\} A(r, c)$, where $c$ is a free variable of type 1 and there may be some parameters in $A$, is said to define a function in the interval $I$ explicitly (in these parameters) if $c \in I \rightarrow R(\{r\} A(r, c))$ is provable in $F A$. Or we do the discussion under the assumption that $\forall c \in I(R(\{r\} A(r, c)))$. A function defined by $\{r\} A(r, c)$ as above may be expressed as $f(c)=\{r \mid A(r, c)\}$. Then we say that $f$ is (explicitly) definable in the interval $I$.

If one would like to discuss an arbitrary function, he should introduce a new free variable $\alpha$ of type $[0,1]$, define $f(c)=\{r \mid \alpha[r, c]\}$, and discuss under the hypothesis $\forall \beta(R(\beta) \supset R(\{r \mid \alpha[r, \beta]\}))$.

The number of arguments can be easily increased. The interval $I$ can be the set of all reals, which is certainly definable.

Let $a$ and $b$ be two reals. Then $[a, b],(a, b],[a, b),(a, b)$ are definable in $a$ and $b$. Therefore we can always use general notions of closed or open interval. Let ${ }^{*} A=\{s \mid \exists r>s A(r)\}$. Then ${ }^{*} A$ is arithmetical in $A$. The ${ }^{*}$-operation is very convenient in defining a real from a given set of rationals.

Proposition 3.4.2. Reals are closed under the following operations: $-A,|A|, A+$ $B, A-B, A \cdot B, 1 / B$ and $A / B$, where in the last two $B \neq 0$.

This proposition means that, for example, if $A$ is explicitly definable and $R(A)$, then $-A$ is explicitly definable and $R(-A)$ is provable in FA. The sum $A+B$ appeared in $§ 3.3$.

Proof.

$$
-A=\{t \mid \exists r \exists s(t=-r \wedge s<r \wedge \neg A(s))\} .
$$

$|A|=A \cup(-A)$.

$$
\begin{aligned}
A \cdot B= & *\{t \mid \exists r \exists s(t=r \cdot s \wedge 0<r<A \wedge 0<s<B)\} \\
& \cup^{*}\{t \mid \exists r \exists s(t=r \cdot s \wedge 0<r<-A \wedge 0<s<-B)\} \\
& \cup-^{*}\{t \mid \exists r \exists s(t=r \cdot s \wedge 0<r<-A \wedge 0<s<B)\} \\
& \cup-^{*}\{t \mid \exists r \exists s(t=r \cdot s \wedge 0<r<A \wedge 0<s<-B)\} \\
& \cup\{r \mid r<0 \wedge(A=0 \vee B=0)\} .
\end{aligned}
$$


$A-B=A+(-B)$.

$$
\begin{aligned}
1 / B= & *\{t \mid \exists s(s>0 \wedge \neg B(s) \wedge t=1 / s)\} \\
& \cup\{t \mid \exists r \exists s(s<r<0 \wedge \neg B(s) \wedge t=1 / r)\} .
\end{aligned}
$$

$A / B=A \cdot 1 / B$.

In order to show that these are reals, recall the lemma immediately above.

Proposition 3.4.3. The functions $-,||,+, \cdot, \div$ are explicitly definable functions, defined for all reals (except $B=\hat{0}$ for the last case).

It is only a matter of routine work to establish all the basic properties about those functions. Try $(x+y) z=x z+y z$.

Proposition 3.4.4. The composition of functions is explicitly definable.

Proof. As an example, consider the following case. Let $A(a), B(a)$ and $C(b, c)$ be abstracts which define functions $f(a), g(a)$ and $h(b, c)$. Then $R(A(a))$ and $R(B(a))$, if $a$ belongs to an appropriate interval, and $C(A(a), B(a))$, which is again an abstract, defines $h(f(a), g(a))$. We are of course assuming that the values of $f(a)$ and $g(a)$ belong to the domain of $h$.

Definition 3.4.2. Let $f$ be a function (on a domain $D$ ).

1) $f$ is said to be continuous at a point $c$ in $D$ if

$$
\forall \varepsilon>0 \exists \delta>0 \forall x \in D(|x-c|<\delta \supset|f(x)-f(c)|<\varepsilon)
$$

where $\varepsilon$ and $\delta$ range over rationals, while $x$ stands for reals. Here $|x-c|<\delta$ is, more precisely, $|x-c|<\hat{\delta}$.

2) $f$ is continuous on $D$ if

$$
\forall c \in D \forall \varepsilon>0 \exists \delta>0 \forall x \in D(|x-c|<\delta \supset|f(x)-f(c)|<\varepsilon)
$$

3) $f$ is uniformly continuous on $D$ if

$$
\forall \varepsilon>0 \exists \delta>0 \forall x, y \in D(|x-y|<\delta \supset|f(x)-f(y)|<\varepsilon) .
$$

4) $f$ is bounded above if $\exists r \forall x \in D(f(x)<r)$; similarly with a lower bound.

We can generalize the notions defined above to functions of several arguments in an obvious manner.

Note that $\varepsilon$ and $\delta$ (the familiar letters in calculus!) stand for rationals. In calculus whether you take these as any reals or restrict them to rationals it comes out the same. The following propositions are easily proved in FA.

Proposition 3.4.5. The composition of continuous functions is continuous.

Proposition 3.4.6. All the familiar functions are definable and continuous; ,,$+- \times, \div$ (if the second argument is not 0 ), || , etc. 


\subsection{Sequences}

Definition 3.5.1. Let $A:\{r\} A(s, r)$ be an abstract of type 1 . Then $A$ is called a sequence of reals if $\forall s(N(s) \supset R(\{r\} A(s, r)))$. Or we do the discussion on sequences under the assumption that $\forall s(N(s) \supset R(\{r\} A(s, r)))$.

We will let $n, m, \ldots$ stand for natural numbers. So an expression such as $\forall s(N(s) \supset R(\{r\} A(s, r)))$ can be written as $\forall n R(\{r\} A(n, r))$. The fact that $A$ is a sequence may be symbolically expressed as $\{r\} A(n, r)$ (regarding $n$ as varying) or $\left\{A_{n}\right\}$ or even $\left\{a_{n}\right\}$.

Definition 3.5.2. A sequence $\left\{a_{n}\right\}$ is bounded above if $\exists r \forall n\left(a_{n}<r\right)$ (r rational); bounded below if $\exists r \forall n\left(r<a_{n}\right)$; and bounded if $\exists r \forall n\left(\left|a_{n}\right|<r\right)$.

Proposition 3.5.1. The supremum of a sequence, which is bounded above exists: namely, the sup of a sequence which is bounded from above is real. A proposition about inf can be stated in a symmetric manner.

Proof. Let $A:\{r\} A(n, r)$ be such a sequence with an upper bound. Then the sup of $A$, which we denote $\sup _{n} A$ or $\sup _{n} a_{n}$ is defined to be $\{r\}(\exists n A(n, r))$, which is an abstract. We will show that

1) $\sup a_{n}$ is a real.

2) $a_{n} \leq \sup a_{n}$.

3) If $a_{n} \leq a$ for every $n$, then $\sup _{n} a_{n} \leq a$.

Since each $a_{n}$ is a Dedekind cut of rationals, and $\left\{a_{n}\right\}$ is bounded from above, $\sup _{n} a_{n}=\bigcup_{n} a_{n}=\bigcup_{n}\{r \mid A(n, r)\}$ defines a Dedekind cut. 2) and 3) are also obvious.

We define the inf by the following equation

$$
\inf _{n} a_{n}=^{*}\{r \mid \forall n A(n, r)\}
$$

provided that $\left\{a_{n}\right\}$ is $\{r \mid A(n, r)\}$ and $\left\{a_{n}\right\}$ has a lower bound.

As we have just done, we will often employ set theoretical arguments; they can be easily rewritten in our system.

Proposition 3.5.2. Let $\left\{a_{n}\right\}$ be a sequence. Then limsup, liminf and lim of $\left\{a_{n}\right\}$ are definable abstracts; namely there are abstracts of type 1 which express those notions (and the necessary properties are provable in FA).

Proof. Let $\{r\} A(n, r)$ be the abstract which defines $\left\{a_{n}\right\}$. Then $\lim \sup a_{n}$ is defined as $*\{r\}(\forall n \exists m \geq n A(m, r)\}$, which is an abstract. Furthermore $\lim \inf a_{n}$ is defined to be ${ }^{*}\{r\}(\exists n \forall m \geq n A(m, r))$. In order to see that those definitions are appropriate, show that $\forall \varepsilon>0 \exists m\left(\left|\left(\lim \sup a_{n}\right)-a_{m}\right|<\varepsilon\right)$ and 
$\forall \varepsilon>0 \exists m\left(\left|a-a_{m}\right|<\varepsilon\right) \rightarrow a \leq \limsup a_{n}$ are provable in FA, presuming that $\lim \sup a_{n}$ is a real (namely not $\left.\infty\right)$. We define $\lim a_{n}$ to be $\lim \sup a_{n}$ (or $\left.\liminf a_{n}\right)$ when $\limsup a_{n}=\liminf a_{n}$. Thus, when we make a statement about $\lim a_{n}$, it is a statement about $\lim \sup a_{n}$, under the assumption that it is equal to $\lim \inf a_{n}$. Consequently, a formula such as $a=\lim a_{n}$ is an abbreviation of

$$
\limsup a_{n}=\liminf a_{n} \wedge a=\lim \sup a_{n} .
$$

We can show that

$$
a=\lim a_{n} \leftrightarrow \forall \varepsilon>0 \exists N \forall n \geq N\left(\left|a-a_{n}\right|<\varepsilon\right)
$$

is provable in FA. If one of those conditions (hence both) holds, then we say that $a$ is the limit of $\left\{a_{n}\right\}$ or that $\left\{a_{n}\right\}$ converges to $a$. So we can also say that the limit of a sequence is definable as an abstract.

Proposition 3.5.3. $\lim a_{n}=a \wedge \lim b_{n}=b \rightarrow \lim \left(a_{n}+b_{n}\right)=a+b$ and $\lim a_{n}=a \wedge \lim b_{n}=b \rightarrow \lim \left(a_{n}-b_{n}\right)=a-b$ are provable in $F A$.

Definition 3.5.3. A sequence $\left\{a_{n}\right\}$ is said to be a Cauchy sequence if $\forall \varepsilon>$ $0 \exists N \forall n, m \geq N\left(\left|a_{n}-a_{m}\right|<\varepsilon\right)$.

Proposition 3.5.4. A sequence $\left\{a_{n}\right\}$ is a Cauchy sequence if and only if it is convergent, where by " $\left\{a_{n}\right\}$ is convergent" we mean $\lim a_{n}$ exists (and is a real).

Proof. The "If" part is proved as usual. The "Only if" part, which is usually a consequence of the completeness of the reals, is established by recalling the definition of reals (Dedekind cuts of rationals). Suppose $\left\{a_{n}\right\}$ is Cauchy. We can show that a Cauchy sequence is bounded. Let $\varepsilon=1$ and let $N$ be the corresponding natural number. Then $\max \left(a_{N+1}+1, a_{1}, \ldots, a_{N}\right)$ can be an upper bound. The maximum can be defined as an abstract as follows.

$$
\left\{r \mid r<a_{N+1}+1 \vee \exists n \leq N\left(r<a_{n}\right)\right\}
$$

Similarly with a lower bound. Therefore $\lim \sup a_{n}$ and $\liminf a_{n}$ exist. We know that $\lim \inf a_{n} \leq \lim \sup a_{n}$. So if we can show the opposite inequality, we are done. Note that $\liminf a_{n}=^{*} \bigcup \bigcap_{m>m} a_{n}$ and $\lim \sup a_{n}=^{*} \bigcap_{m} \bigcup_{n>m} a_{n}$.

If liminf $<\limsup$, then there exist rationals $r_{1}, r_{2}$ such that $r_{1}<r_{2}, \exists s>r_{2}$ ( $s \in \limsup$ ), and $\exists s<r_{1}(s \notin \liminf )$.

These imply

1) $r_{1}<r_{2}$

2) $\forall m \exists n \geq m\left(r_{2} \in a_{n}\right)$

3) $\forall m \exists n^{\prime} \geq m\left(r_{1} \notin a_{n^{\prime}}\right)$. 
Since $n$ and $n^{\prime}$ depend on $m$, let us write them as $n(m)$ and $n^{\prime}(m)$ respectively. We may assume $\{n(m)\}$ and $\left\{n^{\prime}(m)\right\}$ are increasing in $m$.

Now, for every $\varepsilon>0$, there exists an $N_{\varepsilon}$ such that, if $n, n^{\prime} \geq N_{\varepsilon}$, then

$$
\left|a_{n}-a_{n^{\prime}}\right|<\varepsilon .
$$

Take $\varepsilon>0$ such that $\varepsilon<r_{2}-r_{1}$. Then we have a contradiction. Note that all notions involved here are arithmetical and that the whole argument can be carried out in FA.

\subsection{Continuous functions}

Theorem 3.6.1. Let $f$ be a continuous function defined on $[a, b]$. Then (1) $f$ is uniformly continuous, (2) $f$ is bounded above and below, and (3) $f$ attains its maximum and minimum values in $[a, b]$.

Proof. We continue to observe our notational convection for real and rational numbers. We also use $r$ in the place of $\hat{r}$.

(1) Given $\varepsilon>0$, we wish to show a contradiction from the hypothesis

$$
\forall \delta>0 \exists x, y \in[a, b](|x-y|<\delta \wedge|f(x)-f(y)| \geq \varepsilon) .
$$

Fix an arithmetical enumeration of rational numbers in $[a, b]$, say $\left(s_{0}, t_{0}\right),\left(s_{1}, t_{1}\right),\left(s_{2}, t_{2}\right), \ldots$. (This is easily done since there are arithmetical one-to-one correspondences between $\mathbb{N}$ and $\mathbb{N} \times \mathbb{N}$ and between $\mathbb{N}$ and $\mathbb{Q}$, where $\mathbb{N}$ is the set of all natural numbers and $\mathbb{Q}$ is the set of all rational numbers.)

Using the hypothesis, we can arithmetically define $\left(s_{n}^{\prime}, t_{n}^{\prime}\right)$ to be the first pair in the enumeration satisfying.

$1^{\circ}$

$$
\left|s_{n}^{\prime}-t_{n}^{\prime}\right|<\frac{1}{n}
$$

$2^{\circ}$

$$
\left|f\left(s_{n}^{\prime}\right)-f\left(t_{n}^{\prime}\right)\right| \geq \varepsilon .
$$

Define $c=\lim _{n} \sup s_{n}^{\prime}$. By Proposition 3.5.2, $c$ is a real and $c \in[a, b]$. Now the contradiction easily follows from the continuity of $f$ at $c$.

Now I would like to remark that the whole proof can be carried out in FA. First, $c$ is defined as an abstract in FA, since only arithmetical notion in involved there. Next, we used that $f$ is continuous at $c$. In the form of inference, this becomes $\forall$ left whose abstract is $c$.

It should be remarked that $\delta$ satisfying

$$
\forall x, y \in[a, b](|x-y|<\delta \supset|f(x)-f(y)|<\varepsilon)
$$

is arithmetically definable i.e. define $\delta$ to be $\frac{1}{n}$ with the condition

$$
\forall s_{1}, s_{2} \in[a, b]\left(\left|s_{1}-s_{2}\right|<\frac{2}{n} \supset\left|f\left(s_{1}\right)-f\left(s_{2}\right)\right|<\frac{\varepsilon}{2}\right) .
$$


(2) We want to define upper and lower bounds for $f$. Use the uniform continuity of $f$ (which we just proved) with $\varepsilon=1$. Then there is a definable positive rational $\delta$ such that if $x, y \in[a, b]$ and $|x-y|<\delta$, then $|f(x)-f(y)|<1$. Let $N$ be the largest integer such that $a+N \delta \leq b$. Then $1+\max (f(a), f(a+$ $\delta), \ldots, f(a+N \delta))$ and $-1+\min (f(a), f(a+\delta), \ldots, f(a+N \delta))$ are definable upper and lower bounds for $f$, respectively. Here max and min can be defined as an abstract.

(3) Let $d=\{s \mid \exists r(a \leq r<b \wedge s<f(r))\}$. From (2) it follows that $d$ is a real number. We claim that $d$ is an upper bound of $f$ in $[a, b]$. Suppose otherwise that is, suppose there were an $x \in[a, b]$ such that $d<f(x)$. Then there exists an $\varepsilon>0$ such that $0<\varepsilon<f(x)-d$. Since $f$ is continuous at $x$, there exists a rational number $r \in[a, b]$ such that

$$
d<f(x)-\varepsilon<f(r)
$$

which is a contradiction.

Suppose now $f(a)<d$.

Now we would like to show that

$$
\exists x \in[a, b] \quad f(x)=d .
$$

For every positive natural number $n$, define $p_{n}$ by the following equation.

$$
p_{n}=^{*}\left\{r \mid a \leq r<b \wedge \forall s\left(a \leq s \leq r \supset f(s)<d-\frac{1}{n}\right)\right\} .
$$

Then $a \leq p_{n} \leq b$. If $p=\sup p_{n}$, it is immediate that $d=f(p)$. It is also clear that the arguments in (2) and (3) can be carried out in FA.

Theorem 3.6.2 (Intermediate Value Theorem).

Suppose $f$ is continuous in $[a, b], f(a)<0$, and $f(b)>0$. Then there exists a definable real $d$ in $(a, b)$ such that $f(d)=0$.

Proof. If

$$
d={ }^{*}\{r \mid a \leq r \leq b \wedge \forall s(a \leq s \leq r \supset f(s)<0)\},
$$

then

1) $d$ is a real number and $a<d<b$.

This is clear since $f$ is continuous at $a$ and $b$.

2) $0 \leq f(d)$.

Suppose $f(d)<0$. Since $f$ is continuous at $d$,

$$
\exists \delta>0 \forall r(|r-d|<\delta \supset f(r)<0)
$$

which contradicts the definition of $d$. 
3) $f(d) \leq 0$.

Suppose $0<f(d)$. Since $f$ is continuous at $d$,

$$
\exists \delta>0 \forall r(|r-d|<\delta \supset f(r)>0) .
$$

But this contradicts the definition of $d$.

Theorem 3.6.3 (Inverse function theorem).

Let $f$ be a continuous monotone function defined in $[a, b]$. Then there is an explicitly definable function $h$ (called the inverse function of $f$ ) from $[f(a), f(b)]$ onto $[a, b]$ such that $h(f(x))=x$ for every $x$ in $[a, b]$.

Proof. Let us assume $f$ is increasing. For each $x$ in $[f(a), f(b)]$ define $h(x)={ }^{*}$ $\{r \mid r \in[a, b) \wedge \forall s(s \in[a, r] \supset f(s)<x)\}$. Then $h$ is an explicitly definable function. The proof that $h(f(x))=x$ is straightforward.

\subsection{Differentiation}

Proposition 3.7.1 (Limits of functions).

Let $f$ be a function (on some domain) which is continuous around a. (That is, for any x "close to" $a, f$ is continuous at $x$. But, $f$ is not necessarily continuous or even defined at a). Then

$$
\limsup _{x \rightarrow a} f(x), \limsup _{x \rightarrow a+0} f(x), \limsup _{x \rightarrow a-0} f(x)
$$

are definable; hence, if not $\infty$, they are definable reals. The same is true with $\liminf$ of $f$.

Proof. We do one case as an example. The usual definition of $\limsup _{x \rightarrow a+0} f(x)$ is

$$
{ }_{\varepsilon>x>0}^{*} \bigcup_{0<y<x} f(a+y)
$$

Since $f$ is continuous around $a$, this is the same as

$$
\bigcap_{\varepsilon>r>0}^{*} \bigcup_{0<s<r} f(a+s)
$$

which is an abstract in FA.

Definition 3.7.1. If $\limsup _{x \rightarrow a} f(x)=\liminf _{x \rightarrow a} f(x)$, then we call either of them $\lim _{x \rightarrow a} f(x)$.

Proposition 3.7.2. Let $f$ be continuous around a. 
1) If $\limsup _{x \rightarrow a} f(x)$ exists and $\limsup _{x \rightarrow a} f(x)<b$,

$$
\begin{array}{lc}
\begin{array}{c}
x \rightarrow a \\
\text { then }
\end{array} & \exists r<a \\
& \exists r<b \exists>0 \forall x(0 \neq|x-a|<\varepsilon \supset f(x)<r) .
\end{array}
$$

2) If $\liminf _{\substack{x \rightarrow a \\ \text { then }}} f(x)$ exists and $\liminf _{x \rightarrow a} f(x)>b$,

$$
\exists r>b \exists \varepsilon>0 \forall x(0 \neq|x-a|<\varepsilon \supset r<f(x)) .
$$

A similar statement also holds for $\limsup _{x \rightarrow a+0}$

$$
\limsup _{x \rightarrow a-0}, \liminf _{x \rightarrow a+0} \text {, or } \liminf _{x \rightarrow a-0} .
$$

Proof. We prove only 1). Under the hypothesis of 1 ), we would like to show that

$$
\exists r<b \exists \varepsilon>0 \forall x(0 \neq|x-a|<\varepsilon \supset f(x)<r) .
$$

It suffices to show that

$$
\exists r<b \exists \varepsilon>0 \forall x(0 \neq|x-a|<\varepsilon \supset f(x) \leq r) .
$$

Now $\limsup _{x \rightarrow a} f(x)<b$ implies that

$$
\bigcap_{\varepsilon>r>0} \bigcup_{0<\varepsilon<r} f(a+s)<b
$$

From this it follows that

$$
\exists r<b \exists n>0 \forall s\left(0 \neq|s|<\frac{1}{n} \supset f(a+s) \leq r\right) .
$$

Since $f$ is continuous around $a$, the proposition is proved.

Proposition 3.7.3. If $f$ is continuous around $a$ and $\lim _{x \rightarrow a} f(x)$ exists, then the following equivalences hold.

1) $c=\lim _{x \rightarrow a} f(x)$ iff

$$
\forall \varepsilon>0 \exists \delta>0 \forall x(0 \neq|x-a|<\delta \supset|f(x)-c|<\varepsilon) .
$$

2) $f$ is continuous at a iff $\lim _{x \rightarrow a} f(x)=f(a)$.

A similar statement to 1) also holds for $\lim _{x \rightarrow a+0}$ or $\lim _{x \rightarrow a-0}$.

Proof. 1) Follows easily from Proposition 3.7.2.

2 ) Follows from 1) immediately.

Other basic properties of lim can be proved. 
Proposition 3.7.4. 1) $\lim _{x \rightarrow a}(f(x)+g(x))=\lim _{x \rightarrow a} f(x)+\lim _{x \rightarrow a} g(x)$

if

$$
\lim _{x \rightarrow a} f(x) \text { and } \lim _{x \rightarrow a} g(x) \text { exist. }
$$

2) Suppose $f$ and $g$ are continuous in appropriate domains, $\lim _{x \rightarrow a} f(x)(=b)$ exists and $\lim _{y \rightarrow b} g(y)$ exists. Then $\lim _{x \rightarrow a} g(f(x))$ exists.

Definition 3.7.2. Assuming that $\lim _{x \rightarrow 0} \frac{f(a+x)-f(a)}{x}$ exists.

$$
f^{\prime}(a)=\lim _{x \rightarrow 0} \frac{f(a+x)-f(a)}{x}
$$

We say that $f$ is differentiable at a if $f$ is continuous at a and $f^{\prime}(a)$ exists. If $f$ is differentiable at every point in the domain of $f$, then we say $f$ is differentiable.

The following propositions are proved by the usual methods.

Proposition 3.7.5. The composition of differentiable functions are differentiable.

Theorem 3.7.6 (Rolle's Theorem). Suppose $f$ is differentiable in $[a, b]$ and $f(a)=f(b)$. Then there exists a definable real $c$ in $(a, b)$ such that $f^{\prime}(c)=0$.

Theorem 3.7.7 (Mean Value Theorem). Suppose $f$ is differentiable in $[a, b]$. Then there exists a definable real $c$ in $(a, b)$ such that

$$
\frac{f(b)-f(a)}{b-a}=f^{\prime}(c)
$$

\subsection{Integration}

We will define the integral as the limit of a finite sum.

Proposition 3.8.1. Let $\left\{a_{n}\right\}$ be a sequence of reals. Then

$$
\left\{\sum_{i=1}^{n} a_{i}\right\}
$$

is also a sequence of reals.

Proof. Since a rational can be considered as an ordered triple of natural numbers and ordered $n$-tuples of natural numbers can be arithmetically made to correspondence one-to-one with natural numbers, ordered $n$-tuples of rational numbers $(n=0,1,2, \ldots)$ can be arithmetically made to one-to-one correspondence with rational numbers. The arithmetical formula " $s$ is an ordered $n$-tuple 
of rational numbers" is denoted by $\operatorname{Ord}(n, s)$. The $i$-th term of $s$ is denoted by $(s)_{i}$. Then

$$
\sum_{i=1}^{n}(s)_{i}
$$

can be arithmetically definable and

$$
\sum_{i=1}^{n} a_{i}=\left\{t \mid \exists s\left(\operatorname{Ord}(n, s) \wedge \forall i\left(1 \leq r \leq n \supset(s)_{i} \in a_{i}\right) \wedge t=\sum_{i=1}^{n}(s)_{i}\right)\right\} .
$$

Thus

$$
\left\{\sum_{i=1}^{n} a_{i}\right\}
$$

is a sequence of reals.

Definition 3.8.1. Consider an abstract $\{n, i\} A(n, i)$. Suppose that, for every $n, A_{n}=\{i\} A(n, i)=\left\{a_{i}^{n}\right\}_{i}$ is a sequence of reals for which $\forall i \geq \pi(n)\left(a_{i}^{n}=0\right)$, where $\pi$ is a function from natural numbers to natural numbers which is strictly increasing. We say that $A:\{n, i\} A(n, i)$ is a partition of $[a, b]$ if the following condition (denoted by $P(a, b, A))$ holds:

$$
\begin{gathered}
\forall n\left[\forall i\left(0<i \leq \pi(n) \supset a_{i-1}^{n}<a_{i}^{n} \wedge a_{0}^{n}=a \wedge a_{\pi(n)}^{n}=b\right)\right] \\
\wedge\left[\forall \varepsilon>0 \exists N \forall n \geq N\left(\max _{1 \leq i \leq \pi(n)}\left|a_{i}-a_{i-1}\right|<\varepsilon\right)\right] .
\end{gathered}
$$

The latter condition can be written as $\lim _{n \rightarrow \infty} \max _{1 \leq i \leq \pi(n)}\left|a_{i}-a_{i-1}\right|=0$.

An example would be $a_{i}^{n}=a+\frac{(b-a)}{n} i$.

Proposition 3.8.2. Let $\left\langle\left\{A_{n}\right\}_{n}, \pi\right\rangle$ be a partition of $[a, b]$ and let $f$ be a continuous function defined on $[a, b]$, where $A_{n}=\left\{a_{i}^{n}\right\}_{i}$. Then

$$
\lim _{n \rightarrow \infty} \sum_{i=1}^{\pi(n)} f\left(a_{i}^{n}\right)\left(a_{i}^{n}-a_{i-1}^{n}\right)
$$

exists and the limit does not depend on the partition, that is, the limit is uniquely determined by $f$ and $[a, b]$.

Proof. Follow the ordinary proof. What we need is the uniform continuity of $f$ on $[a, b]$ which we proved in Theorem 3.6.1.

Definition 3.8.2. Let $f$ be a continuous function on $[a, b]$. We define $\int_{a}^{b} f(x) d x$ by

$$
\int_{a}^{b} f(x) d x=\lim _{n \rightarrow \infty} \sum_{i=1}^{n} f\left(a_{i}^{n}\right)\left(a_{i}^{n}-a_{i-1}^{n}\right)
$$

where $a_{i}^{n}=a+\frac{(b-a)}{n} \cdot i$. 
By Proposition 3.8.2, $\int_{a}^{b} f(x) d x$ is a definable abstract and is shown to be a real number.

Various, basic properties of integrals can be easily shown in our system. We assume all functions are continuous in $[a, b]$.

Proposition 3.8.3. 1) If $f(x) \leq g(x)$ in $[a, b]$, then

$$
\int_{a}^{b} f(x) d x \leq \int_{a}^{b} g(x) d x
$$

2) $\int_{a}^{b} f(x) d x+\int_{b}^{c} f(x) d x=\int_{a}^{c} f(x) d x$

Proposition 3.8.4. Suppose $f$ is continuous in $[a, b]$ and $x$ stands for any number in $[a, b]$. Then $\int_{a}^{x} f(x) d x$ can be defined as a function of $x$ in $[a, b]$.

Proposition 3.8.5. If $F(x)=\int_{a}^{x} f(x) d x$, then $F$ is continuous in $x$; in fact $F$ is differentiable and $F^{\prime}(x)=f(x)$.

\subsection{Sequences of functions}

Definition 3.9.1. 1) An arithmetical abstract of type $[0,1,0]$ say $\{n, x, r\} A(n, x, r)$, is called a sequence of functions (defined on a domain $D$ ) if $\forall n \forall x \in$ $D R(\{r\} A(n, x, r))$. We denote such functions by $f_{n}(x)=\{r \mid A(n, x, r)\}$.

2) Suppose $F=\left\{f_{n}\right\}$ is a sequence of functions (on $D$ ). We say that $F$ is pointwise convergent on $D$ if $\forall x\left(\lim \sup f_{n}(x)=\liminf f_{n}(x) \wedge R\left(\limsup f_{n}(x)\right)\right)$, where the last condition means that the value is not $\infty$. If $F$ is convergent, then we denote either value of the above equation by $\lim _{n} f_{n}(x)$. (If we say $F$ is convergent we mean $F$ is pointwise convergent.) ${ }^{n}$

It is obvious that $\lim _{n} f_{n}(x)$ as a function of $x$ is definable. Since we consider pointwise convergent, all the properties on the limit of $f_{n}$ follows from the properties of the limit of reals by the routine method. Therefore we omit the proof in the following.

Proposition 3.9.1. $f(x)=\lim _{n} f_{n}(x)$ for every $x$ in $D$ if and only if $\forall x \in$ $D \forall \varepsilon>0 \exists N \forall n\left(n \geq N \supset\left|f(x)-f_{n}(x)\right|<\varepsilon\right)$.

If one (hence both) of those holds, we say that $F=\left\{f_{n}\right\}$ converges to $f$ (pointwise on D) and write $f=\lim _{n} f_{n}$.

The sequence of functions $F=\left\{f_{n}\right\}$ is said to be uniformly convergent on $D$ if $F$ is convergent (pointwise) and

$$
\forall \varepsilon>0 \exists N \forall x \in D \forall n\left(n \geq N \supset\left|\left(\lim _{m} f_{m}(x)\right)-f_{n}(x)\right|<\varepsilon\right) .
$$


Proposition 3.9.2. $f=\lim f_{n}$ and $\lim f_{n}$ is uniformly convergent if and only if $\forall \varepsilon>0 \exists N \forall x \in D \forall n\left(n \geq N \supset\left|f(x)-f_{n}(x)\right|<\varepsilon\right)$.

If this is the case, we say that $F=\left\{f_{n}\right\}$ converges to $f$ uniformly on $D$.

Definition 3.9.2. Let $F=\left\{f_{n}\right\}$ be a definable sequence of functions. If $f_{n}$ is continuous for every $n$, then we say that $F$ is continuous.

Definition 3.9.3. The sequence of functions $F=\left\{f_{n}\right\}$ has the Cauchy property (is Cauchy) (uniformly on D) if

$$
\forall \varepsilon>0 \exists N \forall x \in D \forall n, m\left(n, m \geq N \supset\left|f_{n}(x)-f_{m}(x)\right|<\varepsilon\right) .
$$

Theorem 3.9.3. The sequence of functions $F=\left\{f_{n}\right\}$ is Cauchy (on D) if and only if $F$ is uniformly convergent on $D$.

Theorem 3.9.4. If $F$ is continuous and converges uniformly to $f$, then $f$ is continuous.

Theorem 3.9.5. Suppose $F=\left\{f_{n}\right\}$ is continuous and converges to $f$ uniformly in an interval 1 . Suppose also that $\int_{I} f_{n}$ exists ( $f_{n}$ is integrable) for every $n$. Then $\left\{\int_{I} f_{n}\right\}_{n}$ is a definable sequence of numbers and $\lim _{n} \int_{I} f_{n}=\int_{I} f$.

\subsection{Infinite series and series of functions}

The content of this section is also done by routine methods. We simply list definitions and theorems without proof. By looking at them, one can easily see that everything can be done in FA.

Definition 3.10.1. Let $\left\{a_{n}\right\}_{n}$ be a sequence of numbers. Then

$$
\sum_{n=1}^{\infty} a_{n}
$$

is defined to be

$$
\underset{n}{\limsup } \sum_{i=1}^{n} a_{i} .
$$

Such an object is called an infinite series (of $\left\{a_{n}\right\}_{n}$ ). If this is equal to

$$
\liminf _{n} \sum_{i=1}^{n} a_{i}
$$

and not $\infty$, then we say that the series converges and the value is

$$
\lim _{n} \sum_{i=1}^{n} a_{i},
$$

which is a real number. 
All the basic properties about infinite series follow.

Definition 3.10.2. Let $F=\left\{f_{n}\right\}$ be a sequence of functions (defined on a do main D). Then

$$
\sum_{n=1}^{\infty} f_{n}
$$

is defined to be the abstract $\{x\}$ limsup

$$
\limsup _{n} \sum_{i=1}^{n} f_{i}(x)
$$

(We could start the sum with $n=0$.) This is called the series of F. If there is a subdomain of $D$, say $E$, on which

$$
\underset{n}{\limsup } \sum_{i=1}^{n} f_{i}(x)=\liminf _{n} \sum_{i=1}^{n} f_{i}(x)(=\text { finite }),
$$

then we say that the series

$$
\sum_{n=1}^{\infty} f_{n}
$$

converges in $E$ pointwise (and the value for each $x$ is

$$
\left.\lim _{n} \sum_{i=1}^{n} f_{i}(x)\right)
$$

Definition 3.10.3. A series of functions, say

$$
\sum_{n=1}^{\infty} f_{n}
$$

is said to converge on $D$ uniformly if it converges pointwise and the convergence is uniform.

Theorem 3.10.1. If

$$
\forall \varepsilon>0 \exists N \forall n, m \geq N \forall x\left(\left|\sum_{i=n}^{m} f_{i}(x)\right|<\varepsilon\right),
$$

then $\sum f_{n}$ is uniformly convergent.

Theorem 3.10.2 (Weinstrass Comparison Test).

Let $F=\left\{f_{n}\right\}$ be a definable sequence of functions and let $\left\{M_{n}\right\}$ be a definable sequence of positive numbers. If $\forall x\left(\left|f_{n}(x)\right| \leq M_{n}\right)$ for every $n$, and $\sum M_{n}$ converges, then $\sum f_{n}$ is uniform convergent. 
Theorem 3.10.3. Suppose $F=\left\{f_{n}\right\}$ is definable and continuous and $\sum f_{n}$ converges to $f$ uniformly, then $f$ is continuous.

Theorem 3.10.4. If $F=\left\{f_{n}\right\}$ is continuous and $\sum f_{n}$ converges to $f$ uniformly on $I$, where $I$ is our interval, and each $f_{n}$ is integrable over $I$, then $\int_{I} f=\sum \int_{I} f_{n}$

Theorem 3.10.5. Suppose that $F=\left\{f_{n}\right\}$ is continuous in $I=[a, b]$, that $f_{n}^{\prime}$ exists for each $n$, that $F^{\prime}=\left\{f_{n}^{\prime}\right\}$ is continuous, and $\sum f_{n}$ converges to $f$ in $I$ (uniformly). If $\sum f_{n}^{\prime}$ converges uniformly in $I$, then $\sum f_{n}^{\prime}=f^{\prime}$.

Proposition 3.10.6. Let $\left\{a_{n}\right\}$ be a sequence. Then $\prod_{i=1}^{n} a_{i}$ is defined as an abstract.

Proof. The properties 'there is a zero among $a_{i}(1 \leq i \leq n)$ ' and 'there are an even number of negative numbers among $a_{i}(1 \leq i \leq n)^{\prime}$ ' are arithmetical. So the problem is reduced to the case that all $a_{i}(1 \leq i \leq n)$ are positive. In this case it is expressed by

$$
*\left\{t \mid \exists s\left(\operatorname{Ord}(n, s) \wedge \forall i\left(1 \leq i \leq n \supset 0<(s)_{i} \in a_{i}\right) \wedge t=\prod_{i=1}^{n}(s)_{i}\right)\right\} .
$$

Proposition 3.10.7. The power $a^{n}$ is defined as an abstract.

Proposition 3.10.8. Let $\left\{a_{i}\right\}_{i}$ be a sequence of numbers. Then $F=\left\{a_{i} x^{i}\right\}$ $(i=0,1,2, \cdots)$ is a sequence of functions. (In fact $F$ is continuous.) Therefore

$$
\sum_{i=0}^{\infty} a_{i} x^{i}\left(=\underset{n}{\limsup } \sum_{i=0}^{n} a_{i} x^{i}\right)
$$

is definable (as a function of $x$ ).

We call a series of function, in the above form, a power series (in $x$ ).

Proposition 3.10.9. The root function $a^{1 / n}$ is a definable function (for positive a) of $a$ and $n$.

Proof. $a^{1 / n}={ }^{*}\left\{r \mid r>0 \wedge(r)^{n}<a\right\}$. (Note that we are taking a positive root.)

Proposition 3.10.10. Let $\sum_{i=0}^{\infty} a_{i} x^{i}$ be a power series in $x$. Then the radius of convergence, say $R$, is definable. 
Proof.

$$
R=\frac{1}{\limsup _{n}\left|a_{n}\right|^{1 / n}}
$$

Every operation here is arithmetical. If the denominator in the right side is 0 , we say that $R=\infty$.

Theorem 3.10.11. Let $\sum a_{i} x^{i}$ be a power series whose radius of convergence is $R$. Then, for every $b, 0<b<R$, the series converges uniformly on $[-b, b]$.

Theorem 3.10.12. Suppose $\sum a_{n} x^{n}$ converges to $f$ for $|x|<R$. Then $f^{\prime}$ exists and

$$
f^{\prime}(x)=\sum_{n=1}^{\infty} n a_{n} x^{n-1} \quad \text { for }|x|<R .
$$

We can extend the notion of power series to those of the form $\sum a_{n}(x-c)^{n}$ for a constant $c$.

\subsection{Higher derivatives}

Definition 3.11.1. Let $f$ be continuous at $x_{0}$ and let $n>0$. Then $f^{(n)}\left(x_{0}\right)$ is defined as follows. For $h \neq 0$, define $\Delta^{n} y_{0}$ by

$$
\Delta^{n} y_{0}=f\left(x_{0}+n h\right)-\left(\begin{array}{c}
n \\
1
\end{array}\right) f\left(x_{0}+(n-1) h\right)+\cdots+(-1)^{n}\left(\begin{array}{l}
n \\
n
\end{array}\right) f\left(x_{0}\right)
$$

Put $h=\Delta x$ and define $f^{(n)}\left(x_{0}\right)$ by

$$
f^{(n)}\left(x_{0}\right)=\lim _{\Delta x \rightarrow 0} \frac{\Delta^{n} y_{0}}{(\Delta x)^{n}} .
$$

Note that $f^{(n)}\left(x_{0}\right)$ is meaningless unless $f(x), f^{\prime}(x), \ldots, f^{(n-1)}(x)$ exist and are continuous at $x_{0}$. If this is the case and $f^{(n)}\left(x_{0}\right)$ exists, then $f^{(n)}\left(x_{0}\right)$ is called the $n$-th derivative of $f$ at $x_{0}$. Moreover $f^{(n)}\left(x_{0}\right)$ is definable as an abstract with $n$ and $x_{0}$. The properties of $f^{(n)}$ are easily shown in FA. (Cf pp. 91-93 in [21].)

We say that a function $f$ is of class $C^{n}$ (or $f \in C^{n}$ ) on a domain $D$ if it is defined on $D$ and for every $k, 0 \leq k \leq n, f^{(k)}$ exists and is continuous on $D$, where $f^{(0)}$ denote $f$.

A function $f$ is of the class $C^{\infty}$ if $f^{(n)}$ exists and is continuous for every $n$ or $F=\left\{f^{(n)}\right\}$ is a continuous sequence of functions.

Note that polynomials are of class $C^{\infty}$.

Proposition 3.11.1. Let $f$ be of the class $C^{n}$ and let $x_{0}$ be a real in the domain. The Taylor polynomial of degree $n$ for $f$ at $x_{0}$ can be defined as an abstract with a parameter $x$. 
Proof. Such a polynomial (say $P$ ) is defined as $P(x)=a_{n}\left(x-x_{0}\right)^{n}+a_{n-1}(x-$ $\left.x_{0}\right)^{n-1}+\cdots+a_{0}$, where $a_{k}=\frac{f^{(n)}\left(x_{0}\right)}{k !}$. The polynomial $P$ is definable.

Definition 3.11.2. The function $R_{n}(x)=f(x)-P(x)$ is called the remainder of $f$ (with respect to $P$ of $f$ at $x$ ). Note that $R_{n}$ is definable.

Theorem 3.11.2. Suppose $f$ is of the class $C^{n+1}$ in an interval $I$ and $x_{0} \in I$. Then for any $x$ in $I$.

$$
R_{n}(x)=\frac{1}{n !} \int_{x_{0}}^{x} f^{(n+1)}(y)(x-y)^{n} d y
$$

Note that the right hand side is also definable.

Definition 3.11.3. A function $f$ is said to be analytic at $x_{0}$ if there is an open interval around $x_{0}$, say $I$, on which $f$ is $C^{\infty}$ and for every $x$ in $I, \lim _{n \rightarrow \infty} R_{n}(x)=$ 0 .

Example. $\sqrt{x}$ for an $x_{0}>0$.

Theorem 3.11.3. If $f$ is analytic at $x_{0}$, then there is a unique, definable sequence of numbers, $\left\{a_{n}\right\}$, such that $\sum_{x=0}^{\infty} a_{n}\left(x-x_{0}\right)^{n}$ converges to $f$ around $x$, that is, $f(x)=\sum_{x=0}^{\infty} a_{n}\left(x-x_{0}\right)^{n}$.

The right hand side of the above equation is called the Taylor series for $f\left(a+x_{0}\right)$.

Proof. The sequence of numbers $a_{n}=\frac{f^{(n)}\left(x_{0}\right)}{n !}$ is uniformly (in $n$ ) definable.

Theorem 3.11.4. Let $f(x)=\sum_{n} a_{n}(x-c)^{n}$ (i.e. the series converges to $f$ ) around c. Then $f^{(n)}$ exists and $a_{n}=\frac{f^{(n)}(c)}{n !}$.

\subsection{Functions of several variables}

Theorem 3.12.1. Let $f(x, y)$ be definable and continuous for $x \in[a, b]$ and $y \in[c, d]$.

Then

1) $f$ is uniformly continuous in $[a, b] \times[c, d]$,

2) $f$ attain its maximum in $[a, b] \times[c, d]$, the maximum is definable, and there exist definable reals $x_{0} \in[a, b]$ and $y_{0} \in[c, d]$ such that $f\left(x_{0}, y_{0}\right)$ is the maximum. 
Proof. 1) goes in the same way as in the proof of Theorem 3.6.1.

2) can be also proved in the same way but we prove it in a different way here.

If $g(x)=\sup _{y \in[c, d]} f(x, y)$, then $g(x)$ is definable and continuous. Therefore $\sup _{x \in[a, b]} g(x)$ is definable.

There exists a definable $x_{0}$ such that

$$
g\left(x_{0}\right)=\sup _{x \in[a, b]} g(x) .
$$

Also, there exists a definable $y_{0}$ such that

$$
f\left(x_{0}, y_{0}\right)=\sup _{y \in[c, d]} f\left(x_{0}, y\right) .
$$

Then $f(x, y)$ attains its maximum at $\left(x_{0}, y_{0}\right)$.

In the same way as in Theorem 3.12.1, we can prove the following theorem.

Theorem 3.12.2. Let $f\left(x_{1}, \ldots, x_{n}\right)$ be continuous in $x_{1} \in\left[a_{1}, b_{1}\right], \ldots, x_{n} \in$ $\left[a_{n}, b_{n}\right]$. Then

1) $f$ is uniformly continuous in $\left[a_{1}, b_{1}\right] \times \cdots \times\left[a_{n}, b_{n}\right]$,

2) $f$ attains its maximum in $\left[a_{1}, b_{1}\right] \times \cdots \times\left[a_{n}, b_{n}\right]$, the maximum is definable, and there exist definable reals $x_{1} \in\left[a_{1}, b_{1}\right], \ldots, x_{n} \in\left[a_{n}, b_{n}\right]$ such that $f\left(x_{1}, \ldots, x_{n}\right)$ is the maximum. 



\section{Chapter 4}

\section{Complex Analysis}

\subsection{A system of complex numbers}

We will formulate the arithmetic of complex numbers in a system of finite types, which is a conservative extension of Peano Arithmetic. The rational complex numbers are objects of type 0 .

Definition 4.1.1. The system $T$.

Symbols: $N, r, 0,1, i,+, \cdot,-, \div,=,<$.

The logical system: The system of finite types defined in Chapter 3.

Axioms:

(1) The equality axiom Eq.

(2) Axioms of Peano Arithmetic relativized to $N$.

(3) MI, the mathematical induction relativized to $N$.

(4) Axioms on $0,1,+, \cdot,-, \div$, and $<$ relativized to $r$.

(5) $\forall x \exists ! y \exists ! z(r(y) \wedge r(z) \wedge x=y+i z)$ where $x, y, z$ are of type 0 and $\exists$ ! expresses unique existence.

(6) $N(a) \rightarrow r(a)$.

(7) Axioms on $i$.

(8) $\forall x(r(x) \wedge x>0 \supset \exists y \exists z(N(y) \wedge N(z) \wedge x=y / z))$.

Remark.

1. The intended meaning of the symbols should be self-evident: $N(a): a$ is a natural number, $r(a): a$ is a real rational, $a+i b$ : a complex rational with the real part $a$ and the imaginary part $b$. The operators $+, \cdot,-, \div$, and $<$ are defined for real rationals. 
2. (2) includes $N(0)$.

3. A variable of type 0 stands for a complex rational.

4. We will often abbreviate $r(a) \wedge r(b) \wedge a<b$ to $a<b$.

Definition 4.1.2. The predicate $C(\alpha): \alpha$ is a complex number, is defined as follows. Let $\alpha$ be a free variable of type 1 . Then $C(\alpha)$ is the following formula:

$$
\begin{gathered}
\exists x \alpha(x) \wedge \exists x \neg \alpha(x) \wedge \forall x \forall y(r(x) \wedge r(y) \supset \\
(a(x+i y) \equiv \exists u \exists v(r(u) \wedge r(v) \wedge x<u \wedge y<v \wedge \alpha(u+i v))) .
\end{gathered}
$$

Proposition 4.1.1. The predicate " $\alpha$ is real" is arithmetical. The "real part" of $\alpha$ and the "imaginary part" of $\alpha$ are definable as abstracts.

Proof. The predicate $R(\alpha): \alpha$ is real, is defined by

$$
C(\alpha) \wedge \forall x \forall y \forall z(r(x) \wedge r(y) \wedge r(z) \wedge \alpha(x+i y) \supset(\alpha(x+i z) \equiv z<0)) .
$$

Similarly $\operatorname{Re}(\alpha)$ : the real part of $\alpha$, is defined as $\{x \mid r(x) \wedge \exists y(r(y) \wedge \alpha(x+i y))\}$, and $\operatorname{Im}(\alpha)$ : the imaginary part of $\alpha$, is defined as $\{y \mid r(y) \wedge \exists x(r(x) \wedge \alpha(x+i y))\}$.

Note that the real part of $\alpha$ is not "real" in our sense, but

$$
C(\alpha) \rightarrow R(\{x+i y \mid x \in \operatorname{Re}(\alpha) \wedge y<0\}) .
$$

Similarly with the imaginary part.

Proposition 4.1.2. A complex rational, as a complex number, is definable as an abstract.

Proof. Let $a$ and $b$ be real rationals. A complex number corresponding to $a+i b$ is

$$
\{x+i y \mid x<a \wedge y<b\} .
$$

As in Chapter 3, a complex number corresponding to a complex rational $a$ should be denoted by $\hat{a}$. However we also use $a$ in the place of $\hat{a}$.

We can define $\alpha=\beta$ by $\forall x(\alpha(x) \equiv \beta(x))$, which is arithmetical.

Proposition 4.1.3. If $C(\alpha)$, then $\operatorname{Re}(\alpha)$ and $\operatorname{Im}(\alpha)$ are reals in the sense of Chapter 3, where all the quantifiers are relativized by $r$. We denote " $\alpha$ is real", in this sense, by $\tilde{R}(\alpha)$.

Proposition 4.1.4. Suppose $\alpha$ and $\beta$ satisfy the condition of reals in the sense of Chapter 3. Let $\alpha+i \beta=\{x+i y \mid \alpha(x) \wedge \beta(y)\}$. Then $C(\alpha+i \beta) \wedge \operatorname{Re}(\alpha+$ $i \beta)=\alpha \wedge \operatorname{Im}(\alpha+i \beta)=\beta$. With this notation, $C(\alpha) \rightarrow \alpha=\operatorname{Re}(\alpha)+i \operatorname{Im}(\alpha)$. Furthermore, $C(\alpha) \rightarrow \exists ! \varphi \exists ! \psi(\tilde{R}(\varphi) \wedge \tilde{R}(\psi) \wedge \alpha=\varphi+i \psi)$. Then $\alpha+i \beta$ in this context is definable in $\alpha$ and $\beta$. 
Proposition 4.1.5. If $\tilde{R}(\alpha)$ and $\tilde{R}(\beta)$, then the four arithmetic operations and $<$ are defined for $\alpha$ and $\beta$.

Proof. The definitions in Chapter 3 relativized by $r$ will do. For example,

$$
\alpha+\beta=\{z \mid \exists x \exists y(r(x) \wedge r(y) \wedge z=x+y \wedge \alpha(x) \wedge \beta(y))\} .
$$

Remark. Proposition 4.1.5 suggests that we may assume the theory of reals in Chapter 3 for the reals in the sense of $\tilde{R}$.

Proposition 4.1.6. The four arithmetic operations for complex numbers are definable.

Proof. Suppose $C(\alpha) \wedge C(\beta)$. Let $a=\operatorname{Re}(\alpha), b=\operatorname{Im}(\alpha), c=\operatorname{Re}(\beta)$ and $d=\operatorname{Im}(\beta)$.

$$
\begin{aligned}
& \alpha+\beta=(a+c)+i(b+d) ; \\
& \alpha-\beta=(a-c)+i(b-d) ; \\
& \alpha \cdot \beta=(a c-b d)+i(a d+b c) ; \\
& \frac{\alpha}{\beta}=\frac{a c+b d}{c^{2}+d^{2}}+i \frac{-a d+b c}{c^{2}+d^{2}},
\end{aligned}
$$

where the terms in the right hand side of each equation are defined as in Proposition 4.1.5.

Proposition 4.1.7. (1) Let $\alpha=a+i b$ (cf. Proposition 4.1.6). Then $\bar{\alpha}=$ $a+i(-b)$ (the conjugate of $\alpha$ ) (which is denoted by $a-i b)$ is definable (in $\alpha)$.

(2) $|\alpha|=\sqrt{a^{2}+b^{2}}$ (the absolute value of $\alpha$ ) is definable.

Corollary 4.1.8. $\operatorname{Re}(a)=\frac{\alpha+\bar{\alpha}}{2} ; \operatorname{Im}(a)=\frac{\alpha-\bar{\alpha}}{2 i}$ where $i$ is an abbreviation of $0+i \cdot 1$.

In the subsequent discussion, we employ the usual notations: $\alpha=a+i 0$ is denoted by $a ; 0+i b$ is denoted by $i b ; a+i 1$ is denoted by $a+i$. Numerals $1,2, \ldots$ in this context stand for reals rather than rationals.

Definition 4.1.3. A complex number is said to be definable if there is an abstract $A$ such that the complex is expressed as $\{x\} A(x)$.

Definition 4.1.4. Sequences of complex numbers. The sequences are defined as in Chapter 3. Namely a sequence of complex numbers is defined by an abstract (in some parameters) which defines it. Let $A(n, x)$ be a formula such that for every $n(i . e ., N(n)) C(\{x\} A(n, x))$. Then we say that $A$ defines a sequence, which we express as $\left\{a_{n}\right\}_{n}$. 
Proposition 4.1.9. (1) The finite sum (of a definable sequence) is definable.

(2) The series (of a definable sequence) is definable.

Proof. Let $\left\{a_{n}\right\}_{n}$ be a definable sequence. Let $\operatorname{Re}\left(\alpha_{n}\right)=a_{n}$ and $\operatorname{Im}\left(\alpha_{n}\right)=b_{n}$.

(1) $\sum_{n=1}^{m} \alpha_{n}=\sum_{\mathrm{df}}^{m} a_{n}+i \sum_{n=1}^{m} b_{n}$, where $\sum a_{n}$ and $\sum b_{n}$

are defined arithmetically (Remark after Proposition 4.1.5; recall that $\operatorname{Re}\left(\alpha_{n}\right)$ and $\operatorname{Im}\left(\alpha_{n}\right)$ are arithmetical in $\left.\alpha_{n}\right)$.

(2) $\sum_{n=1}^{\infty} \alpha_{n}=\left(\limsup _{n} \sum_{i=1}^{n} a_{i}\right)+i\left(\liminf _{n} \sum_{i=1}^{n} b_{i}\right)$.

A series is said to be convergent if $\lim \sup =\lim$ inf $=\lim$.

Proposition 4.1.10. The finite product of a definable sequence is definable.

Proof. Let $\left\{\alpha_{n}\right\}_{n}$ be a sequence where $\alpha_{n}=a_{n}+i b_{n}$. The sequences $\left\{a_{n}\right\}$ and $\left\{b_{n}\right\}$ are definable from $\left\{a_{n}\right\}$. Consider an example, $\alpha_{1} \cdot \alpha_{2} \cdot \alpha_{3} \cdot \alpha_{4}$. We know, in arithmetic

$$
\begin{aligned}
\alpha_{1} \cdot \alpha_{2} \cdot \alpha_{3} \cdot \alpha_{4}= & a_{1} a_{2} a_{3} a_{4}+i\left[a_{1} a_{2} a_{3} b_{4}+a_{1} a_{2} b_{3} a_{4}+a_{1} b_{2} a_{3} a_{4}+b_{1} a_{2} a_{3} a_{4}\right]+ \\
& i^{2}\left[a_{1} a_{2} b_{3} b_{4}+a_{1} b_{2} a_{3} b_{4}+a_{1} b_{2} b_{3} a_{4}+b_{1} a_{2} a_{3} b_{4}+b_{1} a_{2} b_{3} a_{4}+b_{1} b_{2} a_{3} a_{4}\right]+ \\
& i^{3}\left[a_{1} b_{2} b_{3} b_{4}+b_{1} a_{2} b_{3} b_{4}+b_{1} b_{2} a_{3} b_{4}+b_{1} b_{2} b_{3} a_{4}\right]+ \\
& i^{4}\left[b_{1} b_{2} b_{3} b_{4}\right] .
\end{aligned}
$$

If we denote this in an abbreviated expression,

$$
=[0]+i[1]+i^{2}[2]+i^{3}[3]+i^{4}[4],
$$

we can generalize the expression to an arbitrary $n$ :

$$
(*) \quad \alpha_{1} \cdot \alpha_{2} \cdots \alpha_{n}=\prod_{i=1}^{n} \alpha_{i}=[0]+i[1]+\cdots+i^{k}[h]+\cdots+i^{n}[n] .
$$

where $[k]=\sum_{i=1}^{(n k)} \prod_{j=1}^{n} c_{j}, c_{j}=a_{j}$ or $b_{j}$ and there are $k$ of the $b_{j}$ 's. In tidying up the right hand side of $(*)$, using $i^{2}=-1$, we need to use a case by case definition; $n \equiv 0,1,2,3(\bmod 4)$. Suppose, as an example, $n \equiv 0(\bmod 4)$. Then $i^{n}=1$. So

$$
\begin{aligned}
& \operatorname{Re}\left(\prod_{i=1}^{n} \alpha_{i}\right)=[0]-[2]+[4]-\cdots+[n] \\
& \operatorname{Im}\left(\prod_{i=1}^{n} \alpha_{i}\right)=[1]-[3]+[5]-\cdots-[n-1] .
\end{aligned}
$$


Thus, it is obvious that $[k]$ is definable (depending on $\left\{\alpha_{i}\right\}$ and $n$ ) and $\prod_{i=1}^{n} \alpha_{i}$ is definable (as a function of $n$ ).

Corollary 4.1.11. The powers in $\alpha, \alpha^{n}$ (as a function of $n$ ) form a definable function.

Proposition 4.1.12. Let $\left\{\alpha_{n}\right\}_{n}$ be definable. Then $\lim _{n} \alpha_{n}$ is definable.

Proof. Define $\limsup \alpha_{n}=\limsup a_{n}+i\left(\limsup b_{n}\right)$, where $\alpha_{n}=a_{n}+i b_{n}$, $\lim \sup a_{n}$ and $\lim \sup b_{n}$ are definable (Chapter 3), hence the right hand side is definable. We define $\lim \inf \alpha_{n}$ similarly. If $\lim \sup \alpha_{n}=\lim \inf \alpha_{n}$ and each defines a complex number, that we call $\lim \alpha_{n}$. Furthermore $\lim \alpha_{n}$ is definable.

Proposition 4.1.13. $\lim \alpha_{n}=\beta$ if and only if $\forall \varepsilon>0 \exists N \forall n \geq N\left(\left|\alpha_{n}-\beta\right|<\varepsilon\right)$ where $\varepsilon$ ranges over positive (real) rational.

Definition 4.1.5. A complex sequence $\left\{\alpha_{n}\right\}_{n}$ is said to be Cauchy if

$$
\forall \varepsilon>0 \exists N \forall n, m \geq N\left(\left|\alpha_{n}-\alpha_{m}\right|<\varepsilon\right) .
$$

Proposition 4.1.14. The sequence $\left\{\alpha_{n}\right\}$ is Cauchy if and only if $\left\{a_{n}\right\}$ and $\left\{b_{n}\right\}$ are Cauchy (as real sequences) where $\alpha_{n}=a_{n}+i b_{n}$.

If $\lim \alpha_{n}$ exists, then we say that $\left\{\alpha_{n}\right\}$ is convergent. If $\lim \alpha_{n}=\beta$, then we say that $\left\{\alpha_{n}\right\}$ converges to $\beta$.

Proposition 4.1.15. The sequence $\left\{\alpha_{n}\right\}$ is convergent if and only if it is Cauchy.

Proof. The sequence $\left\{\alpha_{n}\right\}$ is convergent if and only if $\left\{a_{n}\right\}$ and $\left\{b_{n}\right\}$ are convergent. So, by Proposition 4.1.14, the problem is reduced to the case of real sequences, which was proved in Chapter 3.

Proposition 4.1.16. (1) The series $\sum \alpha_{n}$ is convergent if and only if $\forall \varepsilon>$ $0 \exists N \forall n \geq N \forall p\left(\left|\sum_{n}^{n+p} \alpha_{n}\right|<\varepsilon\right)$. Therefore $\left\{\alpha_{n}\right\}$ converges to 0 if $\sum \alpha_{n}$ is convergent.

(2) If $\sum\left(\alpha_{n}\right)$ converges, then $\sum \alpha_{n}$ is also absolutely convergent. (It is obvious that if $\left\{\alpha_{n}\right\}$ is definable, then $\left\{\left|\alpha_{n}\right|\right\}$ is also.)

\subsection{Analytic functions}

Definition 4.2.1. 1) A (definable) domain is an arithmetical abstract of type $2 ;\{\varphi\} D(\varphi)$, such that $\forall \varphi(D(\varphi) \supset C(\varphi))$. We write $\varphi \in D$ if $D(\varphi)$.

2) $A$ (complex) function defined on $D$ is an abstract $\{\varphi, x\} A(\varphi, x)$ such that $\forall \varphi \in D C(\{x\} A(\varphi, x))$, where $x$ is of type 0 . We write $f(\gamma)=\{x \mid A(\gamma, x)\}$. 
In a similar manner we can define functions from "reals" to "reals", from "reals" to complex numbers etc.

Definition 4.2.2. 1) Let $f$ be a (complex) function defined in D. Suppose $\alpha \in D$. We say that $f$ is continuous at $\alpha$ if

$$
\forall \varepsilon>0 \exists \delta>0 \forall \varphi \in D(|\varphi-\alpha|<\delta \supset|f(\varphi)-f(\alpha)|<\varepsilon)
$$

where $\varepsilon$ and $\delta$ stand for rational reals and || is the absolute value function.

2) We can define the notion that $f$ is continuous "around $\alpha$ " naturally.

3) The functions, $\operatorname{Re} f$ and $\operatorname{Im} f$, whose values are the real part of $f$ and the imaginary part of $f$ (hence the values are "reals") respectively, are definable. We can define continuity of such functions in a similar manner as in 1).

As in Chapter 3, we can show that the composition of functions is definable and the composition of continuous functions is continuous.

Proposition 4.2.1. A function $f$ is continuous at $\alpha$ if and only if $\operatorname{Re} f$ and $\operatorname{Im} f$ are continuous at $\operatorname{Re}(\alpha)$ and $\operatorname{Im}(\alpha)$ respectively.

Proposition 4.2.2. Suppose $f$ is continuous around $\alpha$. Then $\lim \sup f(\varphi)$ and $\liminf _{\varphi \rightarrow \alpha} f(\varphi)$ are definable.

If two limits are equal, then we write either one as $\lim _{\varphi \rightarrow \alpha} f(\varphi)$.

Proof. Let $g=\operatorname{Re} f$ and $h=\operatorname{Im} f$. We define $\lim \sup g$ and $\lim \inf g$ by

$$
\begin{gathered}
\limsup _{\varphi \rightarrow \alpha} g(\varphi)=* \bigcap_{\varepsilon>0} \bigcup_{0 \neq|a|<\varepsilon} g(\alpha+a) \text { and } \\
\liminf _{\varphi \rightarrow \alpha} g(\alpha)={ }^{*} \bigcup_{\varepsilon>0} \bigcap_{0 \neq|a|<\varepsilon} g(\alpha+a)
\end{gathered}
$$

respectively, where $a$ denotes a complex rational. Then we define $\lim \sup f$ and $\liminf f$ by

$$
\limsup _{\varphi \rightarrow \alpha} f(\varphi)=\limsup _{\varphi \rightarrow \alpha} g(\varphi)+i \limsup _{\varphi \rightarrow \alpha} h(\varphi)
$$

and

$$
\liminf _{\varphi \rightarrow \alpha} f(\varphi)=\liminf _{\varphi \rightarrow \alpha} g(\varphi)+i \liminf _{\varphi \rightarrow \alpha} h(\varphi)
$$

respectively.

Proposition 4.2.3. 1) $\lim _{\varphi \rightarrow \alpha} f(\varphi)=\beta$ if and only if

$$
\forall \varepsilon>0 \exists \delta>0 \forall \varphi \in D(|\varphi-\alpha|<\delta \supset|f(\varphi)-\beta|<\varepsilon) .
$$


2) $\lim f(\varphi)=\beta$ if and only if $\lim g=\operatorname{Re} \beta$ and $\lim h=\operatorname{Im} \beta$; where $g=\operatorname{Re} f$ and $h=\operatorname{Im} f$.

3) $\lim f=\beta$ if and only if $\lim \bar{f}=\bar{\beta}$ where $\bar{f}$ is defined by $\bar{f}(\alpha)=\operatorname{Re}(f(\alpha))-$ $i \operatorname{Im}(f(\alpha))$.

Definition 4.2.3. The derivative of $f(\alpha)$ is defined by $f^{\prime}(\alpha)=\limsup _{\varphi \rightarrow \alpha} \frac{f(\varphi)-f(\alpha)}{\varphi-\alpha}$.

If this limit exists and $f$ is continuous, then we may say that the derivative exists at $\alpha$, or $f$ is differentiable at $\alpha$.

Definition 4.2.4. A complex function which is differentiable everywhere in a domain is called analytic. We follow [1] in the terminology. "Analytic" is usually called "regular" or "holomorphic".

It is obvious that analytic functions are closed under $+,-, \cdot, \div$.

Proposition 4.2.4. Let $f$ be analytic. Partial derivatives $\frac{\partial f}{\partial x}$ and $\frac{\partial f}{\partial y}$ are definable.

Proof. Let $z=x+i y$. Then $\frac{\partial f}{\partial x}$ is defined to be $\limsup _{\substack{h \rightarrow 0 \\ R(h)}} \frac{f(\alpha+h)-f(\alpha)}{h}$, where $\alpha+h=(a+h)+i b$. If $\lim \sup =\liminf ($ hence $=\lim )$, we say that the partial derivative exists. Here $h \underset{R(h)}{\rightarrow} 0$ means to take "real" $h$ only. Since this can be replaced by "rational reals", the derivative is definable. Similarly $\frac{\partial f}{\partial y}=\lim _{\substack{h \rightarrow 0 \\ R(h)}} \frac{f(\alpha+i h)-f(\alpha)}{h}$.

Proposition 4.2.5. Let $f=u+i v$ where $u=\operatorname{Re} f$ and $v=\operatorname{Im} f$. Let $z=x+i y$. Then

$$
\frac{\partial f}{\partial x}=\frac{\partial u}{\partial x}+i \frac{\partial v}{\partial x} \text { and } \frac{\partial f}{\partial y}=\frac{\partial u}{\partial y}+i \frac{\partial v}{\partial y} .
$$

As a consequence, the Cauchy-Riemann differential equations for analytic functions hold, namely $\frac{\partial u}{\partial x}=\frac{\partial v}{\partial y}$ and $\frac{\partial u}{\partial y}=-\frac{\partial v}{\partial x}$.

Definition 4.2.5. A domain $D$ is said to be open if

$$
\forall \varphi \in D \exists r>0 \forall \psi(|\varphi-\psi|<r \supset \psi \in D)
$$

where $r$ stands for a rational real.

Proposition 4.2.6. Let $f=u+i v$ be defined on an open domain $D$, where $u=\operatorname{Re} f$ and $v=\operatorname{Im} f$. Let $z=x+i y$. If $u$ and $v$ have continuous first order partial derivatives in $D$ and satisfy the Cauchy-Riemann differential equation in $D$, then $f$ is analytic in $D$. 
We can also prove Laplace's equation and that $\left|f^{\prime}(z)\right|^{2}$ is the Jacobian of $u$ and $v$. We can define the notion of "harmonic" for definable (real-valued) complex variable functions.

Definition 4.2.6. A sequence of functions (complex) $\left\{f_{n}(z)\right\}_{n}$ is said to be definable if there is an arithmetical formula $A(n, r, z)$ such that $f_{n}(z)=\{r\} A(n, r, z)$ and $\forall n \forall z \in D(C(\{r\} A(n, r, z))$, where $D$ is a definable domain of complex numbers.

Proposition 4.2.7. If $\left\{f_{n}\right\}$ is a sequence of functions, then the limit of the sequence and the series of the sequence are definable. See Chapter 3 for details.

As a special case of function series, we can define power series: Let $\left\{\alpha_{n}\right\}$ be a sequence of complex numbers. The definition of the finite products in Proposition 4.1 .10 can be employed for $\alpha_{n}=z$ there (where $z$ is available). Therefore $\left(\alpha_{n} z^{n}\right)$ is a sequence of complex functions. We call $\sum \alpha_{n} z^{n}$ a power series with coefficient sequence $\left\{\alpha_{n}\right\}$.

Proposition 4.2.8. The radius of convergence (Abel) of a power series is definable.

Proof. Let $\left\{\alpha_{n}\right\}$ be the sequence of coefficients of the power series. Then $\left\{\left|\alpha_{n}\right|\right\}$ is definable, hence $\left\{\sqrt[n]{\left|\alpha_{n}\right|}\right\}$ is definable. Therefore limsup $\sqrt[n]{\left|\alpha_{n}\right|}$ is definable. We know that lim exists and it gives the radius of convergence.

\subsection{Integration}

From now on, we do not make any distinction between free variables and bound variables since we have a shortage of letters. Let $z, z_{1}, z_{2}, \ldots$ stand for complex numbers, that is, $\forall z$ is an abbreviation of $\forall \alpha(C(\alpha) \supset \cdots)$. Let $\mathbb{R}$ and $\mathbb{C}$ denote the set of all real numbers and the set of all complex numbers respectively.

Definition 4.3.1. 1) An ordered triple $(\pi, a, b)$ is called an arc if $a<b$ and $\pi:[a, b] \rightarrow \mathbb{C}$ is continuous. We simply say that $\pi$ is an arc.

2) An ordered quintuple $(\pi, a, b, n, h)$ is called a piecewise differentiable arc if $(\pi, a, b)$ is an arc, $h:\{0, \ldots, n\} \rightarrow[a, b)$ is a strictly increasing function with $h(0)=a$, and $\pi$ is differential on $\left[a_{i}, a_{i+1}\right](i=0, \ldots, n)$, where $a_{i}=h(i)$ and $a_{n+1}=b$, and by the differentiability of $\pi$, we mean that $\pi^{\prime}$ exists and is continuous. Usually we say that $\left(\pi, a=a_{0}<a_{1}<\right.$ $\left.\ldots<a_{n}<b\right)$ is a differentiable arc. We denote differentiable arcs by $\gamma, \gamma_{1}, \gamma_{2}, \ldots$. Therefore $\forall \gamma$ stands for

$$
\forall \pi \forall a \forall b \forall n \forall h(A(\pi, a, b, n, h) \supset
$$

where $A(\pi, a, b, n, h)$ is the condition on $\pi, a, b, n, h$ in the definition of differentiable arc. 
We also use the convention of denoting $\pi(t)$ by $z(t)$ and its real part and imaginary part by $x(t)$ and $y(t)$ respectively. Therefore $\pi(t)$ is expressed by

$$
z(t)=x(t)+i y(t)
$$

3) We define $\int_{\gamma} f(z) d z$ by

$$
\int_{\gamma} f(z) d z=\int_{a}^{b} f(z(t)) z^{\prime}(t) d t
$$

where $\gamma=(\pi, a, b, n, h)$ and $\pi$ is expressed by $z$.

Let $\gamma_{1}, \ldots, \gamma_{n}$ be piecewise differential arcs. We take the usual definition of $-\gamma_{1}$ and $\gamma_{1}+\cdots+\gamma_{n}$. The properties of $\int_{\gamma}$ are easily proved e.g.

$$
\begin{gathered}
\int_{-\gamma} f(z) d z=-\int_{\gamma} f(z) d z \text { and } \\
\int_{\gamma_{1}+\cdots+\gamma_{n}} f d z=\int_{\gamma_{1}} f d z+\cdots+\int_{\gamma_{n}} f d z .
\end{gathered}
$$

4) Let $\gamma=(\pi, a, b, n, h)$ be a piecewise differentiable arc and let $p(x, y)$ be a continuous function of real parameters $x$ and $y$. We define $\int_{\gamma} p(x, y) d x$ and $\int_{\gamma} p(x, y) d y$ by the following equations

$$
\begin{gathered}
\int_{\gamma} p(x, y) d x=\int_{a}^{b} p(x(t), y(t)) x^{\prime}(t) d t \quad \text { and } \\
\int_{\gamma} p(x, y) d y=\int_{a}^{b} p(x(t), y(t)) y^{\prime}(t) d t
\end{gathered}
$$

where $\pi$ is denoted by $z, x$ is $\operatorname{Re} z$, and $y$ is $\operatorname{Im} z$, that is,

$$
z(t)=x(t)+i y(t)
$$

5) An arc $(\pi, a, b)$ is said to be linear if

$$
\forall t \in[a, b] \pi(t)=\frac{1}{b-a}((b-t) \pi(a)+(t-a) \pi(b)) .
$$

Let $z_{1}$ and $z_{2}$ be two complex numbers, we define $z_{1} \square z_{2}$ by

$$
z_{1} \square z_{2} \quad \text { iff } \operatorname{Re} z_{1}=\operatorname{Re} z_{2} \vee \operatorname{Im} z_{1}=\operatorname{Im} z_{2} .
$$


A piecewise differentiable arc $\left(\pi, a=a_{0}<a_{1}<\cdots<a_{n}<b\right)$ is said to be a rectilinear polygon if for every $i \leq n, \pi$ is linear on $\left[a_{i}, a_{i+1}\right]$ and $\pi\left(a_{i}\right) \square \pi\left(a_{i+1}\right)$.

6) An ordered triple $\left(D, z_{0}, \Pi\right)$ is said to be a region if $D$ is an open domain, $z_{0} \in D$, and for every $z \in D, \Pi(z)$ is a rectilinear polygon in $D$ from $z_{0}$ to $z$. Precisely $\Pi$ is an ordered quintuple of functions, that is $\Pi=\left(\Pi_{1}, \Pi_{2}, \ldots, \Pi_{5}\right)$. We will let $\Omega, \Omega_{0}, \Omega_{1}, \ldots$ stand for regions, that is, $\forall \Omega$ is an abbreviation of $\forall D \forall z_{0} \forall \Pi\left(A\left(D, z_{0}, \Pi\right) \supset\right.$ where $A\left(D, z_{0}, \Pi\right)$ expresses the condition on $D, z_{0}, \Pi$, for regions and $\forall \Pi$ is an abbreviation for $\forall \Pi_{1} \cdots \forall \Pi_{5}$.

In our work, we usually give only $D$ for $\Omega$ since the construction of $\Pi$ is immediate and automatic as is easily seen in the following examples.

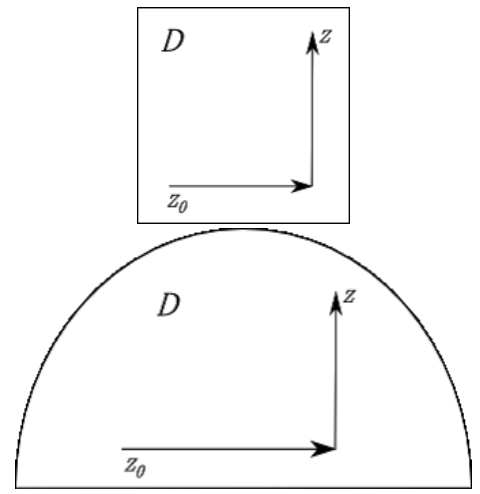

Theorem 4.3.1. The line integral $\int_{\gamma} p d x+q d y$, defined in $\Omega$, depends only on the end points of $\gamma$ iff there exists an abstract $U(x, y)$ such that $U(x, y)$ is a function in $\Omega$ with partial derivatives $\frac{\partial U}{\partial x}=p, \frac{\partial U}{\partial y}=q$.

Proof. The sufficiency follows at once. To prove the necessity, let $\Omega=\left(C, z_{0}, \Pi\right)$ and $U(x, y)=\int_{\gamma} p d x+q d y$, where $\gamma=\Pi(z)$ and $z=x+i y$.

Corollary 4.3.2. The integral $\int_{\gamma} f d z$, with continuous $f$, depends only on the end points of $\gamma$ iff $f$ is a derivative of an analytic function in $\Omega$.

From the corollary it follows that

$$
\int_{\gamma}(z-a)^{n} d z=0
$$

for $n \neq-1$ and for all closed curves $\gamma$ which do not pass through $a$. 


\subsection{Cauchy's Theorem for nice regions}

Let $\Omega$ be a region and let a rectangle $R$ be defined by the inequalities $a \leq x \leq$ $b, c \leq y \leq d$ in $\Omega$. The perimeter of $R$ is directed in the order $(a, c),(b, c),(b, d),(a, d)$. We refer to this closed curve as the boundary curve of $R$, and we denote it by $\Gamma(R)$.

Theorem 4.4.1. If $f(z)$ is analytic in $\Omega$, then

$$
\int_{\Gamma(R)} f(z) d z=0
$$

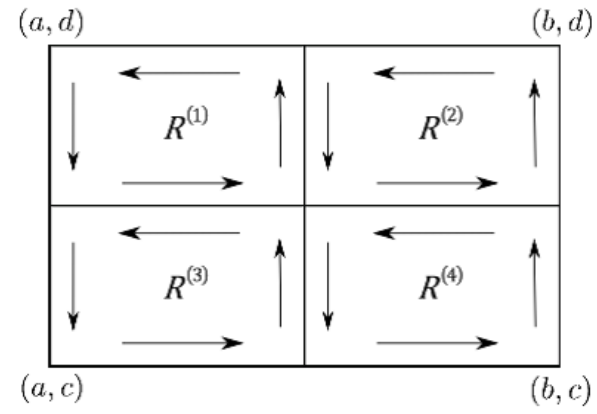

Proof. The proof follows the usual method of bisection. We let

$$
\eta(R)=\int_{\Gamma(R)} f(z) d z .
$$

If $R$ is divided into four congruent rectangles $R^{(1)}, R^{(2)}, R^{(3)}, R^{(4)}$, then

$$
\eta(R)=\eta\left(R^{(1)}\right)+\eta\left(R^{(2)}\right)+\eta\left(R^{(3)}\right)+\eta\left(R^{(4)}\right) .
$$

We continue dividing each $R^{(k)}(k=1, \ldots, 4)$ into four congruent rectangles, get

$$
R^{(5)}, \ldots, R^{(20)}
$$

and keep going on. Thus we get on $R^{(n)}$ for every $n=1,2,3, \ldots$. This allows us to use $n$ in the place of $R^{(n)}$ and we can calculate arithmetically all vertexes of $R^{(n)}$ in terms of $n$. Since we can quantifier on $n$, we can carry out the proof very easily. As usual we choose $R_{1}$ among $R^{(1)}, \ldots, R^{(4)}$ such that

$$
\left|\eta\left(R_{1}\right)\right| \geq \frac{1}{4}|\eta(R)|
$$

We repeat this procedure and get $R_{1}, R_{2}, R_{3}, \ldots$ such that $R_{i+1}$ is one of four subrectangles of $R_{i}$ such that

$$
\left|\eta\left(R_{i+1}\right)\right| \geq \frac{1}{4}\left|\eta\left(R_{i}\right)\right| .
$$

Choosing the first one with this condition among $R^{(1)}, R^{(2)}, \ldots$ as the $R_{i+1}$, we can carry out everything arithmetically, for example, $\eta\left(R_{i}\right)$ is uniformly defined as an abstract with a parameter $i$. So we have proved it. 
Let $\Omega$ be a region. Whenever we talk about a finite number of points in $\Omega$, we mean that $n$ and $h:\{0, \ldots, n\} \rightarrow \Omega$ are given. Note that $\{0, \ldots, n\}$ is definable as an abstract.

Theorem 4.4.2. Let a rectangle $R$ be in $\Omega$ and let $f(z)$ be analytic in $\Omega$ except at a finite number of interior points, $\zeta_{j}$, of $R$. If $\lim _{z \rightarrow \zeta_{j}}\left(z-\zeta_{j}\right) f(z)=0$ for all $j$, then

$$
\int_{\Gamma(R)} f(z) d z=0
$$

Proof. Since $\zeta_{0}, \ldots, \zeta_{n}$ are given by an abstract, we can arithmetically divide $R$ into smaller rectangles which contain at most one $\zeta_{j}$. After this, it is proved in the usual way.

Let $\Delta$ be an open circular disk $\left|z-z_{0}\right|<e$. We can easily construct $z_{0}, \Pi$ so that $\left(\Delta, z_{0}, \Pi\right)$ is a region. By the usual proof, we have the following theorems.

Theorem 4.4.3. If $f(z)$ is analytic in $\Delta$, then

$$
\int_{\gamma} f(z) d z=0
$$

for every closed curve $\gamma$ in $\Delta$.

Theorem 4.4.4. Let $f(z)$ be analytic in the region $\Delta^{\prime}$, obtained by omitting a finite number of points $\zeta_{j}$ from $\Delta$. If $f(z)$ satisfies the condition $\lim _{z \rightarrow \zeta_{j}}(z-$ $\left.\zeta_{j}\right) f(z)=0$ for all $j$, then

$$
\int_{\gamma} f(z) d z=0
$$

holds for any closed curve $\gamma$ in $\Delta$.

\subsection{Cauchy's Integral Formula}

Let $\gamma$ be a piecewise differentiable closed curve which does not pass through the point $\alpha$. Then the value of the integral

$$
\int_{\gamma} \frac{d z}{z-\alpha}
$$

is a multiple of $2 \pi i$.

This is proved in the usual way. That is, if

$$
h(t)=\int_{a}^{t} \frac{z^{\prime}(t)}{z(t)-\alpha} d t
$$

where $\gamma$ is expressed by $\left(z, a=a_{0}<a_{1}<\cdots<b\right)$, then $h^{\prime}(t)=\frac{z^{\prime}(t)}{z(t)-a}$ is continuous whenever $z^{\prime}(t)$ is continuous. Therefore $\frac{d}{d t}\left(e^{-h(t)}(z(t)-\alpha)\right)$ vanishes 
except at a finite number of points. Since $e^{-h(t)}(z(t)-\alpha)$ is continuous, it is a constant and

$$
e^{h(t)}=\frac{z(t)-\alpha}{z(a)-\alpha}
$$

Since $z(b)=z(a)$, we have $e^{h(b)}=1$ and $h(b)$ is a multiple of $2 \pi i$. Obviously everything can be carried out in our system FA.

We let

$$
n(\gamma, \alpha)=\frac{1}{2 \pi i} \int_{\gamma} \frac{d z}{z-\alpha}
$$

and call $n(\gamma, \alpha)$ the index of $\alpha$ with respect to $\gamma$.

Many properties of the index can be proved in FA by the usual method. Especially, if $\gamma$ lies inside of a circle, then $n(\gamma, a)=0$ for all points $a$ outside of the same circle. Also if the line segment $\overline{a b}$ and $\gamma$ are disjoint, then $n(\gamma, a)=$ $n(\gamma, b)$.

We can carry out everything in Chapter 3 in [1] in FA almost word by word. However there are a few things which we have to change. Whenever each of the regions determined by $\gamma$ is discussed in [1], we have to change it to a more restricted situation of a region $\Omega$ such that the point $z_{0}$ of $\Omega$ has non-zero index with respect to $\gamma$. This is necessary since ' $\Omega$ is determined by $\gamma$ ' is not definable in FA. However we do not need this complicated notion in practice. In every practical case, $\Omega$ and $\gamma$ are given from the beginning and it is obvious that $\Omega$ is determined by $\gamma$. Besides all necessary cases are covered by the following proposition which is Lemma 2 in Chapter 3 of [1] and proved in the same way.

Proposition 4.5.1. Let $z_{1}, z_{2}$ be two points on a closed curve $\gamma$ which does not pass through the origin. Denote the subarc from $z_{1}$ to $z_{2}$ in the direction of the curve by $\gamma_{1}$, and the subarc from $z_{2}$ to $z_{1}$ by $\gamma_{2}$. Suppose that $z_{1}$ lies in the lower half plane and $z_{2}$ in the upper half plane. If $\gamma_{1}$ does not meet the negative real axis and $\gamma_{2}$ does not meet the positive real axis, then $n(\gamma, 0)=1$.

Note that by a curve we mean a piecewise differentiable arc.

Thus we have Cauchy's integral formula in the form

$$
n(\gamma, a) \cdot f(a)=\frac{1}{2 \pi i} \int_{\gamma} \frac{f(z) d z}{z-a}
$$

if $f$ is analytic in an open dish $\Delta$ and $\gamma$ is a closed curve in $\Delta$.

Then

$$
f^{(n)}(z)=\frac{n !}{2 \pi i} \int_{C} \frac{f(\zeta) d z}{(\zeta-z)^{n+1}}
$$

where $f$ is analytic in $\Omega, C$ is a circle whose center is $z$, and $C$ and the open disk determined in $C$ are in $\Omega$.

This gives us a simpler expression for $f^{(n)}$ by an abstract. Thus we can carry out the usual calculations about line integration, differentiation, and limits of sequences in FA. Consequently we have the theorem on removable singularities and Taylor's theorem. 
However when topology is discussed in complex analysis, we have to pay special attention since many simple arguments in topology cannot be carried out in FA as they are. The first such case comes in the following theorem.

Theorem 4.5.2. Let $f$ be analytic in a region $\Omega$ and let $a \in \Omega$. If $f(a)$ and all derivatives $f^{(n)}(a)$ vanish, then $f(z)$ is identically zero in all of $\Omega$.

Proof. As usual, we can show that $f(z)$ is identically zero in a neighborhood of $a$. Since $\Omega$ is a region, it suffices to show that $f(b)=0$ if the line segment $\overline{a b}$ is in $\Omega$. Without loss of generality we assume that $a=0, b=1$ and $[0,1]$ is in $\Omega$.

First we define a real $c$ by

$c={ }^{*}\left\{r \mid 0 \leq r<1 \wedge \forall s \in[0, r]\left(f(s)\right.\right.$ and all derivative $f^{(n)}(s)$ vanish $\left.)\right\}$.

Obviously we have $0 \leq c \leq 1$. Since $f$ is identically zero in a neighborhood of 0 , we have $0<c$. Take any real $d$ with $0<d<c$. First we claim that $f(d)$ and all derivative $f^{(n)}(d)$ vanish. Suppose not, that is, suppose there exists on $n$ such that

$$
f^{(n)}(d) \neq 0
$$

Since $f^{(n)}$ is continuous at $d$, there exists an $\varepsilon>0$ such that $\forall r(|r-d|<\varepsilon \supset$ $\left.f^{(n)}(r) \neq 0\right)$. But this contradicts the definition of $c$.

By the same argument we can show that $f(c)$ and all derivative $f^{(n)}(c)$ vanish. Therefore $c$ should be pushed further right if $c<b$. Consequently $b=c$.

From this theorem, we can prove that the zero of an analytic function $f$ are isolated if $f$ is not identically zero. Therefore we can prove the following theorem.

Theorem 4.5.3. Let $\Omega$ be a region and let $f$ and $g$ be analytic in $\Omega$ with $f(z)=g(z)$ on a set which has an accumulation point in $\Omega$. Then $f(z)$ is identically equal to $g(z)$ in $\Omega$.

Therefore $f(z)$ is identically zero in $\Omega$, if $f(z)$ vanishes on an arc which does not reduce to a point. However the following form of the theorem must be carefully stated.

If $f(z)=0$ on a bounded infinite set in $\Omega$, then $f(z)$ is identically zero in $\Omega$. In order to state this precisely, we define

$$
\mathbb{N}=\{n \mid N(n)\}
$$

A set $A$ is said to be infinite if there exists a one-to-one map $h$ from $\mathbb{N}$ into $A$. Then our theorem is as follows.

Theorem 4.5.4. Let $\Omega$ be a region and let $f$ be analytic in $\Omega$. If $R$ is a rectangle inside $\Omega$ and $f$ vanishes on an infinite subset of $R$, then $f(z)$ is identically zero in $\Omega$. 
Proof. Assume that $f(z)$ vanishes at distinct points $a_{0}, a_{1}, a_{2}, \ldots$ in $R$. By using this enumeration, we can use the bisection methods as in the proof of Cauchy's theorem in $\S 4$ and construct an accumulation point of $a_{0}, a_{1}, a_{2}, \ldots$ as an abstract.

As is seen in these theorems, it is important to enumerate the zeros of $f$ in $\Omega$. This is done in the following theorem.

Theorem 4.5.5. Let $\Omega$ be a region and let $f$ be analytic in $\Omega$. Then we can construct a function $h$, as an abstract, to enumerate all zeros of $f$.

Proof. First fix an enumeration $r_{0}, r_{1}, r_{2}, \ldots$ of all complex rational numbers. Then enumerate all $(r, \varepsilon)$ such that $r$ is a complex rational and $\varepsilon>0$ with

$$
\{s|| r-s \mid<\varepsilon\} \subseteq \Omega .
$$

In each such $(r, \varepsilon)$, consider the $\varepsilon$-neighborhood of $r$, denoted by $U(r, \varepsilon)$. In $U(r, \varepsilon)$, define $s_{1}, s_{2}, \ldots$ by the following condition.

Let $s_{i}$ be the first in the enumeration of the rationals such that

$$
s_{i} \in U(r, \varepsilon) \wedge\left|f\left(s_{i}\right)\right|<\frac{1}{2^{i}} .
$$

We take $a=\lim _{i \rightarrow \infty} s_{i}$ if it exists and check whether or not $a \in \Omega$ and $f(a)=0$. If it is the case, we take $a$. The whole procedure is arithmetic and the theorem is proved.

By using this theorem, the following theorem is proved in the usual manner.

Theorem 4.5.6. Let $f$ be analytic in a circular disk $\Delta$ and be not identically zero in $\Delta$. Let $z_{j}$ be the zeros of a function $f(z)$, each zero being counted as many times as its order indicates. For every closed curve $\gamma$ in $\Delta$ which does not pass through a zero

$$
\sum_{j} n\left(\gamma, z_{j}\right)=\frac{1}{2 \pi i} \int_{\gamma} \frac{f^{\prime}(z)}{f(z)} d z
$$

where the sum has only a finite number of terms $\neq 0$.

We can also prove the properties of poles, Weierstrass' theorem on an essential singularity, and the local properties of analytic functions. The following form of the maximum principle is proved in the usual manner, too.

Theorem 4.5.7. If $f(z)$ is analytic and nonconstant in a region $\Omega$, then its absolute value $|f(z)|$ has no maximum in $\Omega$.

However in order to prove a better form of the maximum principle, we need some preparation in topology.

Theorem 4.5.8. Let $D$ be an open set. Then the closure of $D$, denoted by $\bar{D}$, is definable as an abstract. 
Proof. We let

$$
\bar{D}=\{z|\forall \varepsilon>0 \exists r \in D| z-r \mid<\varepsilon\}
$$

where $r$ stands for a complex rational.

Theorem 4.5.9. Let $D$ be open and bounded. If $f: \bar{D} \rightarrow \mathbb{R}$ is continuous, then $f$ attains its maximum in $\bar{D}$.

Proof. First we let

$$
M={ }^{*}\{r \mid \exists s \in D r<f(s)\}
$$

where $s$ stands for a complex rational and $r$ stands for a real rational. Obviously $M$ is the least upperbound of $f$. Fix an enumeration $r_{0}, r_{1}, r_{2}, \ldots$ of all complex rationals. Define $s_{1}, s_{2}, \ldots$ as follows.

Let $s_{i}$ be the first complex rational in the enumeration such that

$$
s_{i} \in D \wedge M<f\left(s_{i}\right)+\frac{1}{i} .
$$

Then $s_{1}, s_{2}, \ldots$ is a sequence in a rectangle. As in the proof of Theorem 4.5.4, we can construct an accumulation point of $s_{1}, s_{2}, \ldots$ as an abstract by the bisection method. Let $s$ be an accumulation point. Then we have $s \in \bar{D} \wedge f(s)=M$.

By this theorem, we have the following form of the maximum principle.

Theorem 4.5.10. If $f(z)$ is analytic in a bounded region $\Omega$ and continuous in $\bar{\Omega}$, then the maximum of $|f(z)|$ is taken on the boundary of $\Omega$.

We can also prove the lemma of Schwarz.

\subsection{The general form of Cauchy's Theorem}

Since we would like to avoid topological arguments as much as possible, we take the following definition of simple connectedness.

Definition 4.6.1. Let $\Omega$ be a region. Then $\Omega$ is said to be simply connected if $n(\gamma, a)=0$ for all closed curve $\gamma$ in $\Omega$ and all points a which do not belong to $\Omega$.

This definition of simple connectedness is sufficient for all practical purposes since in all the practical cases it is easy to show simple connectedness according to the foregoing definition.

This definition allows us to follow the proofs of $\S 4$ and 5 in Chapter 3 in [1] word by word and we have the following theorem.

Theorem 4.6.1. If $f(z)$ is analytic in a simply connected region $\Omega$, then

$$
\int_{\gamma} f(z) d z=0
$$

for every closed curve $\gamma$ in $\Omega$. 
Corollary 4.6.2. If $f(z)$ is analytic and $\neq 0$ is a simply connected region $\Omega, a$ single-valued analytic branch of $\log f(z)$ can be defined in $\Omega$.

We can also prove the Residue Theorem and its applications in Chapter 3 in $[1]$.

\subsection{Miscellaneous remarks}

The theorem of uniform continuity of a continuous function on a rectangle in Chapter 3 can be generalized as follows.

Theorem 4.7.1. Let $D$ be a bounded open set and let $f$ be continuous on $\bar{D}$. Then $f$ is uniformly continuous on $\bar{D}$.

Proof. Suppose otherwise. Then there exists an $\varepsilon>0$ such that

$$
\forall n \exists r_{1}, r_{2} \in D\left(\left|r_{1}-r_{2}\right|<\frac{1}{n} \wedge\left|f\left(r_{1}\right)-f\left(r_{2}\right)\right| \geq \varepsilon\right) .
$$

By fixing an arithmetical enumeration of all complex rationals, we can arithmetically construct a sequence $\left(r_{11}, r_{12}\right),\left(r_{21}, r_{22}\right), \ldots$ in $D \times D$ such that

$$
\left|r_{n 1}-r_{n 2}\right|<\frac{1}{n} \wedge\left|f\left(r_{n 1}\right)-f\left(r_{n 2}\right)\right| \geq \varepsilon .
$$

By using the bisection method, we can construct an accumulation point $a$ of $r_{11}, r_{21}, r_{31}, \ldots$ as an abstract. Thus we have a contradiction.

The theorem of Weierstrass on the uniform convergence of analytic functions is proved in the usual manner in the following formulation.

Theorem 4.7.2. Let $\Omega_{0} \subseteq \Omega_{1} \subseteq \Omega_{2} \subseteq \cdots$ be regions and let $\Omega=\bigcup_{n} \Omega_{n}$. Suppose that $f_{n}(z)$ is analytic in $\Omega_{n}$, and that the sequence $\left\{f_{n}(z)\right\}$ converges to a limit function in $\Omega$, uniformly on every rectangle which is in some $\Omega_{m}$. Then $f(z)$ is analytic in $\Omega$. Moreover, $f_{n}^{\prime}(z)$ converges uniformly to $f^{\prime}(z)$ on every rectangle which is in some $\Omega_{m}$.

Since we gave the definition of $\bar{D}$ without giving the definition of a closed set, let us give it now.

Definition 4.7.1. A set of complex numbers is said to be closed, if its compliment is open.

The following two propositions are proved in the usual manner.

Proposition 4.7.3. If $F$ is closed, $\forall n\left(z_{n} \in F\right)$, and $\lim _{n \rightarrow \infty} z_{n}=z$, then $z \in F$.

Proposition 4.7.4. If $D$ is open, then $\bar{D}$ is closed.

Many theorems on series can be proved in our system without any change, e.g. the theorem on the Laurent series. 


\subsection{Final remarks}

The complex analysis so far developed will be called elementary complex analysis. If we are only concerned with limits, differentiation, integration, and applications of Cauchy's theorem, then the elementary complex analysis is sufficient to carry out all these proof. However we should remark on the limitations of elementary complex analysis.

1. Essential use of the axiom choice cannot be handled in elementary complex analysis. Thus the usual proofs of the theorem on normal families and the Riemann mapping theorem cannot be carried out in elementary complex analysis. If we need the theorem on the normal families, we have to prove it using special properties of each individual problem.

2. Many general topological considerations cannot be carried out in elementary complex analysis. For example, the usual proof of the following theorem in a general form cannot be carried out in the elementary complex analysis.

A topologically connected open set is arcwise connected. Therefore if we are interested in formalizing topological considerations of mathematical practice, we have to choose appropriate definitions of topological notions as we have done in the definition of arc, differentiable arc, region etc. Which specific definition we choose is usually not determined by specific informal arguments, for example, such arguments usually (i) only refer to a concept, not one of 'its' definitions, yet (ii) different definitions may be equivalent only for a stronger theory: the stronger the theory, the more definitions or properties are equivalent. For the specific case above, any of the usual definitions of a topologically connected open set in our mathematical practice implies in an elementary way that the set is arcwise connected, and so the theorem above becomes trivial.

3. The general theory of analytic extension and Riemann surfaces cannot be carried out in elementary complex analysis. Therefore we also have to make special treatments for special cases.

However the definition of Riemann's $\zeta$-function by analytic continuation can obviously be carried out in elementary complex analysis.

Thus classical analytic number theory, for example, the theory in [2] can be carried out in elementary complex analysis. 


\section{Bibliography}

[1] L. V. Ahlfors: Complex analysis, McGraw-Hill, New York, 1973.

[2] A. E. Ingham: The distribution of prime numbers, Hafner, New York, 1971.

[3] G. Kreisel: Some elementary inequalities, Koninkl. Nederl. Akad. van Wetenschappen, A, 55(1952), pp. 334-338.

[4] G. Kreisel: On the interpretation of non-finitiest proofs, I and II, Journal of Symbolic Logic, vol. 16(1951), pp. 214-267 and vol. 17(1952), pp. 43-58.

[5] G. Kreisel: Mathematical significance of consistency proofs, Journal of Symbolic Logic, vol. 23(1958), pp. 155-182, especially pp. 168-172.

[6] G. Kreisel: A survey of proof theory, Journal of Symbolic Logic 33 (1968), pp. 327-328, 361-362.

[7] G. Kreisel: Hilbert's programme and the search for automatic proof Procedures, in: Symposium on automatic demonstration, Springer Lecture Notes in Mathematics, 125(1970), pp. 128-146.

[8] G. Kreisel: Functions, ordinals, species, pp. 145-159, Logic, methodology and philosophy of science, Amsterdam, 1968.

[9] G. Kreisel : Analysis of the Cantor-Bendixson theorem by means of the analytic hierarchy, Bulletin of the Polish Academy of Science, vol. 7(1959), pp. $621-626$.

[10] G. Kreisel: Prédicativité, Bulletin Soc. Math. France, vol. 88(1960), pp. 371-391.

[11] G. Kreisel: The axiom of choice and the class of hyper-arithmetic functions, Dutch Academy A, vol. 65(1962), pp. 307-319.

[12] G. Kreisel: Applications of mathematical logic to various branches of mathematics, Colloque de la logique mathematique, August 1952, Paris (1954), pp. 37-49. 
[13] G. Kreisel: Ensembles récursivement mesurables et ensembles récursivement ouverts et fermés, (with $\mathrm{D}$. Lacombe), Comptes Rendus des Sciences de l'Académie des Sciences, vol. 245(1957), pp. 1106-1109.

[14] G. Kreisel: Axiomatizations of Non-Standard Analysis which are conservative extensions of formal systems for classical Standard Analysis pp. 93-106, in : Applications of Model Theory to Algebra, Analysis and Probability, New York(1969).

[15] G. Kreisel: Category theory and the foundations of mathematics, pp. 233245, in: Reports of the Midwest Category Seminar III, ed. S. McLane, Springer Lecture Notes in Mathematics 106(1969).

[16] G. Kreisel: Some uses of metamathematics, British Journal for the Philosophy of Science, vol. 7(1956), pp. 161-173.

[17] G. Kriesel: What have we learnt from Hilbert's second problem ? Proc. Symp. Pure Math. 28(1975): Mathematical developments arising from Hilbert's problems.

[18] G. Kreisel: A notion of mechanistic theory, Synthese 29(1974), 11-26.

[19] G. Kreisel: Formal rules and questions of justifying mathematical practice: Festschrift for P. Lorenzen's 60th birthday.

[20] P. Lorenzen: Differential and Integral, Akademische verlagsgesellschaft, Frankfurt am Main, 1965.

[21] H. V. Mangoldt and K. Knopp, Einführung in die höhere Mathematik, vol. 2, S. Hirzel Verlag, Stuttgart, 1968.

[22] J. C. Shepherdson : Non-standard models for fragments of number theory, The Theory of Models, North Holland, Amsterdam, 1970, pp. 342-358.

[23] G. Takeuti : Proof theory, North Holland, Amsterdam, 1975.

[24] H. Weyl: Das Kontinuum, De Gruyter, Leipzig, 1918. 


\section{Index}

$\neg, 7$
$\Rightarrow, 9$
$\sqrt{ }, 9$
$<, 61$
$\vee, 7$
$\wedge, 7$
${ }^{*} A, 87$

abstracts, 73

arc, 112

arithmetical, 74

B-function, 52

Baire function, 56

Boolean algebra of projections, 7

Boolean valued model $V^{(\mathcal{B})}$ of set theory, 8

commutable, 7

conservative extension, 70

Cut elimination Theorem, 77

$\mathcal{D}, 8$

EQ, 77

equality axiom, 77

FA, 83

$\mathrm{FA}_{1}^{\prime}, 79$

finite number of points in $\Omega, 116$

finite types, 73

formula, 73

in $(\mathcal{B}), 7$

in the strong sense, 33

inference, 75

LN, 79
LS, 76

mathematical induction, 77, 79

Maximum Principle, 14

measure algebras, 47

MI, 77

MI', 79

normal, 8

$(\omega, \omega)$-WDL, 36

$(\omega, \omega)$-weak distributive law, 36

$\left(\omega_{\alpha}, \omega_{\beta}\right)$-WDL, 36

orthomodular lattice, 43

our first system $\mathrm{FA}_{1}, 77$

$\mathrm{P}(a, b, A), 96$

partition of $[a, b], 96$

partition of unity, 12

piecewise convergence, 37

piecewise differentuable arc, 112

projection, 7

pseudo-Baire function, 56

pseudo-Borel, 56

pseudo-continuous, 56

pseudo-dense, 61

pseudo-open, 61

$\mathbb{R}^{(\mathcal{B})}, 15$

$\mathcal{R}, 7$

region, 114

regular semi-group, 34

replace all higher type free variables by $0=0,78$

resolution of the identity in $(\mathcal{B}), 16$

simply connected, 120

strongly measurable, 52 
"substitution", 74

system $T, 105$

system of simultaneous spectrums, 39

terms, 73

the height, 74

"types", 73

$z_{1} \square z_{2}, 113$ 